\title{
Three-dimensional Ultrasound in the Visualization of Fetal Anatomy in the Three Trimesters of Pregnancy
}

\author{
${ }^{1}$ Giovanni Centini, ${ }^{2}$ Lucia Rosignoli
}

\begin{abstract}
It is now widely considered that three- and four-dimensional (3-4D), namely 3D with a fourth dimension, time, offer too many possibilities to be ignored. Acquisition of a volume or region of interest is the great novelty of $3 \mathrm{D}$ technique. And just as exciting is the possibility of studying it in movement during ultrasound examination or afterward; also by other operators in an infinite number of section planes that can all be perfectly reproduced.
\end{abstract}

This paper aims to provide pictorial documentation of pregnancy monitoring with 3D images, sometimes with the corresponding $2 \mathrm{D}$ image, so readers can begin to habituate themselves and hopefully acquire a different and personal key to the 3D image.

Keywords: HDlive, Silhouette, Three-dimensional ultrasound, Visualization of fetal anatomy,

How to cite this article: Centini G, Rosignoli L. Threedimensional Ultrasound in the Visualization of Fetal Anatomy in the Three Trimesters of Pregnancy. Donald School J Ultrasound Obstet Gynecol 2018;12(3):197-248.

Source of support: Nil

Conflict of interest: None

\section{BIOPHYSICAL MONITORING OF PREGNANCY}

\section{An Embryo-fetal Anatomical Study by 3-4D Ultrasound}

During the 1970s, the use of ultrasound in obstetric diagnostics was a driving force in the study of the fetus, revolutionizing the concept of prenatal monitoring. The development of increasingly sophisticated techniques such as high frequency, real-time, echo-Doppler flow imaging, color and power Doppler and the second harmonic, was followed in the 90's by a period of relative immobility for ultrasound up-grading. However, since the turn of the millennium, volumetric probes that store volume samples, acquiring up to 25 images per second (and multiples of this figure are already in view), have transformed the classical concept of two-dimensional ultrasound, generating enormous interest and a great

\footnotetext{
${ }^{1}$ President, ${ }^{2}$ Prenatal Diagnosis

${ }^{1}$ Nannini Foundation for Maternità and Childhood, Siena, Italy

${ }^{2}$ Meyer Hospital, Florence, Italy

Corresponding Author: Giovanni Centini, President, Nannin Foundation for Maternità and Childhood, Siena, Italy, e-mail: giovannicentini@virgilio.it
}

spurt of research which still has to be translated into scientific knowledge. So it is now widely considered that three- and four-dimensional (3-4D), namely 3D with a fourth dimension, time, offer too many possibilities to be ignored. ${ }^{1-3}$ Acquisition of a volume or region of interest is the great novelty of 3D technique. And just as exciting is the possibility of studying it in movement during ultrasound examination or afterward; also by other operators in an infinite number of section planes that can all be perfectly reproduced.

Moreover, the many systems of representing acquired volumes make this technique very similar to computed axial tomography (CAT) and nuclear magnetic resonance (NMR) imaging:

- Multiplanar scan: The image can be visualized and studied in the three classical scan planes: coronal, sagittal and transverse;

- Minimum rendering: This is the classical 3D image of external embryofetal or other anatomical morphology;

- Maximum rendering: This highlights deep echoes to visualize skeletal details;

- Glass body: This highlights blood vessels in an anatomical part which is rendered transparent like glass;

- Vocal: Enabling volumes to be calculated with great accuracy;

- Invert: The transformation of liquid into solid parts;

- $\quad$ STIC (spatiotemporal imaging correlation): Storage of a moving volume over a time interval with the possibility of representing and studying it later in slow motion and in different planes;

- TUI (tomography ultrasound imaging) or multislice: A field is sectioned up to 27 scans at predefined distances and in real time (like NMR);

- 4D:3D represented in time;

- VCI-c-plane: Improved tissue contrast resolution in real time (4D), coronal plane imaging (orthogonal plane of the scan plane);

- B-flow: The direct visualization of blood reflectorshas made B-mode flow imaging (B-flow) possible without the limitations of Doppler technology, angle independent;

- Omni-view: To study the same volume by VCI-cplane multiple sections. It is possible to shape the box of observation (linear, curve, etc .) to the region of interest (ROI). This possibility is significant because the human anatomy usually has a curve or round shape. By omni-view we can realize pictures more detailed; ${ }^{4}$ 
- Sono automated volume calculation: Sono automated volume calculation (SonoAVC) the possibility to detect areas by different color and to calculate the volume of these areas;

- High-definition live source: High-definition live source or real-time US (Hdlive), a new ultrasound software, combines a movable virtual adjustable light source in software that calculates the proportion of light reflecting through surface structures, depending on light direction. The light source can be manually positioned to illuminate the desired area of interest. The ultrasound technician can control light intensity to create shadows that enhance image quality. Hdlive is an innovation that will render even more realistic images of fetal anatomy and gynaecologic lesions. ${ }^{5}$ Three-dimensional Hdlive further "humanizes" the fetus, enables detailed observation of the fetal face in the first trimester. Hdlive is one the most promising forms of non-invasive diagnostic and embryological phenomena, once matters for textbooks are now routinely recorded with outstanding clarity. New advances deserve the adjective "breathtaking," including a 4D parallel study of the structural and functional early human development. 6,7

- Silhouette or transparency, a new advanced tool of Hdlive to evidence the shape of the embryo-fetal body and internal cavity of brain and body. The silhouette is a good ultrasound tool to study the embryo-fetal head in the first trimester ${ }^{8}$ (Figs 1 to 12).

Other possibilities of use are the electronic scalpel which eliminates parts, not of interest and rotat es about orthogonal axes.

Today, ultrasound research is so active that new applications have probably been found as these words are being written. One new advance is already available, the electronic matrix probe. The possibilities of electronic rather than volumetric probes, which can combine electronic scans with mechanical movement to cover the area of interest, will provide even more sophisticated images. And the possibility of obtaining volume samples from unthinkable angles and perspectives will be especially useful in cardiology. ${ }^{9}$ These innovations, however, require relatively long development periods before they are applied.

The above discourse raises the question whether 3-4D offers greater certainty in embryo study and detection of fetal and ovarian pathology. ${ }^{10-12}$ The scientific community seems unanimous in considering that we cannot yet fully answer this question, but ten years after the finalization of the volumetric probe were acquired many experiences that can make a real contribution to define the role and importance of 3-4D in the study of various embryo- fetal's organs and systems.

However, there are already many papers comparing $2 \mathrm{D}$ and $3 \mathrm{D}$, and their results define the use of 3-4D complementary to $2 \mathrm{D}$, with the exception for the neurosonology and fetal cardiology, where can offer the better possibility to explore physiological and pathological fetal anatomy. This technique has unique applications, but it is commonly considered that 3-4D offers an improvement and completion of $2 \mathrm{D}$, of such interest that it cannot be forgone once tried. Hence there are specific situations in which $3 \mathrm{D}$ is indispensable, such as when a coronal scan is needed (visualization of the corpus callosum or a fetal profile in anterior occipital position) through a good instrument in the hands of an experienced sonographer can meet all the needs of ultrasound monitoring in pregnancy. Those with long experience with 2D, and subsequently with 3-4D, know the pleasure of working better and obtaining images superior to those obtained by 2D. The possibility of saving a volume and studying it later, discussing it with other operators and visualising it in an infinite number of planes, superior to those obtained with $2 \mathrm{D}$ and perfectly reproducible, is

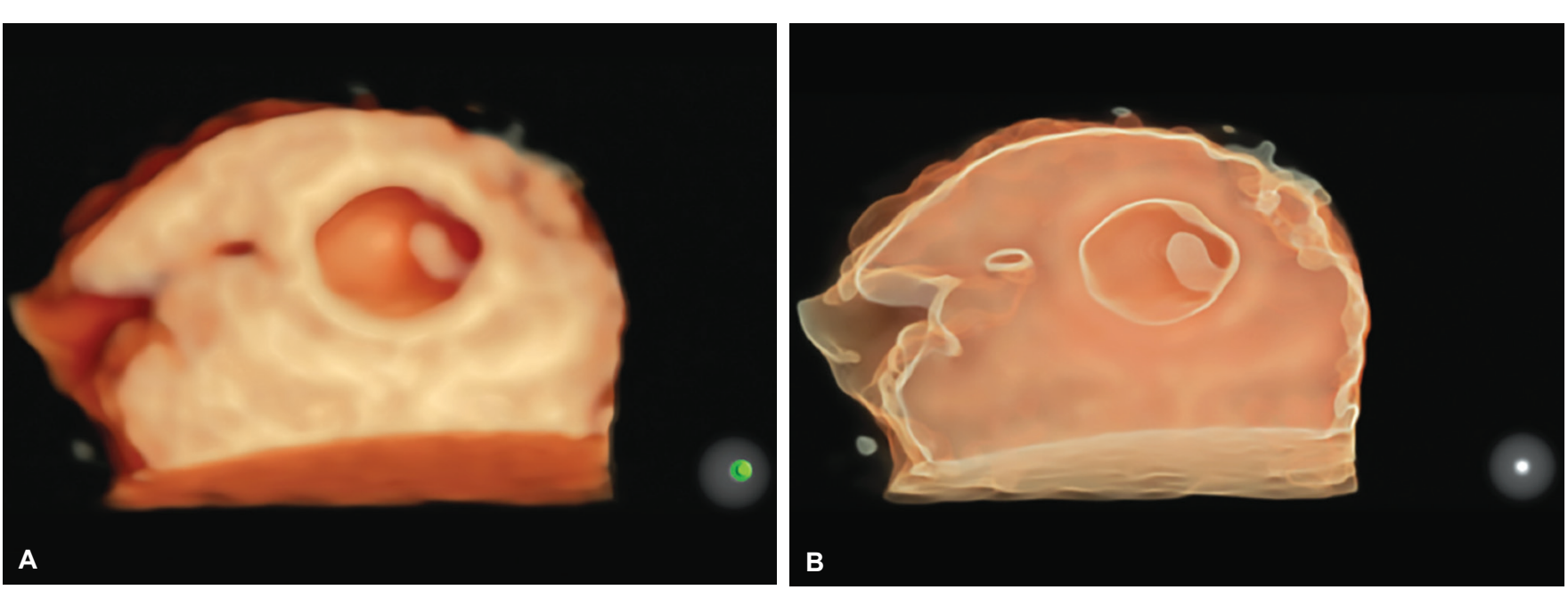

Figs 1A and B: Weeks $5+5 \mathrm{~d}$ : (A) HDlive of gestational sac and embryo with front light; (B) The same picture with silhouette. The shape of embryo's cerebral ventricles are highlighted 

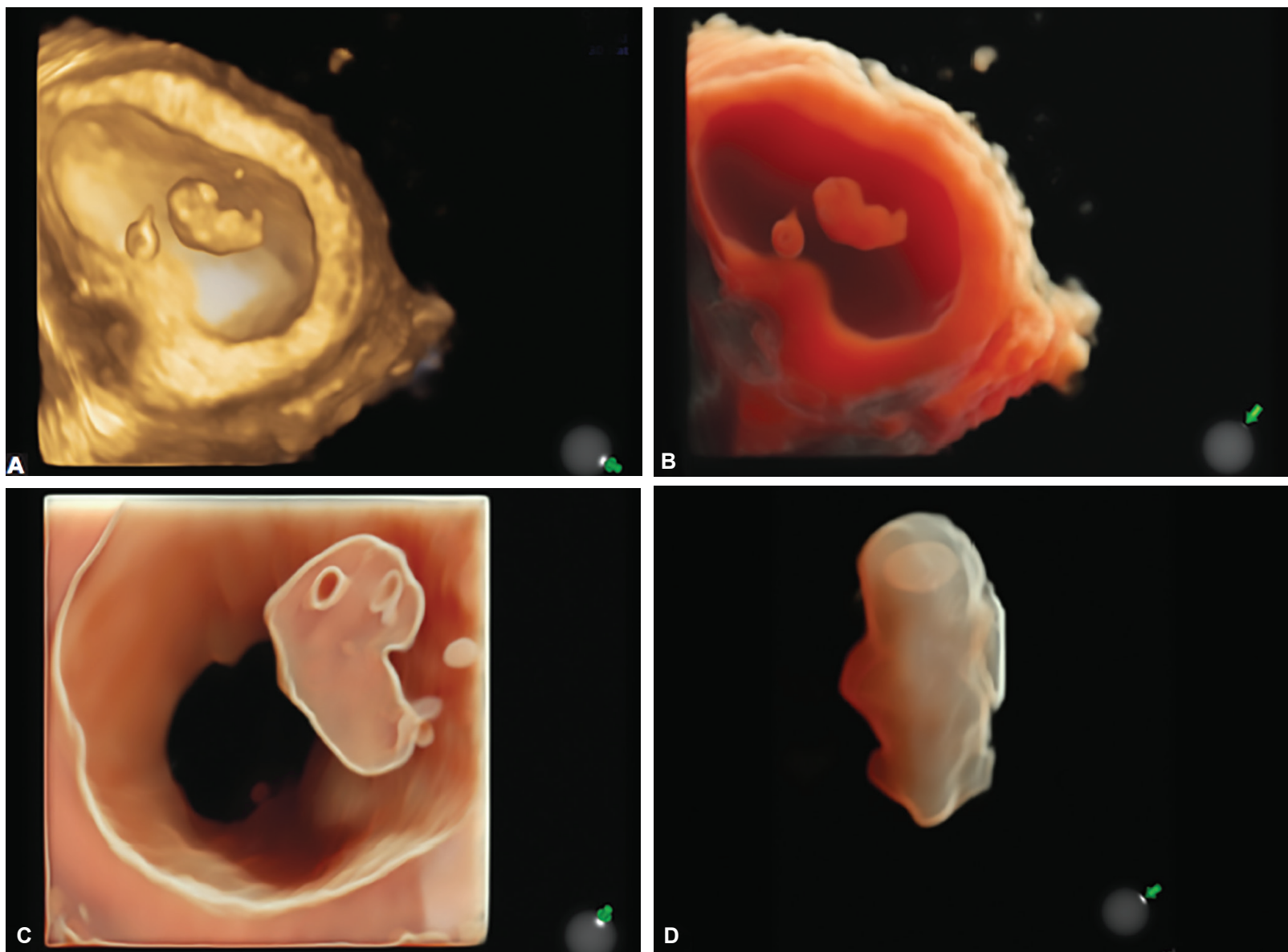

Figs 2A to D: Weeks $8+4 \mathrm{~d}$ : (A) HDlive with lateral light; (B) With behind light; (C) Silhouette to evidence the cerebral ventricles;

(D) Silhouette, retrovision of embryo with lateral light; the evidence of romboencephalon
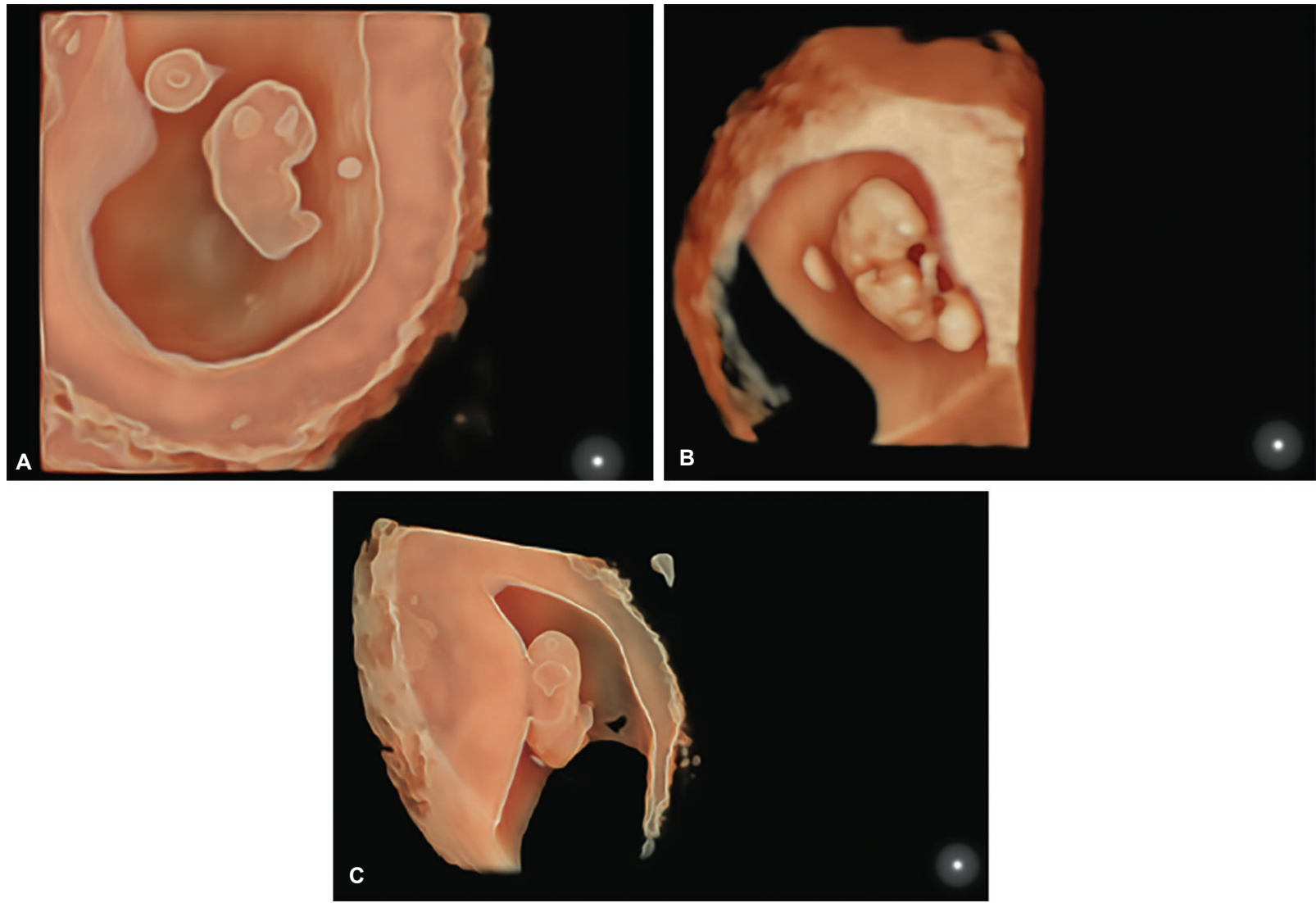

Figs 3 A to C: Weeks $8+5 \mathrm{~d}$ : (A) HDlive with lateral light to see cerebral ventricles and yolk sac's cavity; (B) HDlive; (C) Silhouette with frontal light; by posterior vision of embryo, it is possible evidence the prosencephalon and rhombencephalon 

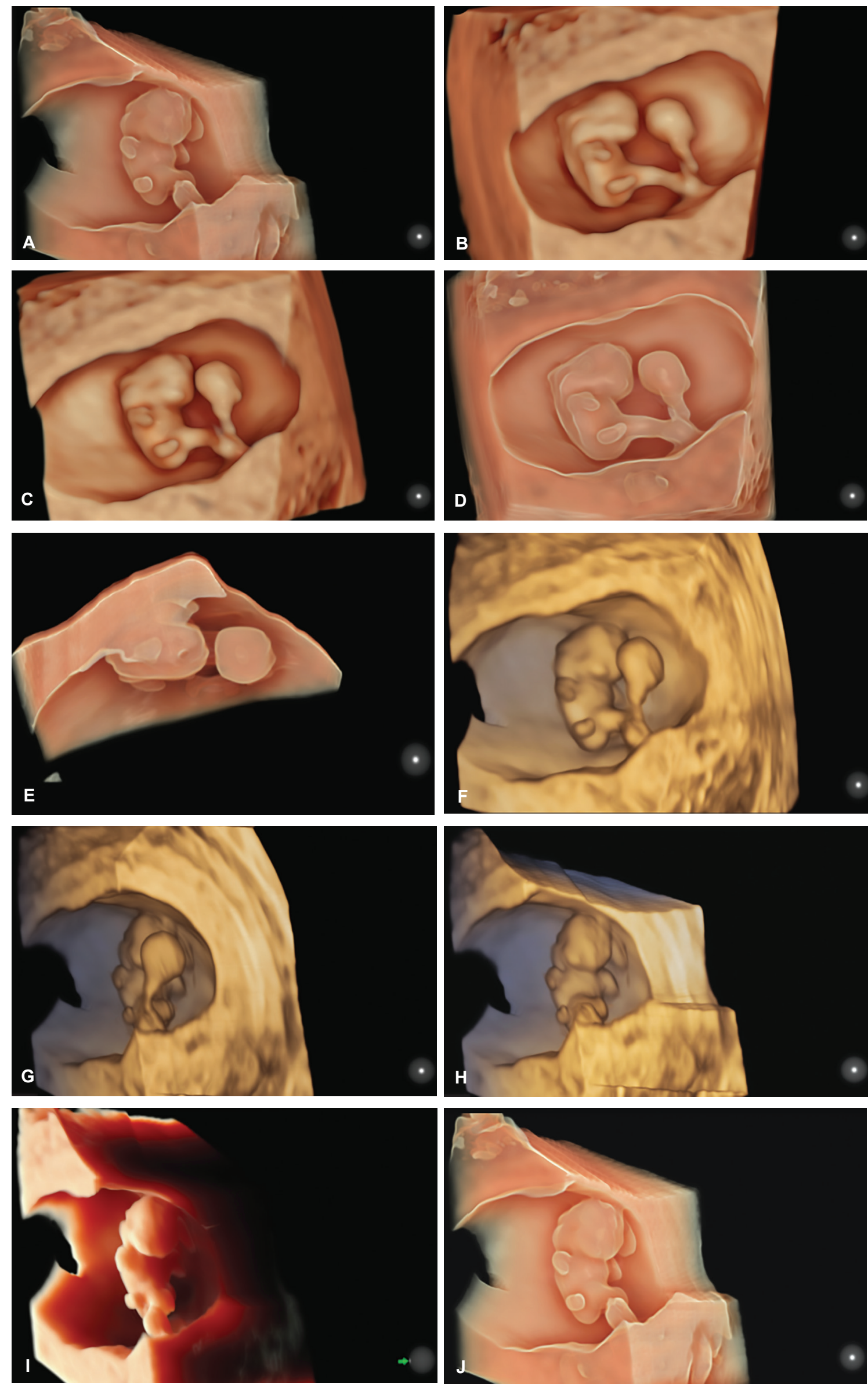

Figs 4A to J: Weeks $8+6 \mathrm{~d}$ : A different point of view of the embryo on HDlive and silhouette 

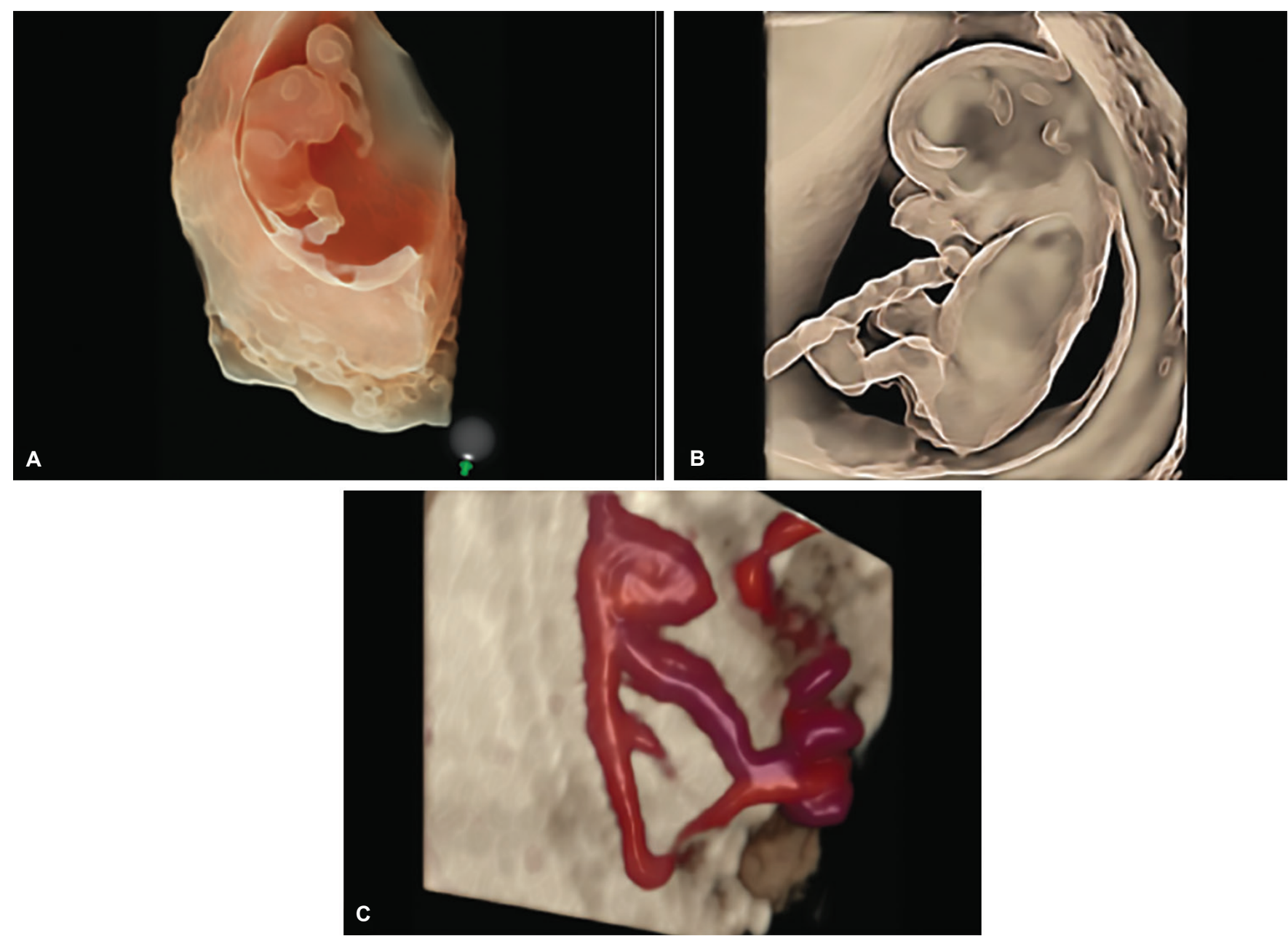

Figs 5A to C: Weeks $10+0 \mathrm{~d}:(\mathrm{A}$ and $\mathrm{B})$ Silhouette of the embryo; (C) HDlive of vascular circulation

as close as one can imagine to CAT or NMR scans, with the advantage of speed, easy repetition and much lower costs. The old recorded cassette to transmit images of a malformation, for example, cannot compare with the volumetric acquisition. It seems inevitable that in the near future nearly all instruments will be equipped with 3-4D, even if not all sonographers know how to exploit it fully. It is, therefore, necessary to begin to train experts who think and work directly in 3D without having to make the often difficult transition from 2D. Sonographers of tomorrow (today) will undoubtedly obtain better results than those who began with $2 \mathrm{D}$ because they will already have in mind the field of interest to explore in 3D.

This paper aims to provide pictorial documentation of pregnancy monitoring with 3D images, sometimes with the corresponding 2D image, so readers can begin to habituate themselves and hopefully acquire a different and personal key to the 3D image.

\section{FIRST TRIMESTER OF PREGNANCY}

\section{From Conception to Week 10}

The sophistication achieved by ultrasound instruments associated with clinical and ultrasound knowledge and know-how unthinkable only ten years ago enables us to monitor pregnancy from before conception. For fertility control, 2D ultrasound with power or color Doppler makes it possible to determine with sufficient certainty the following aspects on day: ${ }^{12}$

- Uterine morphology and myometrial structure; the tridimensional scan in the coronal section is a useful instrument comparable to ISG-RNM to explore the uterine cavity and find the anatomical uterine malformation which represents 3 to $5 \%$ : arcuate uterus, bidelfus and bicornuate;

- Endometrial echostructure and morphology by detecting a three-line image characteristic of the ovulatory period and myometrial vascularisation; 


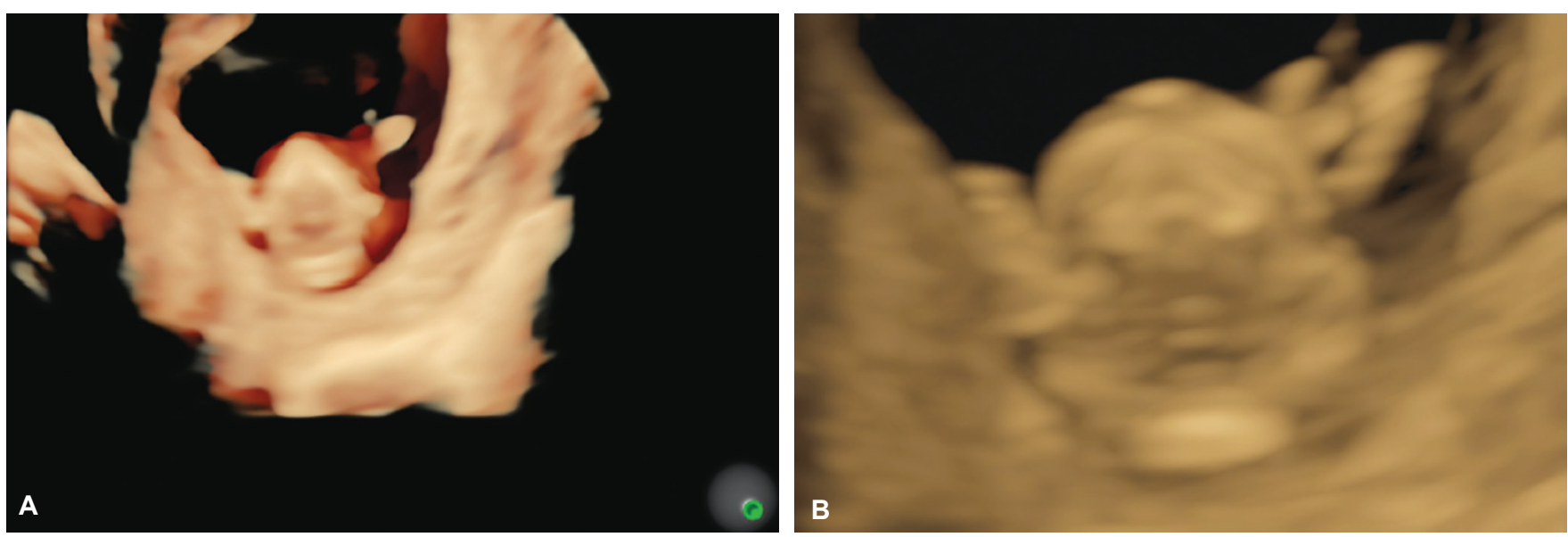

Figs 6 A and B: Weeks $12+0 \mathrm{~d}$ : The study of secondary palate (delta sign) on HDlive rendering and bone
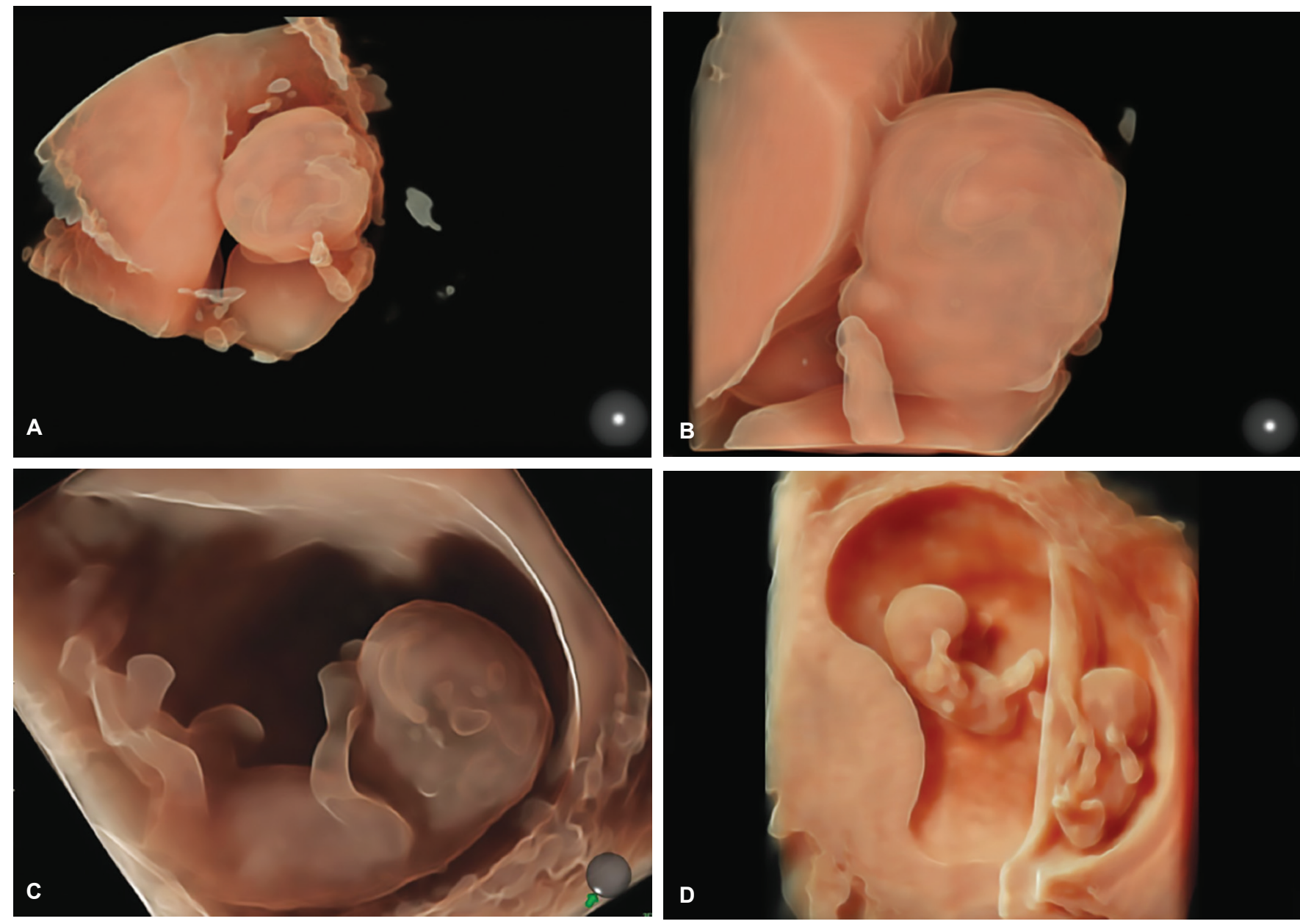

Figs 7A to D: Weeks $12+0$ d: (A to C) Silhouette: the vision of right ventricles; (D) Silhouette of twin

- Uterine artery flow values which should not have a pulsatility index of less than 3;

- The dominant follicle measuring 16 to $18 \mathrm{~mm}$ with peak systolic velocity (PSV) of 5 to $10 \mathrm{~cm} / \mathrm{sec}$ in neighbouring vessels;

- Four to five antral follicles per ovary with PSV of 6 to $12 \mathrm{~cm} / \mathrm{sec}$ in stromal vessels (Figs 13 to 15).

By means of 3-4D or better the 4D VCI-c-plane system it is possible to obtain a correct view of the uterine cavity $^{13,14}$ (Fig. 14). This means that with only one examination, the existence of anatomical-functional conditions for pregnancy can be ascertained. Once conception has occurred, ultrasound monitoring of pregnancy should not necessarily begin in the first weeks of gestation. This type of protocol is usually used for medically assisted conception, however it is possible to follow the progress of pregnancy week by week, acquiring important information on physiological or pathological evolution by monitoring embryo-foetal growth and studying embryo-foetal anatomy. At the present 

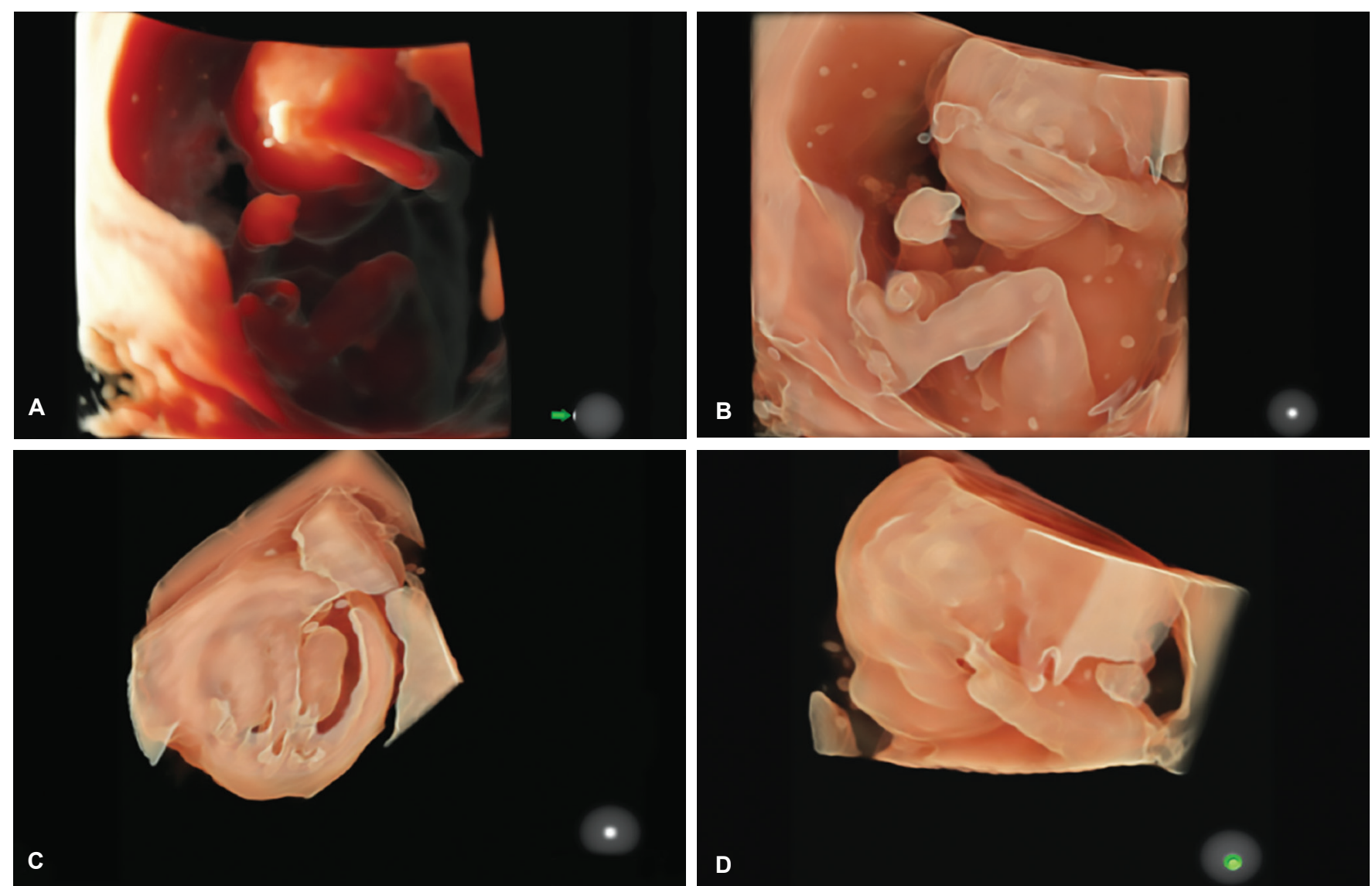

Figs 8A to D: Weeks $14+0 \mathrm{~d}$ : The fetus by HDlive and by silhouette with different point of view light

time, 3-4D volumetric acquisition provides superior images in terms of definition and visual impact, but only slight improvements in diagnostic capacity with respect to $2 \mathrm{D}$.

\section{An Ultrasound View of the First Trimester of Pregnancy}

In indicating the period of embryo-foetal development, it is correct to distinguish between menstrual age and true gestational age. Menstrual age is counted from the last menstrual period and is unreliable. About $40 \%$ of pregnant women, or one in two, have ovulation's problems for various reasons. Moreover, even in women with physiological ovulation's patterns, conception may occur between day 11 and day 17 of the menstrual cycle. It is therefore essential to date pregnancy by means of ultrasound parameters during the first trimester. Gestational age is counted from when the gametes fuse and is on average 14 days less than the menstrual age used by embryologists. ${ }^{15}$ Internationally, in fact, obstetricians now speak of gestational age when they really mean menstrual age. A compromise is the term ultrasound age, namely age established by ultrasound. When it is desired to indicate the effective period of embryo-foetal life, however, this is usually specified.
In order to better understand ultrasound images, especially for early detection of anatomical structures and embryo-foetal morphology, it is useful to refer to embryology, though naturally the anatomical age is earlier. In practice, it is important to be clear about the terms used.

The first trimester of pregnancy is divided into three periods: pre- embryonic, embryonic and foetal.

\section{Pre-embryonic Period}

Embryology: Days 4 to 19

Day 4-Morula

Days 4 to 7-Implant of avillous morula in the uterus

Days 8 to 12-Implant of blastocyst deep in decidua; presence of amniotic and celomatic cavities

Days 13 to 19-Presence of chorionic villi, yolk sac (YS) and neural plate.

\section{Ultrasonography: Weeks 3 and 4 of Gestation}

Ultrasound detects decidualisation of the endometrium and the luteal body but cannot confirm pregnancy. Endometrial flow can be assessed and when absent suggests lack of implantation (Fig. 16).

\section{Embryonic Period}

Embryology: Days 17 to 49 (seven full weeks)

Ultrasonography: Weeks 5 to 9 inclusive (up to day 63) 

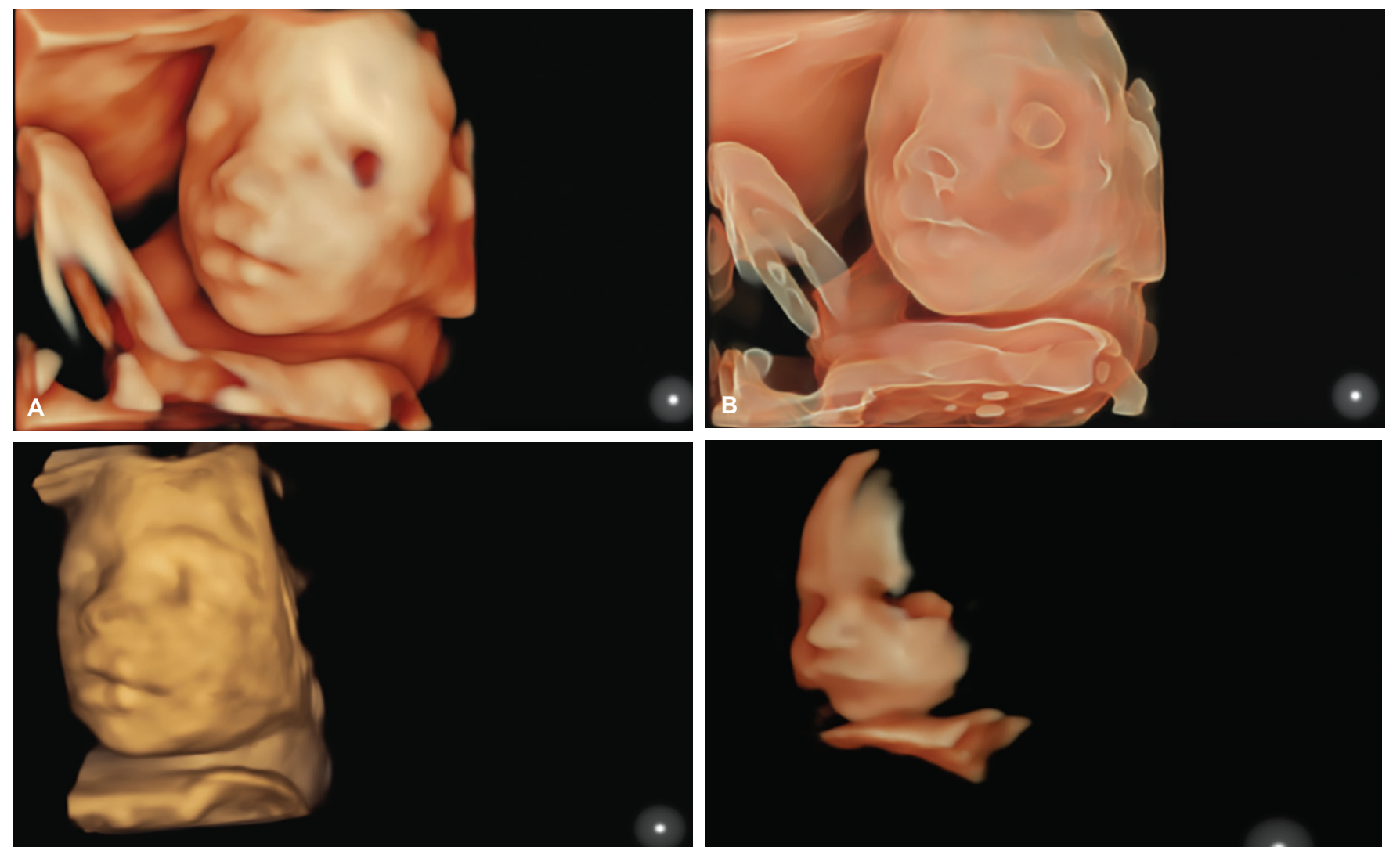

C
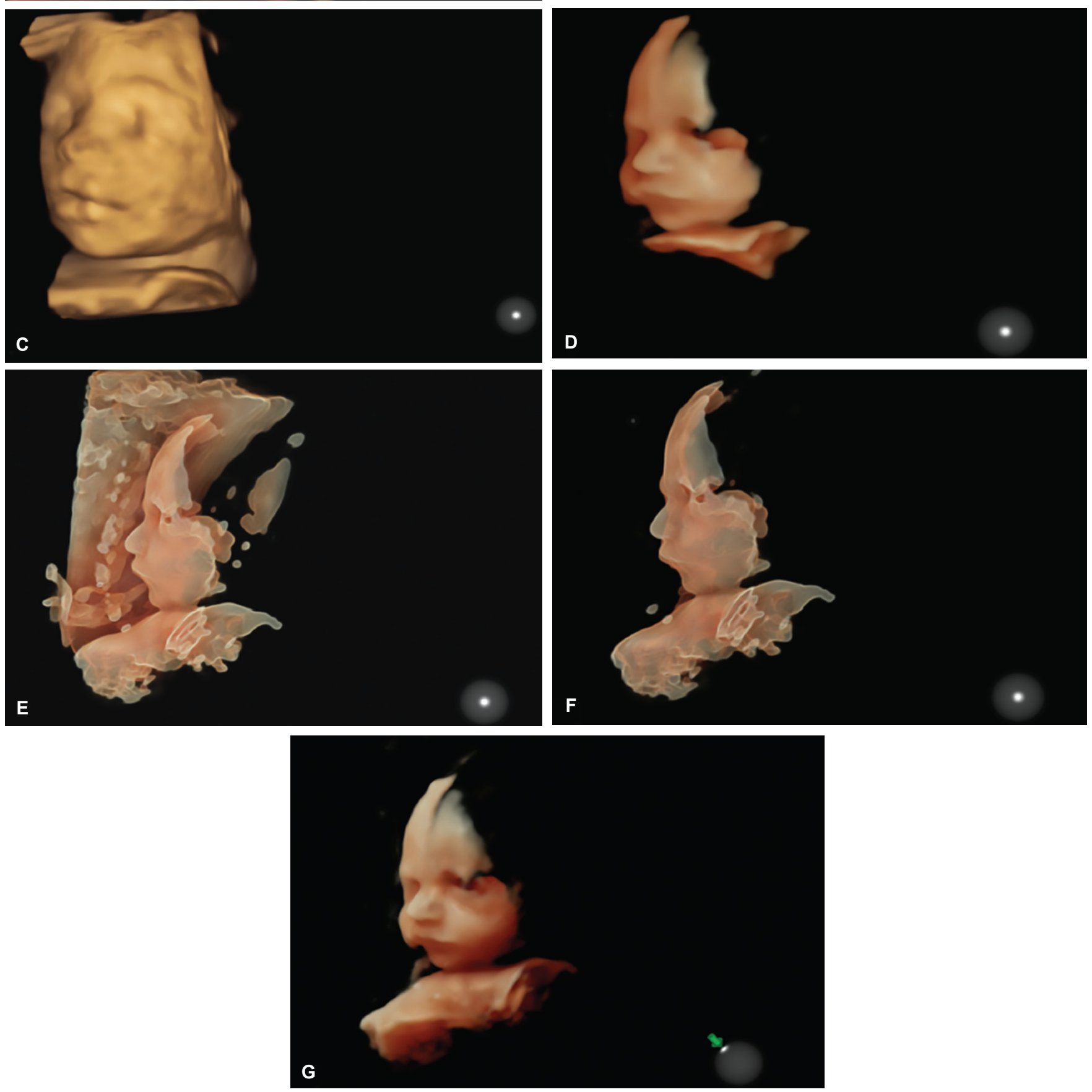

Figs 9A to G: Weeks $22+0$ d: "Humanization" of the face by HDlive and silhouette 

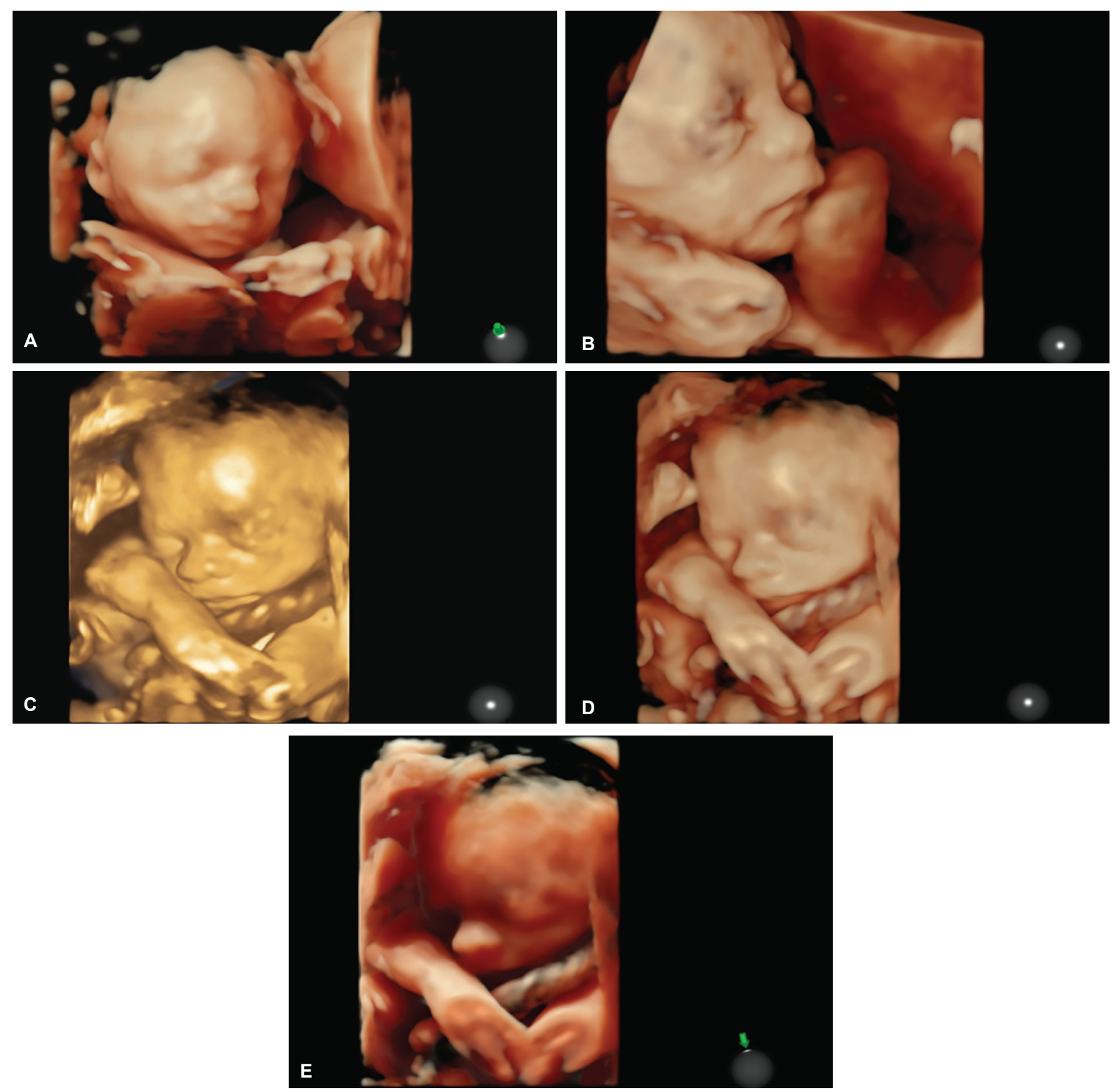

Figs $10 \mathrm{~A}$ to $\mathrm{E}$ : Weeks $23+0 \mathrm{~d}$ : Evidence of the umbilical cord by different use of the light on HDlive

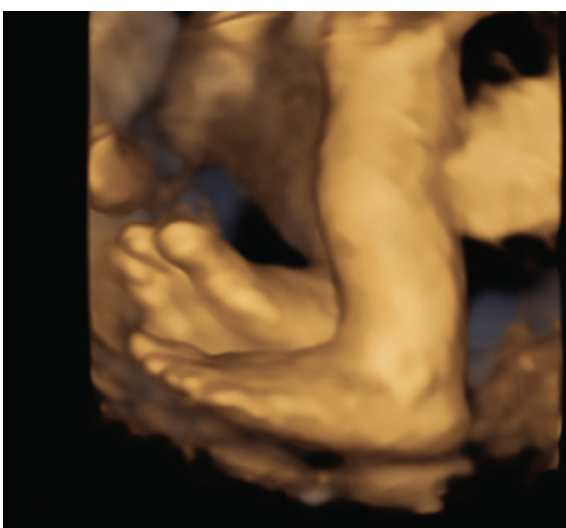

A

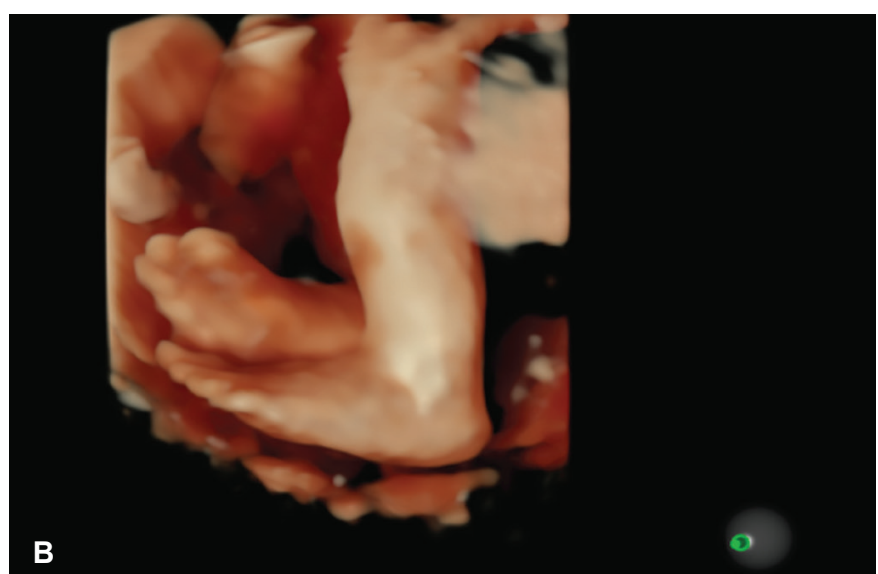

B

Figs 11A and B: Weeks $23+0$ d: Study of the feet by HDlive and silhouette 

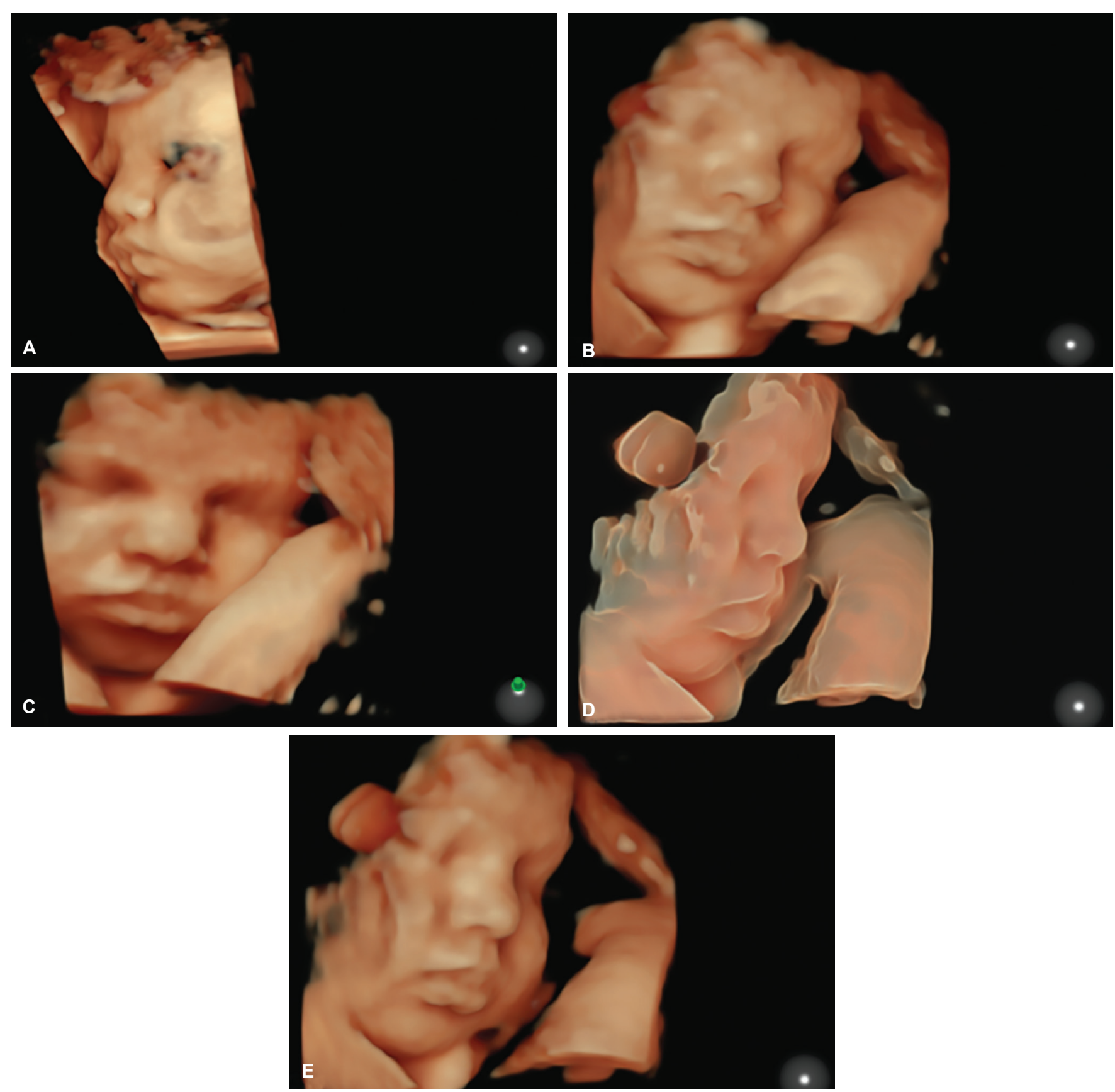

Figs $12 \mathrm{~A}$ to $\mathrm{E}:$ Weeks $37+0 \mathrm{~d}$ : Humanization of the face by HDlive and silhouette

Comparison of tissue, organ and system formation detected and studied anatomically during the embryonic period demonstrates the capacities and limits of ultrasound for monitoring the product of conception. Another small distinction between embryology and ultrasound is that in the former case reference is made to exact parameters such as crown rump length (CRL) or the presence of tissues, organs and systems, whereas in the latter gestational age is usually indicated in weeks (the definition of which we have already discussed), with considerable limitations.

Embryology: Days 20 to 23

$C R L=1.5$ to $2.0 \mathrm{~mm}$
Cloacal membranes and posterior cerebral vesicle (rhombencephalon).

\section{Week 5 (Ultrasonographic)}

From 4 weeks +0 days to 4 weeks +6 days from the last menstrual period

Week 5 of gestation: It is possible to identify the gestation chamber (GC) from 4 weeks +2 to 3 days in pregnancies with regular menstrual cycles, and by the end of week 5 this is possible in almost $100 \%$ of pregnancies. The number of GCs can also be determined. Spiral circulation around the GC can be detected from week 4; usingmultiplanar 3D with surface rendering it is easier to see 

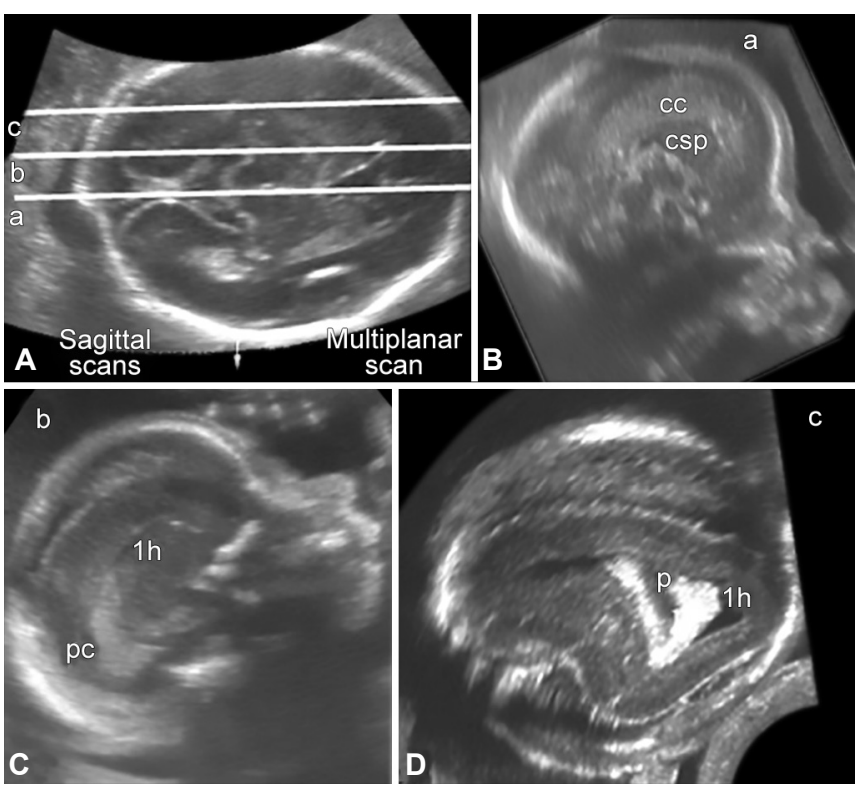

Figs 13A to D: Sagittal scans. Image a shows the corpus callosum and cavum septi pellucidi perfectly. This section is very difficult to obtain by 2D, but easy by 3D multiplanar scan. The parasagittal scan offers a good view of the lateral horn and the anterior, posterior and sub temporal horns

(Abbreviations: Cc, corpus callo sum; csp, cavum septi pellucidi; Ih, lateral horn; $p$, choroid plexus)
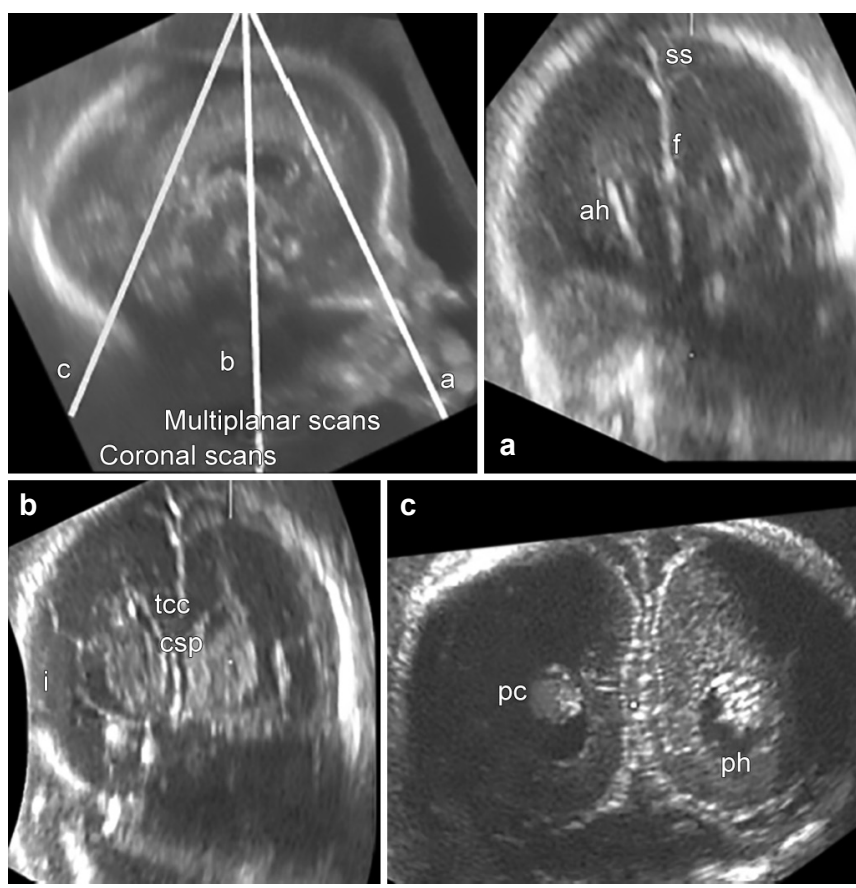

Fig. 14: Coronal scans. Section a is known as ox head because the anterior horns resemble those of an ox; Section b shows a good view of the corpus callosum, cavum septi pellucidi and lateral ventricles; Section c is known as owl eyes because of the contrast between the posterior horn and the white nervous system with eye-like choroid plexi

(Abbreviations: ah: anterior horn; csp, cavum septi pellucidi; $f$, falx; i, insula;lv, lateral ventricle; $\mathrm{cp}$, choroid plexus; ph, posterior horn)

the yolk sac (YS). Detection of the GC in uterus is an essential condition for excluding ectopic pregnancy and its eccentric position with respect to the uterine cavity, the presence of chorionic villi and consequent peripheral vascularisation, ${ }^{16}$ combined with hCG monitoring, leave progressively less room for diagnostic uncertainty. It is also possible to visualise vascularisation of the myometrium and decidua and check their homogeneous vasculogenesis, though these observation do not yet have clinical implications.

Detection of a GC measuring 2 to $4 \mathrm{~mm}$ in week 5 enables exact dating of pregnancy with an error of only 2 to 3 days. Visualisation of the YS may be possible in the same week and is the first ultrasound-detectable embryonic structure; 2 to 4 days later it is possible to see the embryo as a double bubble. In the following days and in practice from week 6 , the yolk sac moves away from the embryo and the amnios is seen to divide the celomatic from the amniotic cavity. The yolk sac appears as a transonic ring above the cephalic pole of the embryo, growing slowly until weeks 8 to 9 without exceeding a diameter of $5 \mathrm{~mm}$ and then reducing progressively to disappear between weeks 12 and 14. An absent yolk sac or a large, non spherical, hyperechogenic one with echo-rich internal structure is a condition associated with a poor prognosis for the pregnancy. A GC of mean diameter greater or equal to $20 \mathrm{~mm}$, of lower than expected volume for gestational age, bounded by a thin trophoblast towards the periphery and devoid of embryo suggests blighted ovum syndrome. In such cases it is advisable to repeat ultrasound examination a week later (for diagnosis at week 7).

\section{Embryology: Days 24 to 27}

$\mathrm{CRL}=4.0 \mathrm{~mm}$, head and body distinguishable;

- Formation of prosencephalic cerebral vesicles or anterior brain, mesencephalon or middle brain and rhombencephalon or posterior brain, which subsequently give rise to:

- Prosencephalon

- Median vesicle (diencephalon) that gives rise to third ventricle

- Two lateral vesicles (telencephalon) that give rise to the hemispheres and lateral ventricles;

- Formation of optical vesicles;

- Formation of falxcerebri;

- Formation of limbs, liver, pancreas, lungs, thyroid and mesonephrium tubes;

- Fusion of two cardiac tubes along the median line and initiation of heart activity (Fig. 17).

\section{Week 6 (Ultrasonographic)}

\section{From 5 weeks +0 days to 5 weeks +6 days}

The GC should still be visible; if not, hCG should be assayed and repeated a week later to determine the possibility of delayed conception or ectopic pregnancy. Bi-, tri-, chorionic multiple pregnancy is readily detected. The 

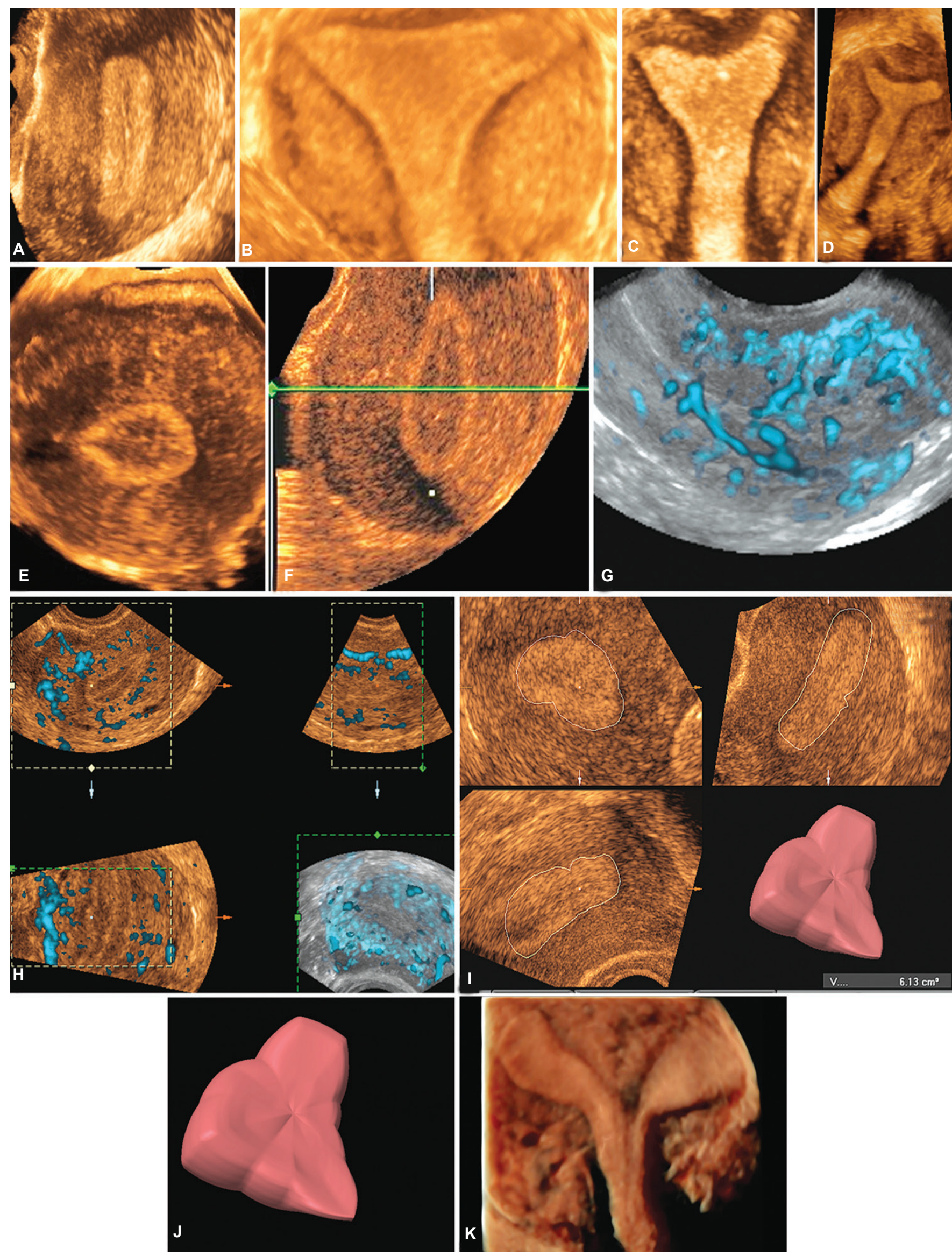

Figs 15A to K: Different kinds of physiological endometrium at 12 days, a zoom of the three lines and myometrial vascularization and volume by VOCAL. (A) Multiplanar; (B to D) Surface rendering normal uterine shape; (E to H) Luteal phase; (I and J) Vocal; (K) HDlive of bicornuate uterus 
YS is detected in almost $100 \%$ of cases towards the end of the week and the double bubble image is increasingly frequent with a percentage detection of the embryo of about 20 to $40 \%$. CRL is 1.5 to $4.0 \mathrm{~mm}$ at the end of week 6 and embryo heart beat $(93-106 \mathrm{bpm})$ is visible. From now until week 12, CRL is the most reliable biometric value for dating pregnancy; mean error in expert hands is \pm 2 to 3 days.

Embryology: 28 to 35 days

$\mathrm{CRL}=6$ to $9 \mathrm{~mm}$;

slow heart beat evident through chest wall;

the hemispheres increase;

budding of limbs;

primitive intestine present;

first movements of embryo.(Fig.18)

\section{Week 7 (Ultrasonographic)}

From 6 weeks +0 days to 6 weeks +6 days

The amnios is still distinct from the chorion; the yolk sac is increasingly distant from the embryo, sometimes already compressed between the two membranes; prosencephalon and rhombencephalon detectable; budding of limbs; first movements of embryo.

Embryology: 36 to 42 days $\mathrm{CRL}=11$ to $20 \mathrm{~mm}$;
Formation of olfactory and auditory systems;

separation of aortic-pulmonary trunk and of right and left atrioventricular canals;

herniation of midgut in umbilical cord;

formation of limb extremities (fingers and toes); spine detectable (in more detail with 3D) (Fig. 19).

\section{Week 8 (Ultrasonographic)}

From 7 weeks +0 days to 7 weeks +6 days

The amnios is still distinct from the chorion; the two membranes constrain and envelop the YS, making it disappear when they fuse, which usually occurs at 14 to 16 weeks; choroid plexuses present; herniated intestine in umbilical cord which should resume its intraabdominal position by week 12 (omphalocele differentiation); embryo tachycardia > 110 bpm; evident movements of embryo; facial features detectable; extremities detectable. Embryology: 43 to 49 days

$\mathrm{CRL}=22-30 \mathrm{~mm}$;

Legs form circle with knees turned out and feet in contact (frog attitude);

Formation of eyebrows and external ear (Fig. 20).

\section{Week 9 (Ultrasonographic)}

From 8 weeks +0 days to 8 weeks +6 days
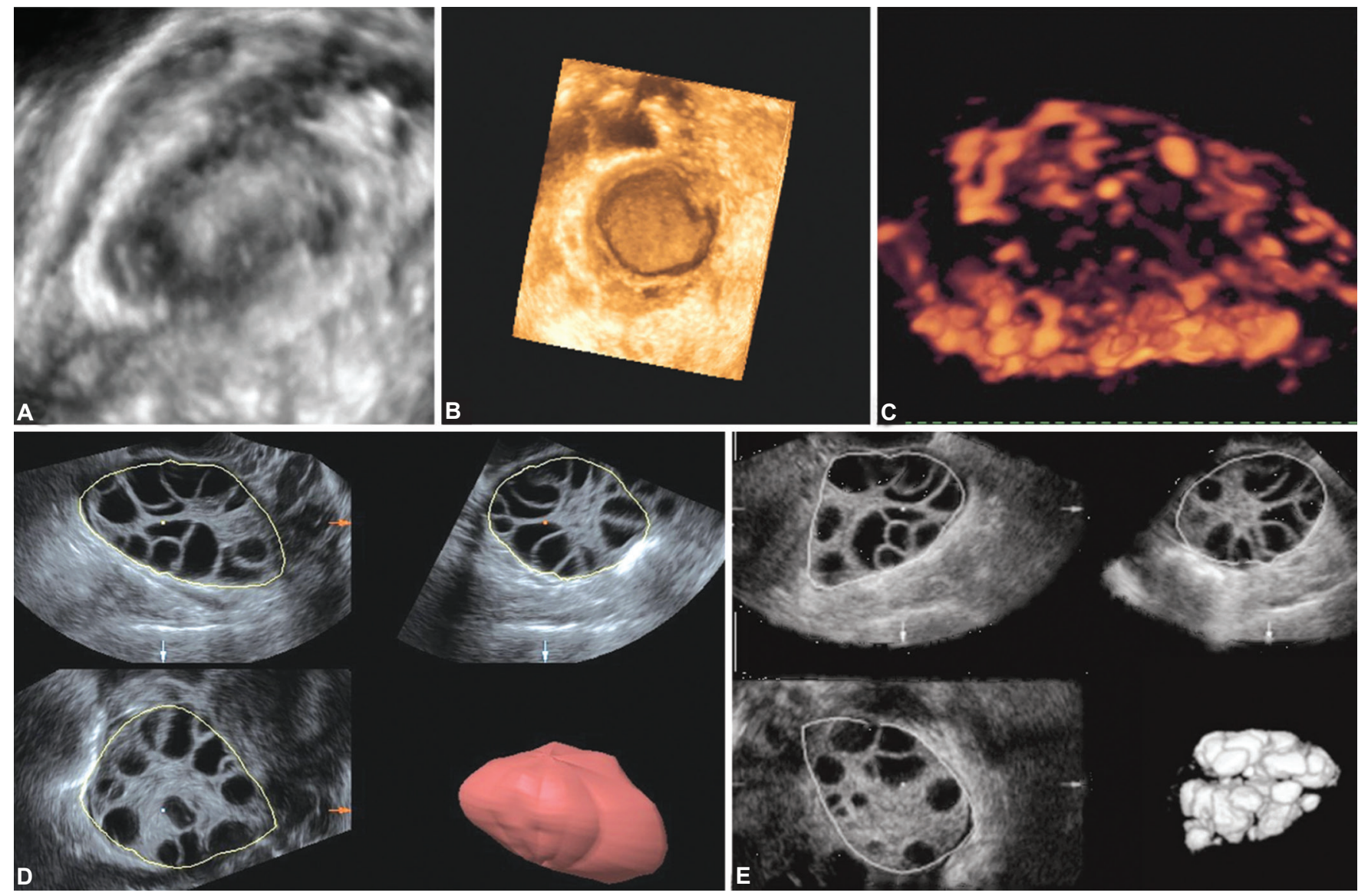

Figs 16A to E: The number of follicles is easier to determine by 3D in the first part of the cycle (preferably by Vocal and Invert during hyperstimulation) and the morphology and vascularization of the Graafian follicle are more realistic 

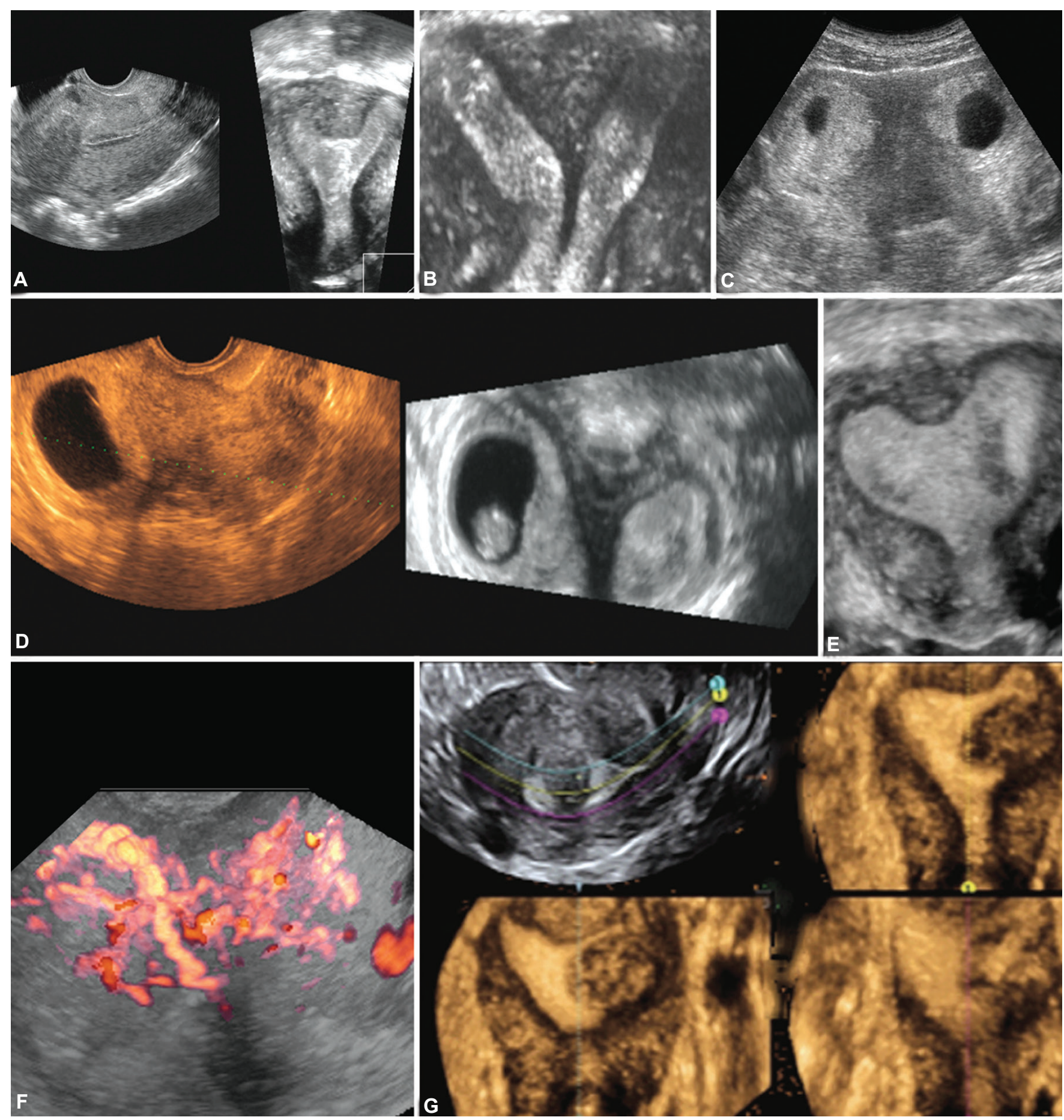

Figs 17A to G: 3-4D offers a good view of the endometrium and can enable diagnosis of different kinds of uterine malformation.

In early pregnancy (8th week) detection is easier, but for correct diagnosis it is important to see vascularization of two cornua. (A and B) Utero didelfo; (C and D) Bicornuate uterus with pregnancy at 6th and 8th week; ( $E$ and F) Didelfo uterus with vessels; (G) By multiplanar and surface it is easier to define the position of myoma

The embryo is clearly visible through vaginal and abdominal windows and begins to appear human. Its movements are often jumpy and abrupt. More articulated and refined movements are not seen until development of the neopallium in months 6 to 7 . The face is clearly delineated, especially by $3 \mathrm{D}$ volumetric scans. In the brain, the hemispheres and ventricles with their posterior (choroid plexuses) and anterior horns are observed developing from the two vesicles of the telencephalon. The brainstem is sometimes detectable. The rhombencephalon persists and is dividing to form the fourth ventricle (metencephalon) and the spinal cord (myelencephalon). The spine and ribs are clearly visible. Differentiation of chorion laeve and chorion frondosum.

The embryonic period comes to an end after week 9 , ushering in the fetal period with its rapid longitudinal (hyperplastic) growth that continues until week 20, with further differentiation and organisation of organs and tissues formed in the embryonic period and with acquisition of specific functions (Fig. 21). 

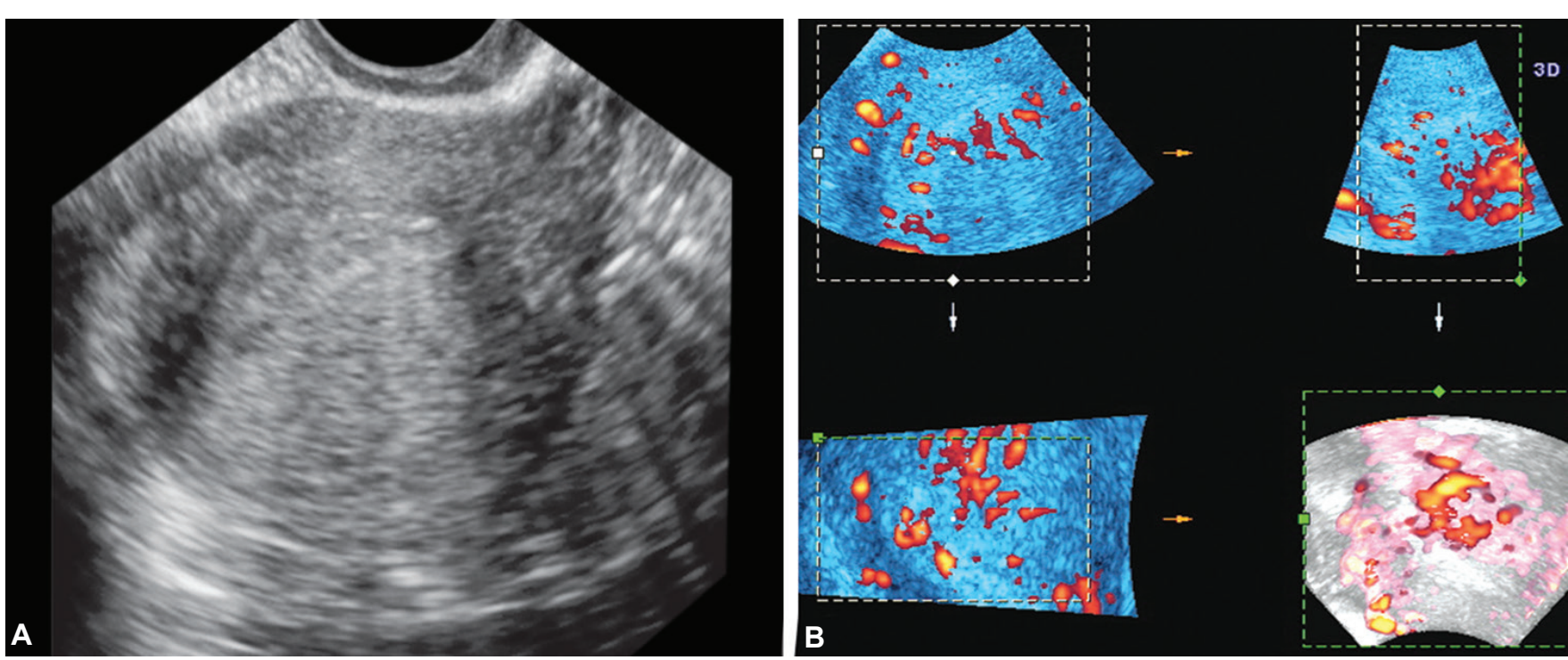

Figs 18A and B: Until the end of week 4, it is usually possible to observe transformation of the endometrium into decidua and its vascularization

\section{Fetal period}

Anatomy: From day 50 to 84 (8 to 12 full weeks)

\section{Ultrasonography: Until Day 98 (10 to 14 Full Weeks)}

Until week 10, the transvaginal route is universally recognised as the acoustic window for embryo study. After week 10, the transabdominal route may also be used with good ultrasound instruments in women with normal fat distribution. By week 10, CRL is no longer precise for dating pregnancy, acquiring an error of \pm 5 days (due to foetal flexion and extension). From week 11, biparietal diameter (BPD) is therefore preferred, though CRL is still important, for example in measuring nuchal translucence (NT).

By week 10, the foetus acquires definite anatomical characteristics that will persist for the rest of gestation, albeit with changes in size and function. By week 12, umbilical hernia disappears and by week 14 the YS is hardly visible and the two membranes fuse. In the brain, the choroid plexuses that almost filled the cranial cavity, are relegated to the posterior horns; the brainstem is increasingly evident and the posterior cranial fossa is readily detected by week $14 .{ }^{17}$ Although the corpus callosum ceases its formation later and is detectable by ultrasound by weeks 20 to 22, the cavity of the septum pellucidum can be detected. The heart with its four chambers becomes visible from week 12 transvaginally, however not in all cases. ${ }^{18}$ The detection percentage increases dramatically by week 14 and indeed more and more centres are delaying transvaginal screening for cardiopathy until weeks 14 to 16 . The fetal face is increasingly human and offers striking images by 3-4D. By week 12, BPD is the most reliable biometric value for dating pregnancy, having a mean error of \pm 1 week. (Figs. 22 to 24)

\section{Embryo-fetal Pathology Detectable by Ultrasound in the First Trimester}

The acoustic window of the first trimester of pregnancy (until week 14) offers the possibility of suspecting or diagnosing embryo-foetal malformations, chromosome anomalies and perinatal outcome. The possibilities in the first trimester have been described by Nikolaideset all who concentrated their research on the foetus from week 11 to 14 , documenting the many chromosome pathologies and malformations that can be detected. The fact that ultrasonographic diagnosis is becoming possible increasingly early in pregnancy partly attenuates the psychological problem of the mother and her partner when the painful question of whether or not to interrupt pregnancy arises. ${ }^{19}$ There have been many examples illustrating the significance of the first trimester for detecting chromosome and / or structural anomalies or at least for selecting populations at risk, whereas the second trimester is more indicated for malformations, for example the heart (for which there is ample documentation that suspected or actual diagnosis is possible in week, 14), spina bifida ${ }^{20}$ and Dandy-Walker syndrome. ${ }^{21}$ In any case, knowledge of the natural history of malformations is fundamental for understanding missed diagnoses. For example, partial or total agenesis of the corpus callosum cannot be diagnosed until week 22 to 24 , when the corpus callosum completes its formation. First trimester ultrasonography (weeks 11 to 14) is increasingly viewed as a time for morphological and structural check-up, similar if not better than second trimester scans, hence increasing use of the term first trimester sonoembryology, meaning the whole of the first trimester (Figs 25 to 30). 

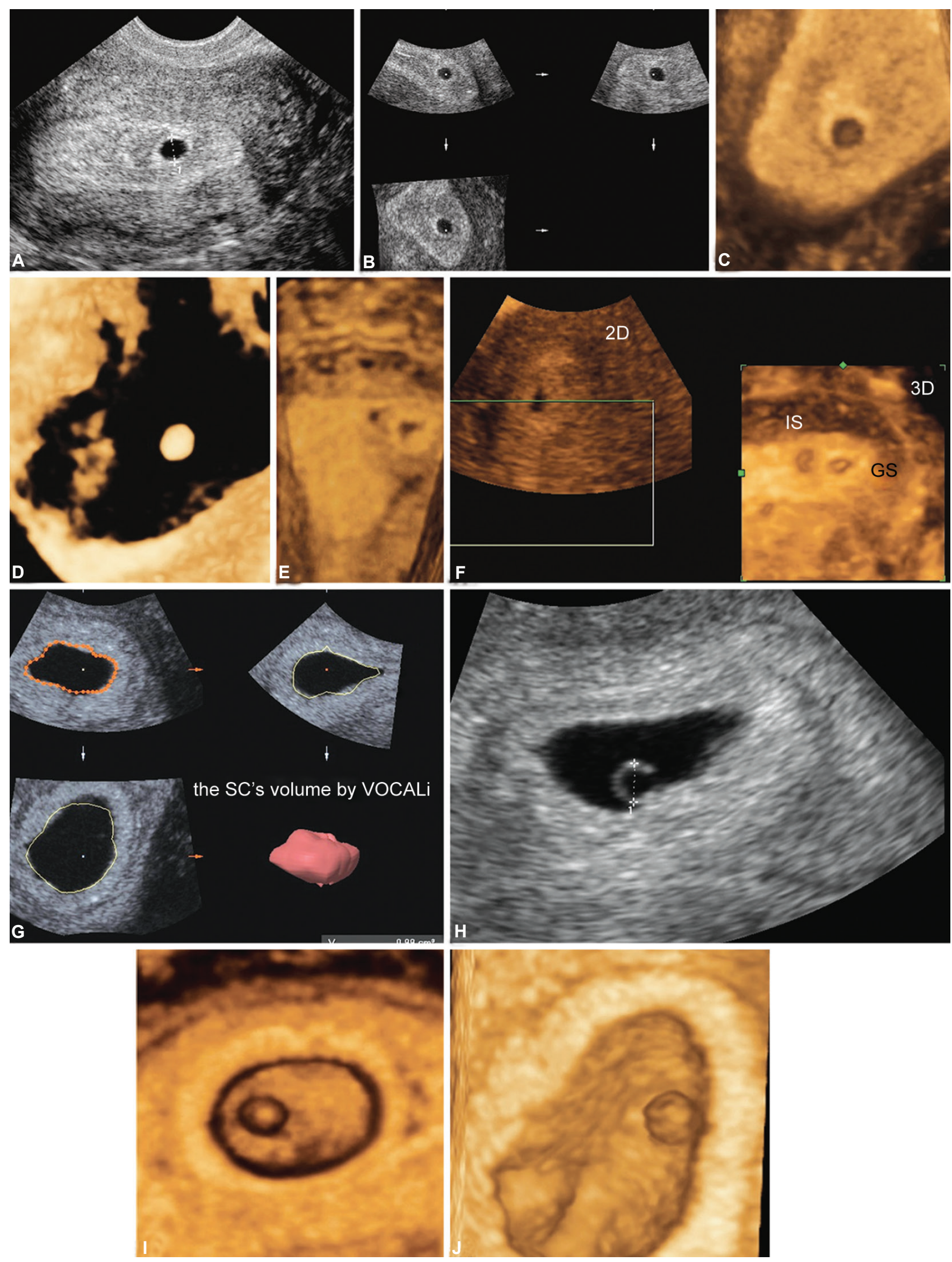

Figs 19A to J: Week 5: From gestational sac (GS) to yolk sac. The gestational sac is more evident by 3D, and it is possible to see the external wall of the GS, making it easier to distinguish the GS from the interdecidual space (IS) 

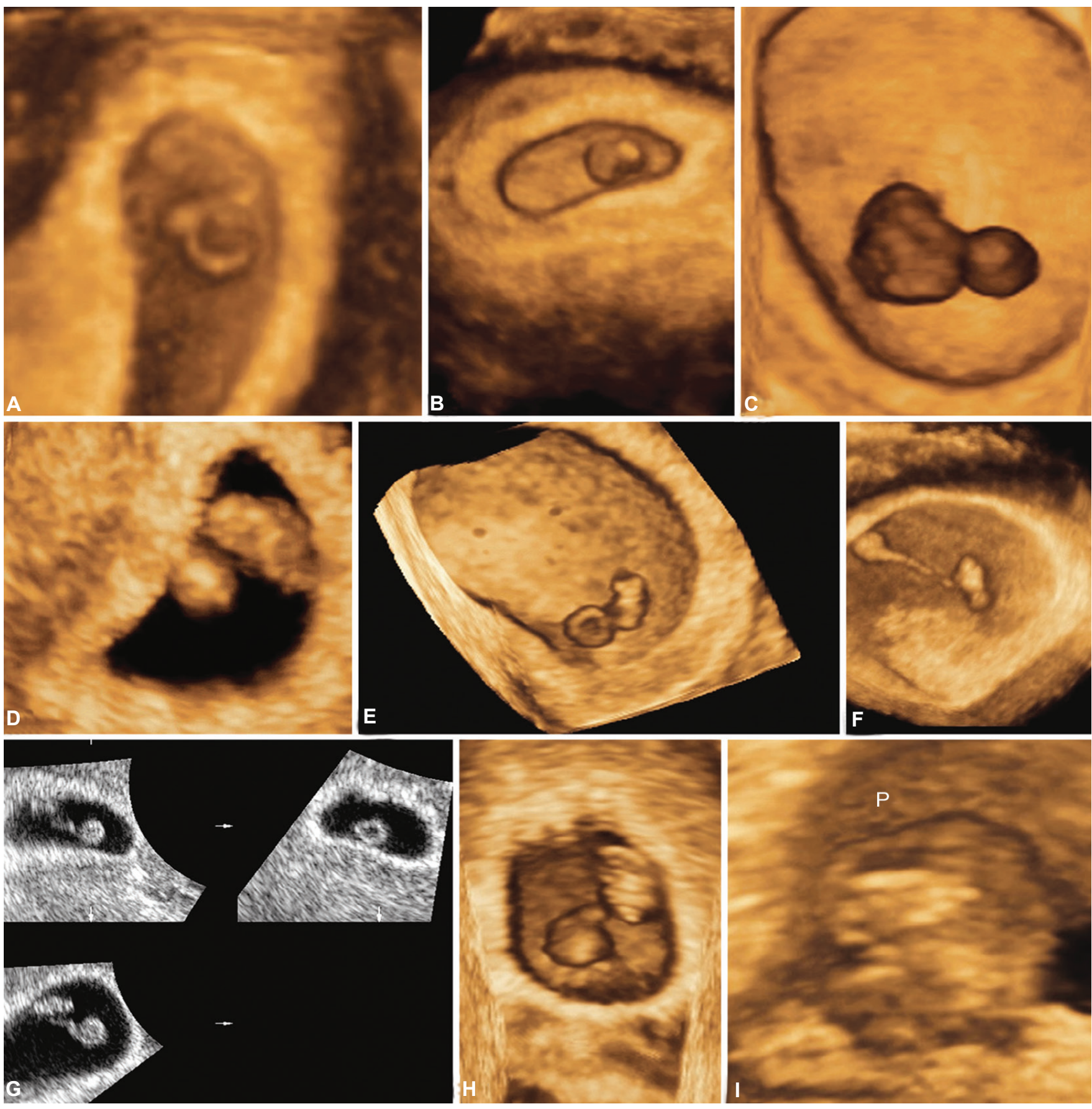

Figs 20A to I: (A to $\mathrm{H}$ ) Week 6: The embryo appears and the double bubble becomes more evident during the week. The embryo moves away from the yolk sac, which remains near the uterine wall and disappears in weeks 12-14; (I) Zoom of embryo head showing prosencephalon $(\mathrm{P})$
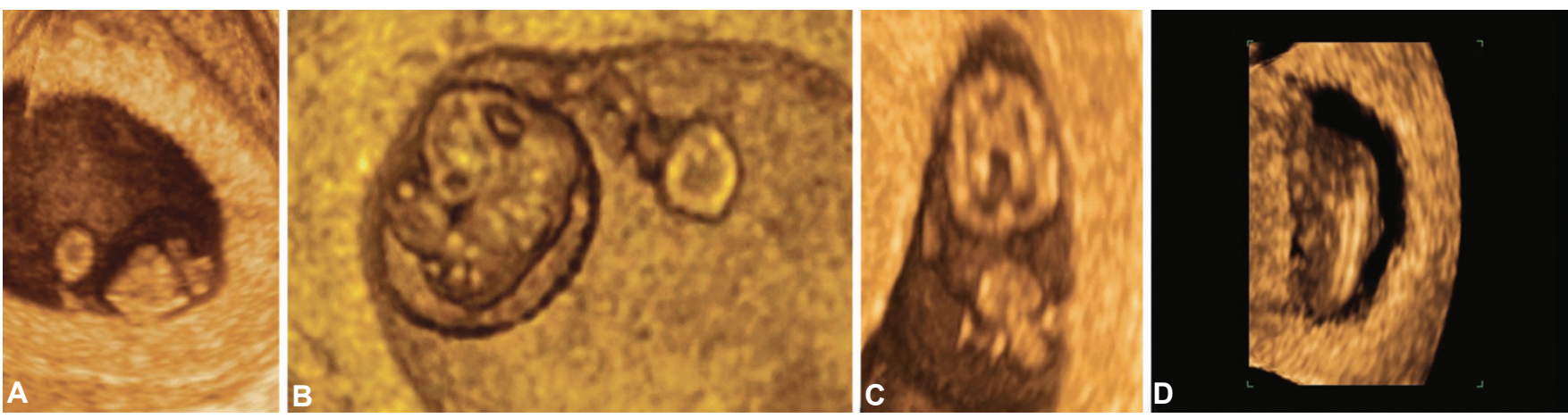

Figs 21A to D: Week 7 (6-12 mm): Embryo anatomy is more complex: note promesorhomboencephalon and first image of face. Arms and legs are present, as are the queue and the vertebral column. The amniotic membrane is well formed and divides the amniotic cavity from the celomatic cavity; the yolk sac lies between the amniotic and chorionic membranes 


\section{SIGNS PREDICTIVE OF ANEUPLOIDY AND STRUCTURAL EMBRYO-FETAL ALTERATIONS IN THE FIRST TRIMESTER}

It is common practice to obtain verbal or written informed consent before determining nuchal translucency. It also seems reasonable to make a similar contract (implying correct counseling and specific request) for pre dictive markers of aneuploidy in the first trimester, as is customary in the second trimester (so-called "genetic" ultrasonography). In my opinion, consent to scan for structural alterations, the only therapy for which would be interruption of pregnancy, is also advisable. Today, ultrasonography in pregnancy is a powerful instrument for serenity, but may also create gratuitous anguish. Before carrying it out, it is important to discuss it with the woman, not only specifying the limits of the method and of the operator, but also asking clearly what the women expects and wants from the scan.

\section{TRANSITORY SIGNS}

\section{Nuchal Translucency}

The problem with screening tests in which nuchal translucency (NT) is a constant component is too complex to discuss in an atlas, however the detection of this transient sign is easier and quicker with multiplanar than with classical 2D. (Figs 31 and 32) The current scientific findings have shown that can detect even an intracranial translucency (fourth ventricle) that seems to offer a sensitivity and specificity of nearly $100 \%$ in the early detection of spina bifida ${ }^{22,23}$ (Fig. 16).

At 11 to 13 weeks' gestation, during the first trimester screening of chromosomal abnormalities in the mid-sagittal view of the fetal face we can to obtain the nuchal translucency thickness and the nasal bone view; in this view is visible the fourth ventricle. In the normal fetuses the fourth ventricle was always visible and the median anteroposterior diameter increased from $1.5 \mathrm{~mm}$ at a crown-rump length (CRL) of $45 \mathrm{~mm}$ to $2.5 \mathrm{~mm}$ at a CRL of $84 \mathrm{~mm}$. In the fetuses with spina bifida the fourth ventricle space was compressed and no could be seen, we can also be used for early detection of open spina bifida. ${ }^{24}$

Pending clinical confirmations is important to be able, during a 11 to 14 week scan, to detect these markers that can be considered as an alarm bell to identifying cases at risk assessment of intracranial translucency (IT) in the detection of spina bifida at the 11 to 13-week scan. (Fig. 23 bis)

\section{Ductus Venosus}

Measurement of NT in the first trimester has become a consolidated method for identifying fetuses at risk for
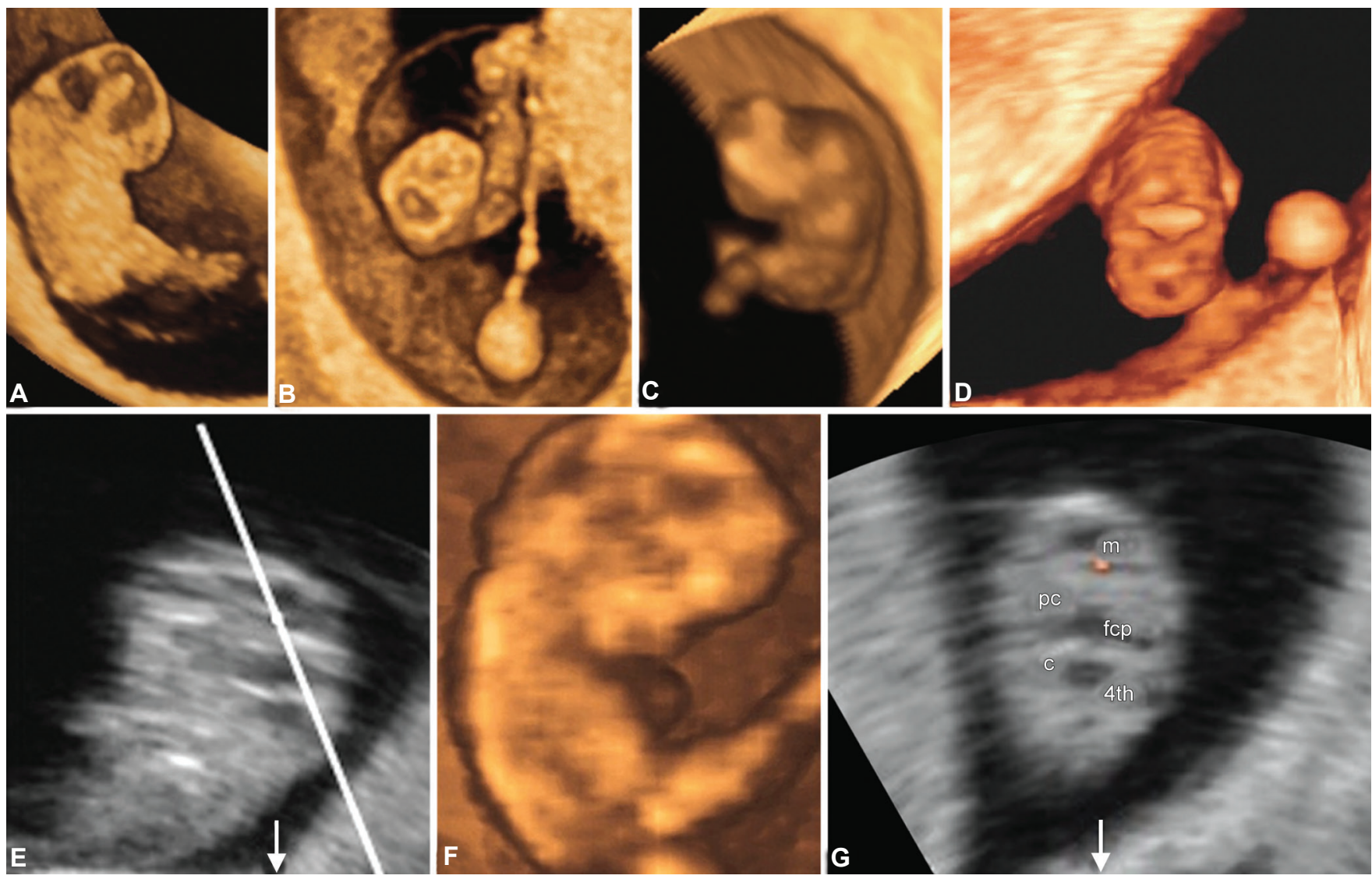

Figs 22A to G: Week 8 (22 mm): Head structures are better defined (Abbreviations: 4th, fourth ventricle; c, cerebellum; pcf, posterior cranial fossa; $\mathrm{cp}$, choroid plexus; m, mesencephalon) 

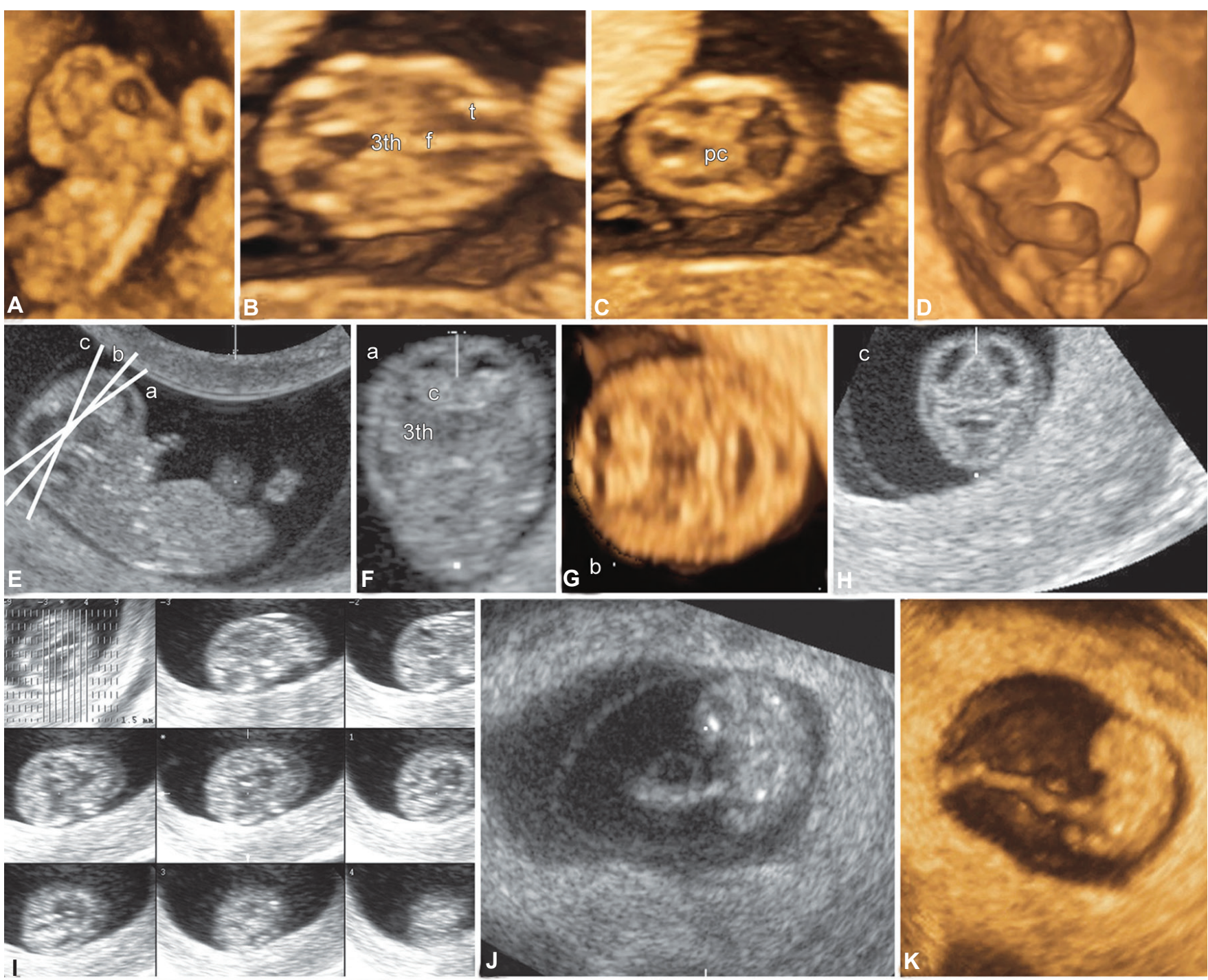

I
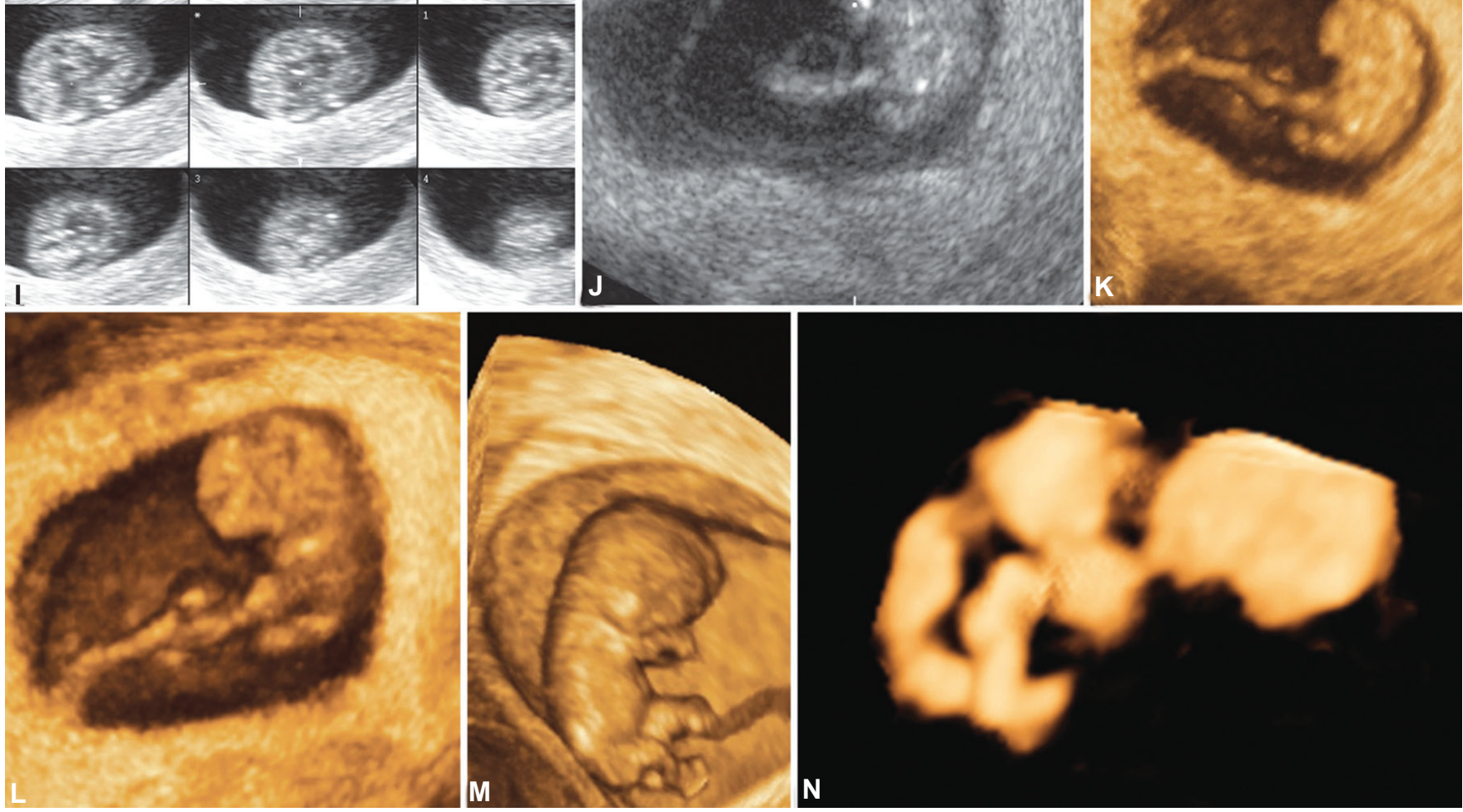

Figs 23A to N: Week 9 (29 mm): (A to M) Embryo acquires human features and external morphology is now fixed. Detection of physiological umbilical hernia. Complete view of brain clearer with tomography ultrasound imaging which makes ultrasonography similar to NMR (the image can be cut in real time up to 18 times at predetermined distances); (N) Invert mode shows spatial extent of ventricles

chromosomal abnormality. The high percentage of heart defects in fetuses with increased NT, whether isolated or caused by chromosome anomalies, has stimulated considerable interest. The association, together with echo-Doppler modifications of the ductus venosus (DV) in fetuses with increased NT, suggests that altered heart function could play a role in determining an increase in $\mathrm{NT}^{25,26}$

In particular, inverted flow in the DV during heart's contraction (A wave) has been associated with increased NT. The DV is a communicating vessel that carries well oxygenated blood of the umbilical vein into the 

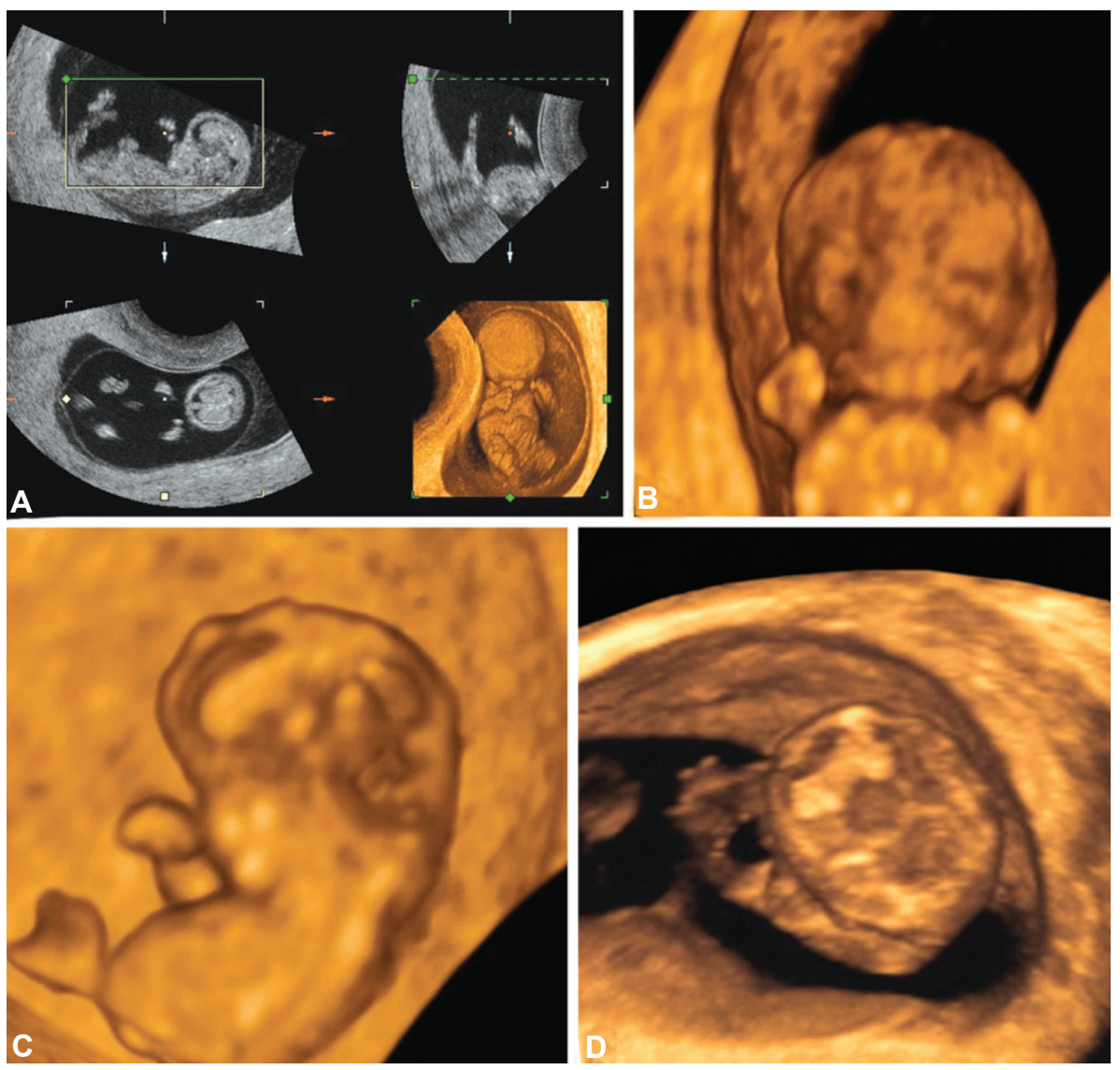

Figs 24A to D: Week 10: Beginning of fetal period. Body organs have formed and will grow, and acquire organ functions. Multiplanar view of $34 \mathrm{~mm}$ fetus, showing face, ventricles and cerebellum
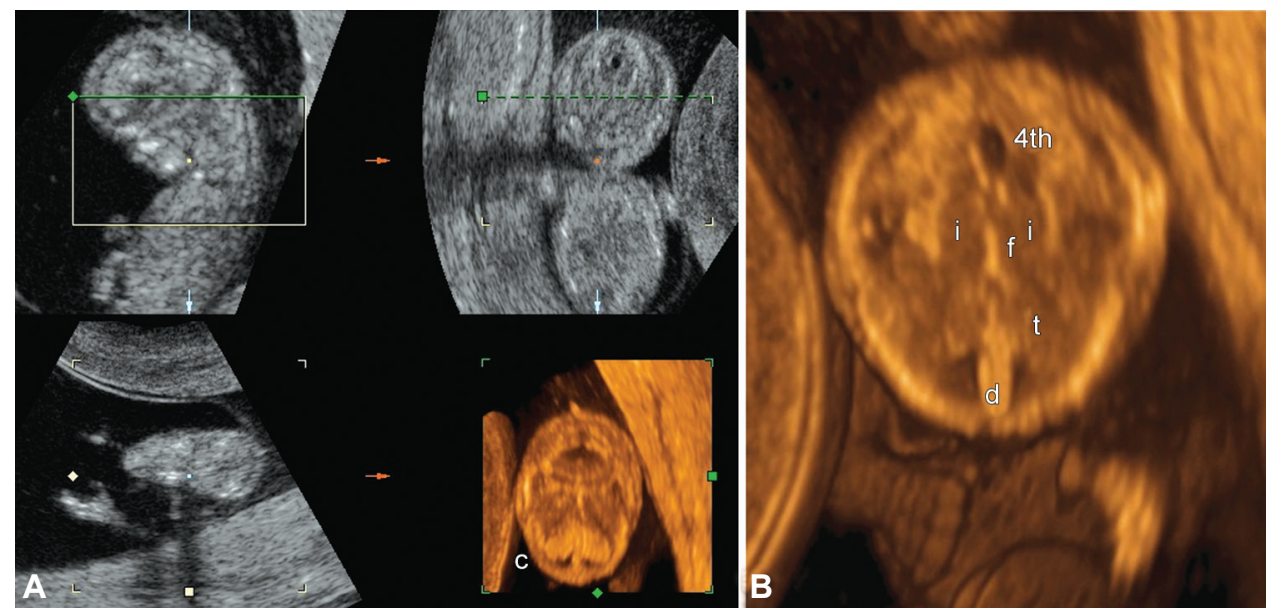

Figs 25A and B: Weeks 11-12: Multiplanar view showing brain

(Abbreviations: 4th, fourth ventricle; $\mathrm{f}$, falx; $\mathrm{H}$, hypothalamus; $\mathrm{d}$, diencephalon; $\mathrm{t}$, telencephalon)

right atrium, through the oval foramen. This vessel is important for assessing the presence of heart function anomalies. Blood flow in the DV is characterised by high velocity during ventricular systole ( $\mathrm{S}$ wave) and diastole (D wave) and also by forward flow during atrial contraction (A wave). In heart dysfunction due to heart defects, the A wave is absent or negative.

Assessment of flow in the DV could play a role in secondary screening, permitting further reduction in the percentage of false positives in screening for chromosome anomalies in the first trimester. ${ }^{27,28}$ A major association has been demonstrated between chromosome anomalies and abnormal blood flow in the DV at 11 to 14 weeks of pregnancy in high risk pregnancies. ${ }^{29,30}$ DV flow anomaly associated with heart defects and adverse outcome of pregnancy has also been observed. Some authors suggest that the DV can be an important prognostic factor in fetuses with increased NT and normal kariotype.

In foetuses with chromosome anomalies, whose parents decided to continue the pregnancy, heart dysfunction has been found to be a temporary condition: transitory anomalous flow in the DV, detected by colour 

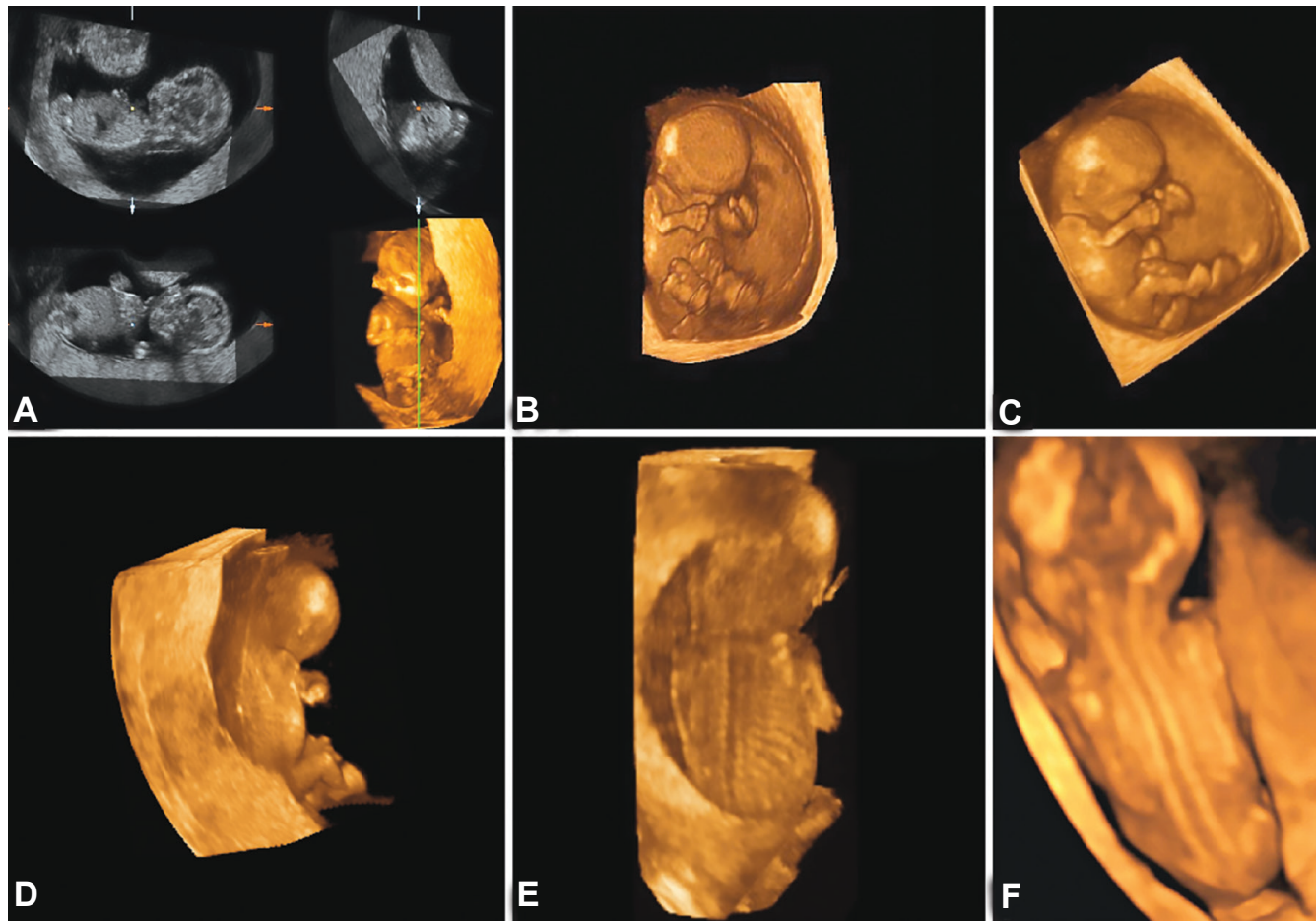

Figs 26A to F: Weeks 13-14: Fetal morphology is clear; note details of face, limbs, fingers, toes and neck. Detailed study of spine is possible

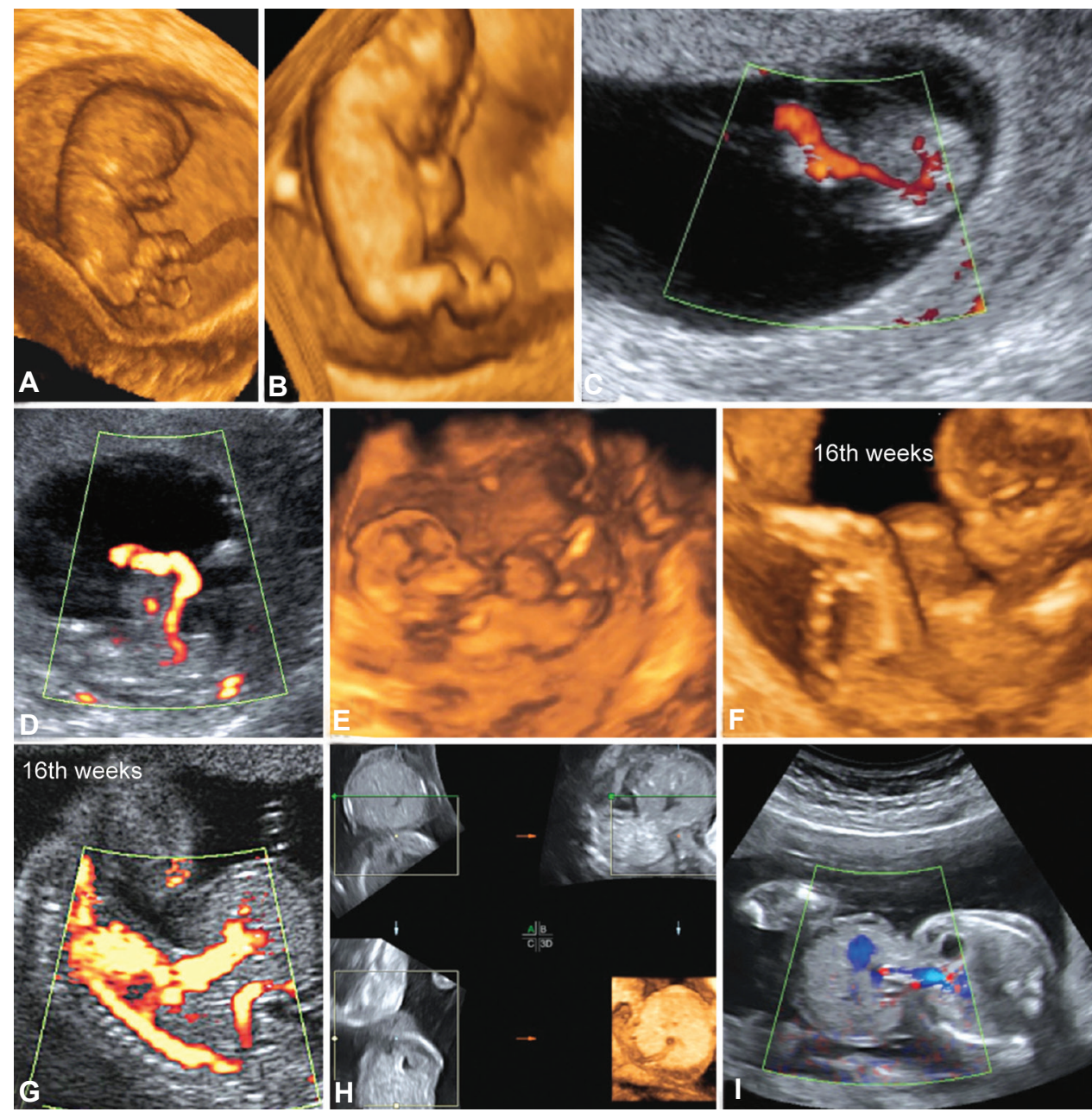

Figs 27 A to I: Omphalocele at weeks 13,16 and 18. Note difference between normal and abnormal hernia in A and B: umbilical artery starts from top of hernia (color and power glass body mode). Week 16 and 18 images show homogeneous echogenicity of small intestine and stomach 

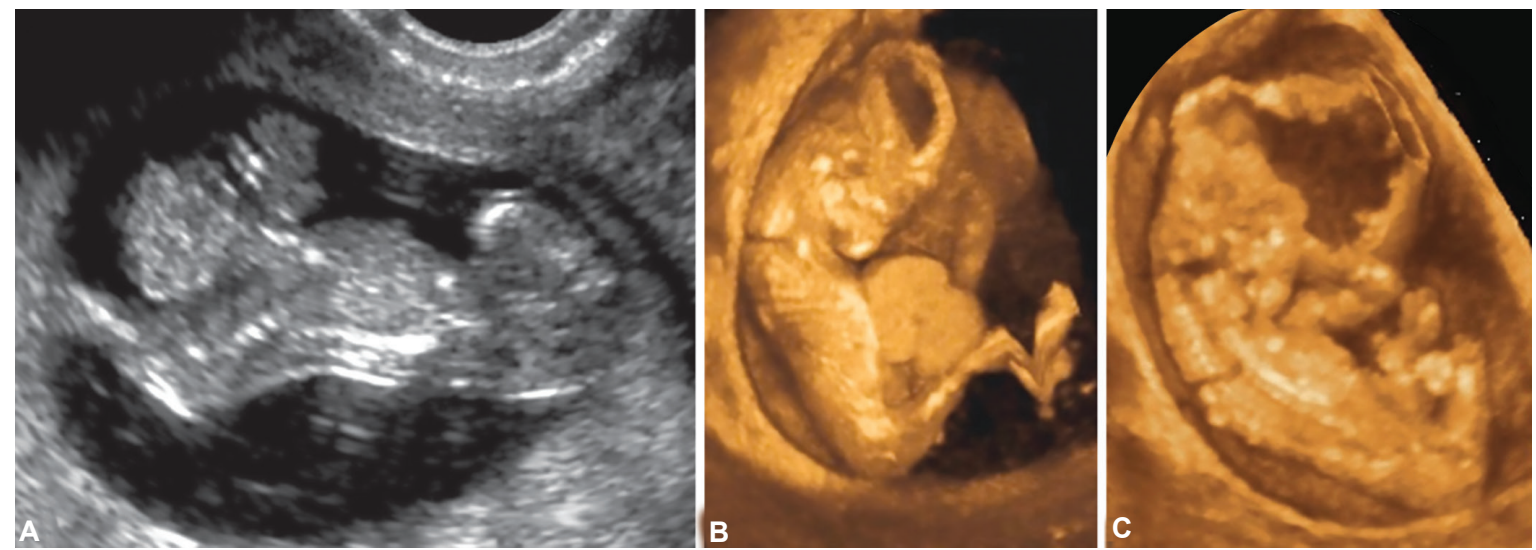

Figs 28A to C: Alobar holoprosencephaly associated with omphalocele in a $46, \mathrm{XX}$ fetus at week 12 . The 3D provides more information than $2 \mathrm{D}$
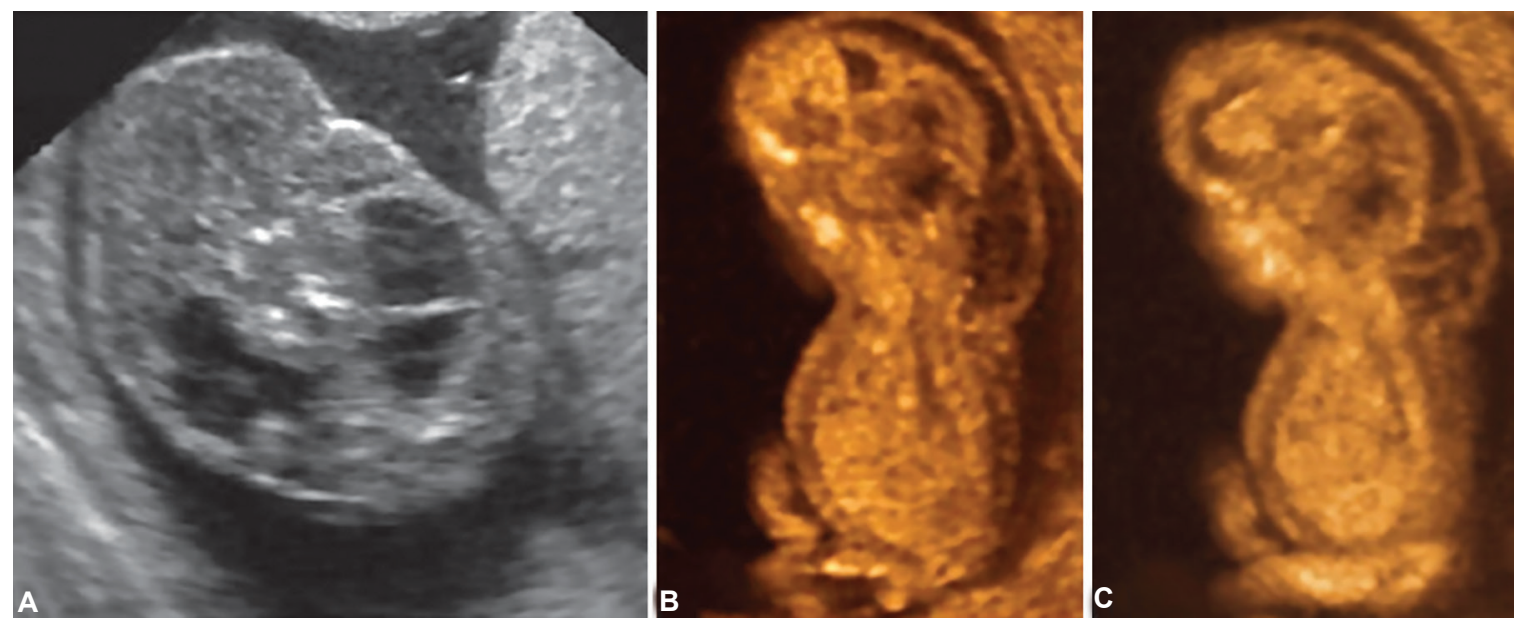

Figs 29A to C: Septate cystic hygroma at week 13 as shown by 3D and 2D
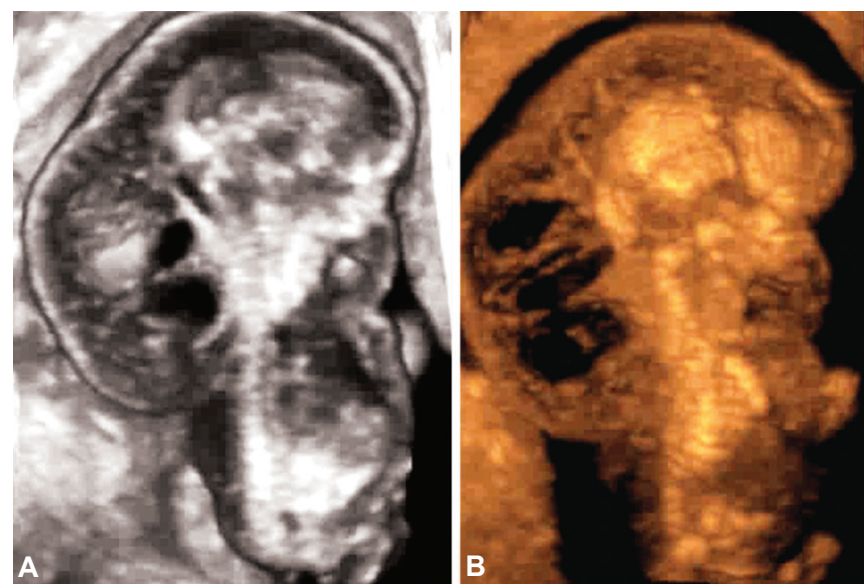

Figs $30 \mathrm{~A}$ and B: Hygroma and fetal hydrops at 16 weeks. The hygroma plurisepimentato extends back to the fetus

Doppler, manifested as "reversed flow" during contraction. This marker is detected early, around week 13, and usually disappears by week 20. The dysfunction that caused it aggravates retronuchal oedema and may culminate in foetal hydrops, which could explain some cases of intrauterine foetal death. At 10 to 13 weeks +6 days the duct anomaly is therefore associated with aneuploidy ( $80 \%$ of Downfetuses versus $5 \%$ of euploidfetuses), heart malformations and unfavourable outcome of pregnancy. To study the DV, 3-4D does not offer particular advantages, whereas 2D is fundamental and exclusive for correct detection (Fig. 34).

\section{Nuchal Edema, Cystic Hygroma and Non- immune hydrops}

Subcutaneous edema detected sonographically in the forms of nuchal edema, cystic hygroma, or non-immune hydrops may be a sign of chromosomal abnormalities. ${ }^{31}$

Cystic hygroma is a congenital malformation of the lymphatic system characterised by a thin walled cystic 

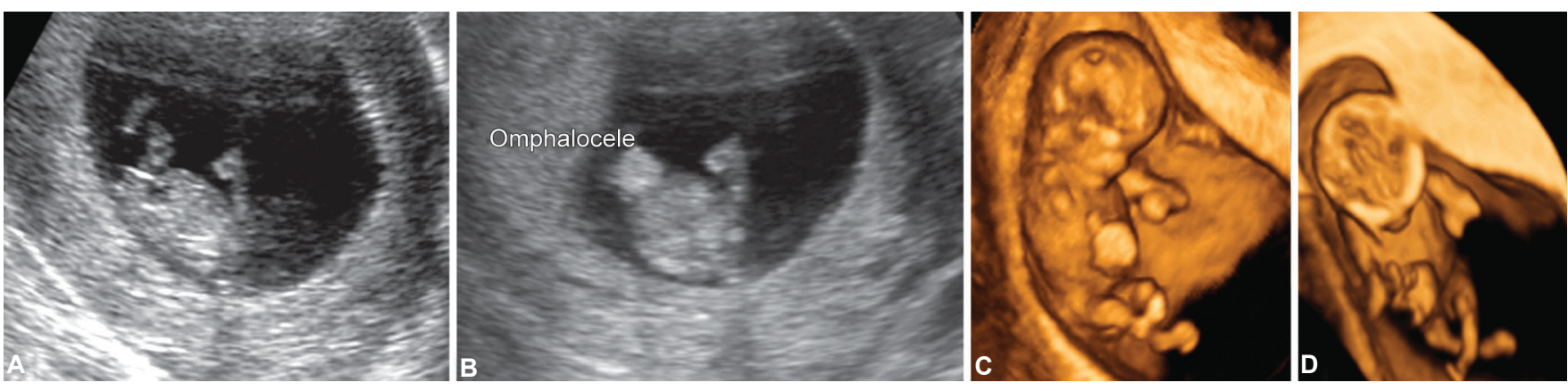

Figs 31A to D: Radius-ulnar agenesis with omphalocele in fetus with trisomy 18 at week 10

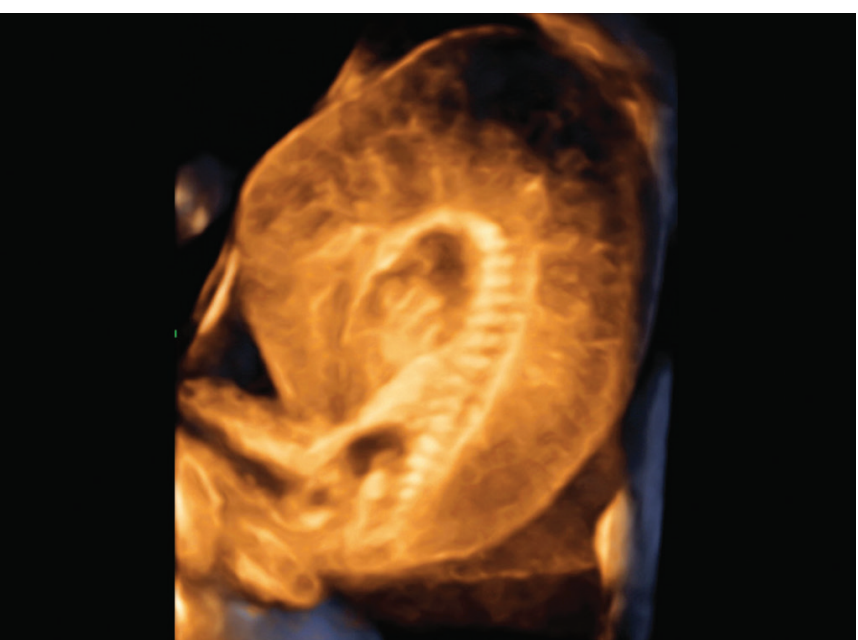

Fig. 32: Twin reversed arterial perfusion syndrome (TRAP). (A) Acardius acephalus (13 weeks) is characterized by amorphous shape of the cephalic pole, the upper limbs are absent; the lower limbs are present and the intrathoracic and abdominal organs are rudimentary, with diffuse subcutaneous edema

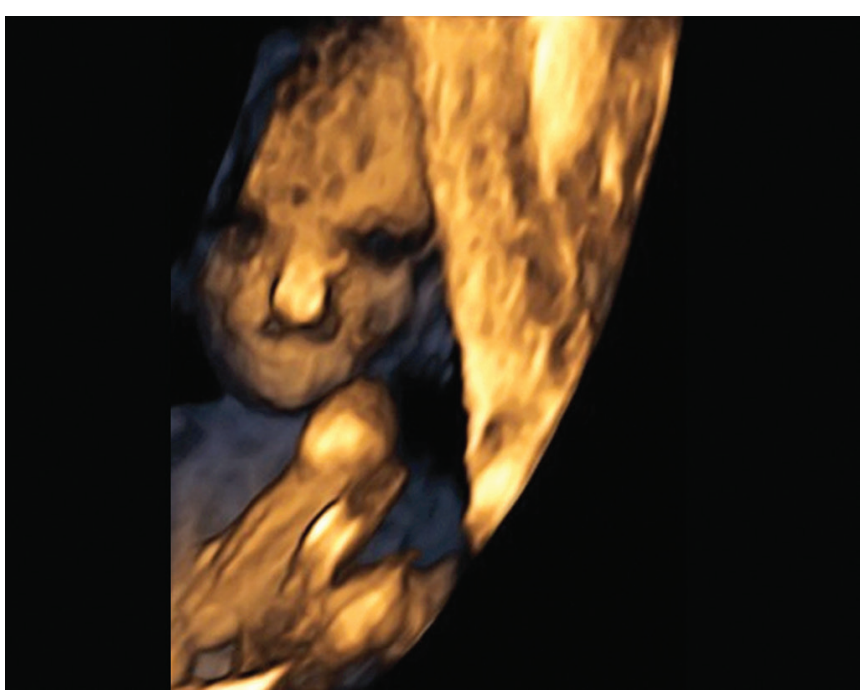

Fig. 34: Frontal holoprosencephaly. Elephant fetus at 11 weeks

structure full of liquid. It is usually situated in the nuchal region, extending from the superior occipital bone, caudally and medially to the sternocleidomastoid muscle. It consists of two symmetrical cavities completely separated by a median nuchal ligament and is distinguished from other craniocervical masses, such as encephalocele, meningocele, teratoma, hemangioma and retronuchal oedema. ${ }^{32,33}$

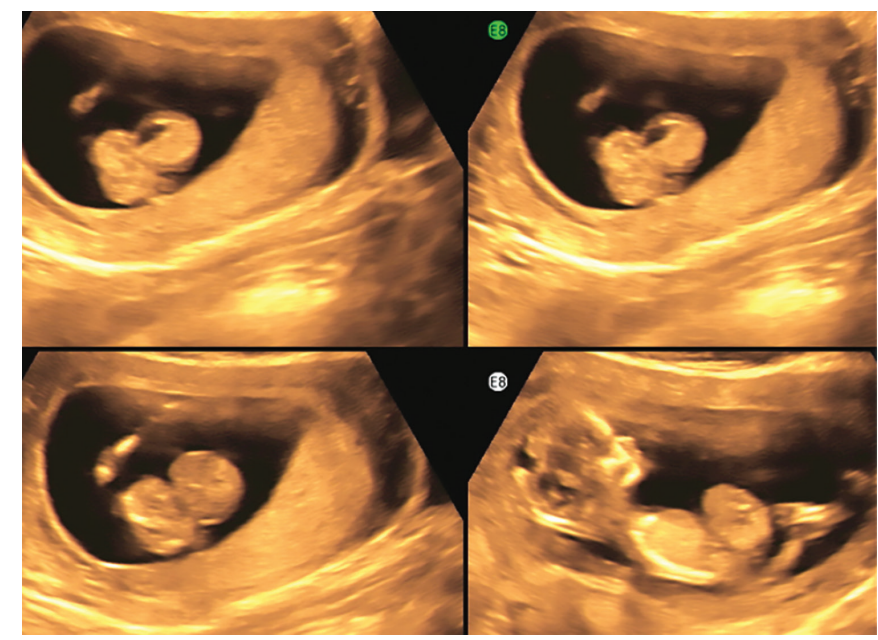

Fig. 33: Omphalocele at 12 weeks

At about 40 days of embryo development, the jugular lymphatic sac forms a connection between the jugular duct and the internal jugular veins which become the terminal portions of the lymphatic and thoracic ducts. According to the theory of obstruction of the jugular lymphatic sac, the connection between the jugular lymphatic sac and the jugular veins does not form in the case of cystic hygroma and the lymph builds up in tissues around the neck, causing the sac to swell. Cystic hygroma is often detected by prenatal ultrasound, though some cases develop in the postnatal period, with an incidence of about $1 \%$. The defect may regress spontaneously during pregnancy, leaving extra skin in the neck region, or progress dramatically towards a form of generalised foetal hydrops. ${ }^{34}$

Fetal cystic hygroma was usually readily diagnosed by transabdominal ultrasound in the second trimester of pregnancy. Today transvaginal ultraound has increased the percentage of diagnoses in the first trimester. ${ }^{35}$ Ultrasound diagnosis of cystic hygroma is based on visualisation of a prominent anechogenic or hypoechogenic area (more than $3 \mathrm{~mm}$ thick) in the occipital, nuchal or upper thoracic regions, bilaterally. On the contrary, physiological build-up of fluid or the nuchal bleb is generally considered normal when it is less than $3 \mathrm{~mm}$ thick: these build-ups seem to occur in $40 \%$ of embryos before week 10 and disappear at week 11. 
Cystic hygroma may be single or consist of multiple cysts and is classified as septate and non septate. Though confused for years by various authors, this distinction is important for prognosis, because septate cystic hygroma is strongly associated with aneuploidies. ${ }^{36}$

The presence of cystic hygroma in the first trimester is associated with increased risk of fetal chromosome anomalies: about $50 \%$ of fetuses with ultrasound diagnosis of cystic hygroma in the first trimester also have a chromosome anomaly. A high percentage of the cases undergoing cytogenetic analysis show Turner syndrome, often associated with trisomy 21,18, 13 or other structural anomalies. The risk of this transient anomaly increases with increasing maternal age.

As yet there are no ultrasonographic elements for distinguishing cystic lesions that may regress from those that persist. However, when the nuchal cyst is small and non septate and karyotype is normal, it usually resolves spontaneously with good foetal outcome. When septate cystic hygroma is diagnosed early in pregnancy, the risk of chromosome aberrations is much greater and the outcome is therefore worse, also because septate hygroma is often larger and frequently develops into foetal hydrops. Spontaneous regression of cystic hygroma has been reported in chromosomally normal fetuses and in, down, Turner and Robert syndrome fetuses.

Fetuses diagnosed with non septate and septate forms, with or without associated malformations, are at high risk of chromosome aneuploidies. The mothers need correct counseling to assess the possibility of cytogenetic tests to determine fetal karyotype, to programme subsequent ultrasound monitoring to exclude morphological anomalies, particularly of the heart and circulatory system, and to evaluate any worsening of edema or its resolution. Fetuses with normal karyotype are usually free of hygroma by weeks 16 to 17 and many have normal phenotypes at birth. Ultrasound parameters such as hygroma size and the time of its disappearance associated with fetal echocardiography may be useful to identify pregnancies at risk for dysmorphic conditions such as Noonan syndrome (Fig. 27).

\section{PERSISTENT SIGNS}

\section{Nasal Bone}

In 1866, Langdon Down noticed that subjects with trisomy 21 had a small nose. An anthropometric study on 105 Down patients confirmed that the nasal bone (NB) was smaller than normal in $49.5 \%$ of cases. Recent radiological and ultrasound studies have shown that nasal hypoplasia is already present in the uterus. ${ }^{37,38}$ A study of 105 fetuses with trisomy 21, aborted at weeks 12-25, showed lack of ossification of the NB in $32.4 \%$ of cases and hypoplasia in
$21.4 \%$. The correlation between absence of NB at weeks 11 to 14 and increased risk of trisomy 21 was reported for the first time in 2001. Indeed, about $65 \%$ of Downfetuses lack, or have a small, NB.

The nasal bone can be seen by scan from week $11 .{ }^{39}$ Many recent studies show a strong association between absence of NB at this gestational age and trisomy 21 and other chromosome anomalies. ${ }^{40,41}$ We can therefore say that at 11 to 13 weeks +6 days, fetal profile can be examined correctly in more than $95 \%$ of cases and that the NB is absent in about 70\% of fetuses with trisomy 21 and in about $55 \%$ of fetuses with trisomy 13 . The incidence of absence of NB in euploidfetuses is less than $1 \%$. Absence of NB is therefore a major marker of trisomy 21. There are some limiting factors in the ultrasonographic assessment of NB. ${ }^{42,43}$ Between weeks 11 and 14 from the last menstrual cycle, the probability of visualising the NB increases with increasing gestational age. The presence of NB is not independent of fetal nuchal thickness, since the possibility of visualising it seems to decrease with increasing NT. Finally, ultrasound detection of NB at 11-14 weeks requires much technical skill of the operator, unlike later in pregnancy. ${ }^{44}$

Absence of NB has a higher incidence in fetuses of African origin than in Caucasians and decreases with CRL. In calculating individual patient-specific risk of Down syndrome, it is necessary to consider these demographic and ultrasonographic aspects.

If examination of foetal profile to detect NB is associated with NT and maternal serum proteins (free beta hCG and PAPP-A) during first trimester screening for trisomy 21 , the detection rate can increase substantially and the false positive rate decrease. For a false positive rate of about $5 \%$, the detection rate increases from about $75 \%$ (NT) and $90 \%$ (NB + maternal serum proteins) to $93 \%$ $(\mathrm{NT}+\mathrm{NB})$ and $97 \%(\mathrm{NT}+\mathrm{NB}+$ maternal serum proteins). For a false positive rate of $1 \%$, the detection rate could be about $57 \%$ for NT, $86 \%$ for NT + NB and $93 \%$ for NT, $\mathrm{NB}$ and maternal serum proteins.

In conclusion, the absence of NB or small NB are more common in fetuses with chromosome anomalies. It is therefore clear that NB is becoming a major marker in the prenatal diagnosis of aneuploidies.

There is a statistically significant difference between detection of NB by 2D and 3D techniques. Traditional scan is about $20 \%$ less reliable in detecting presence/ absence of NB and also for the number of bones detected. This difference can be as high as $40 \%$ when $2 \mathrm{D}$ is performed with mediosagittal scan of the fetal face, since detection of the NB requires a longitudinal foetal scan as for detection of NT, but in the case of NB the section is 1 to $2 \mathrm{~mm}$ from the sagittal median. Further studies seem to suggest greater reliability in the second trimester, with 


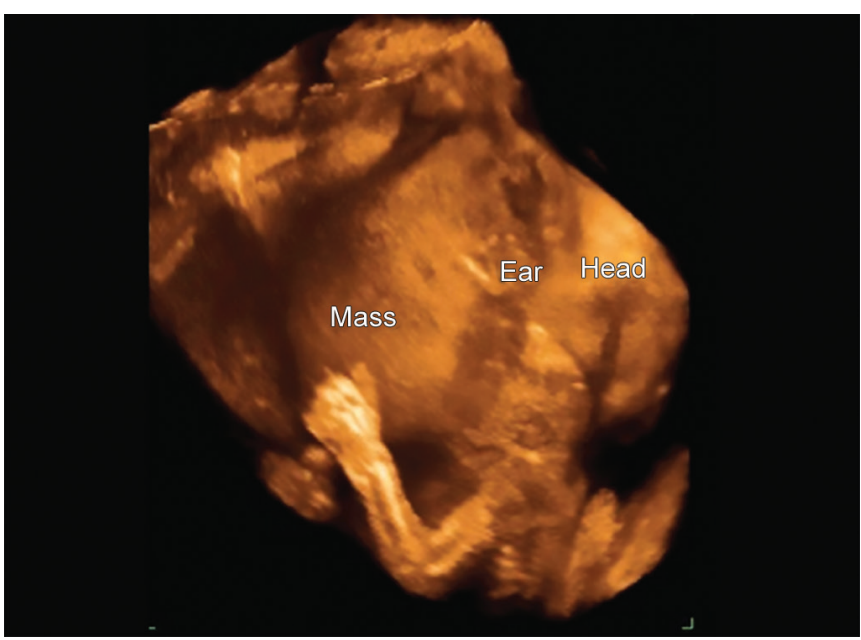

Fig. 35: Epicanto at 14 weeks. The mass distance morphology of the face

a cut-off of $2.5 \mathrm{~mm}$ for NB and the presence of both nasal bones; in the second trimester, 3D plays an important role, making detection of the bones simpler and more reliable $^{45}$ (Fig. 35).

\section{Palate}

It is possible investigate the normality of the secondary palate better than the primary palate because it is very difficult define a normality of the lips in the first trimester (foto 14 settimane). We can detect the secondary palate by two methods: by the evidence of the retronasal angle with a $100 \%$ confidence by the authors) using 2D or 3D multiplanar or omni-view and by the Faure method with a coronal/sagittal 3D scan of the foetus's profile and rotation of the picture avoiding the maxillary shadow and maximum or minimum rendering of the palate by axial section (delta sign) (Fig. 35).

\section{Jaw Bone}

In a series 89 Down fetuses compared with a population of 900 euploidfetuses, Cicero (2004) found jaw bones shorter by about $0.7 \mathrm{~mm}$ (with variations in growth from 4.8 to $8.3 \mathrm{~mm}$ from 11 to 14 weeks) in fetuses with NB and 0.5 $\mathrm{mm}$ in fetuses without NB. ${ }^{46}$ This characteristic is specific to trisomy 21 and not detectable in other aneuploidies. It is important to assess the jaw correctly by sagittal scan taking the mandibular condyle as reference point.

\section{Single Umbilical Artery}

A single umbilical artery in the first trimester of pregnancy is associated with trisomy 21 and especially trisomy 18. Nikolaides et al. showed that an umbilical artery is missing in $77 \%$ of cases of trisomy $18^{47}$ (Fig. 33).

The screening tests for Down syndrome in the first trimester with the use of NT and other marker (nasal bone, ductus venosus) have focused the investigator's attention to the first trimester 11 to 14 week and today it's possible to diagnose about the $50 \%$ of structural foetal malformation that we can detect in the second trimester ${ }^{48}$ (Figs 26 to 29).

\section{SECOND AND THIRD TRIMESTERS}

The scan performed in the second trimester, usually around week 20 (range week 18 to 23 , depending on the protocol), is used to study foetal anatomy and detect foetal malformations, as well as to evaluate foetal growth by means of biometric parameters. ${ }^{49}$ Despite the high expectations of pregnant women about the diagnostic capacity of ultrasound (presumably due to incorrect information in the media and often also from specialists), the various studies on detection of fetal malformations by standard ultrasound in the second trimester show rates that do not exceed 40 to $60 \%$ of all malformations detected at birth, with a homogeneous mean prevalence of $2.5 \%$. The Eurocat report, ${ }^{50}$ for example, documents a diagnostic capacity of $62 \%$ for 11 major pathologies among 4366 malformations in 1,198,519 babies born in 17 European regions in the period 1995 to 1999. Variability was high, ranging from $25 \%$ in Croatia to $88 \%$ in Paris, with enormous regional differences. Moreover, about 30 to $40 \%$ of cases were diagnosed after week 24 , for various reasons, not least of which the natural history of malformations. No malformation was diagnosed in all carriers; for example, anencephaly was diagnosed in $94 \%$ of cases. It is therefore necessary to be precise and careful when informing women about the intrinsic limits of general ultrasound, as well as individual limits determined by the type of equipment and operator experience. As mentioned for ultrasound in the first trimester, the sophistication of instruments and operator experience also make very accurate and difficult diagnoses possible in the second trimester. Indeed, today the study of foetal anatomy can be much more detailed than in the 1990s. Thus greater experience, attention to districts such as the face, heart and circulatory system, should improve the sensitivity of diagnosis of malformations, though this quite reasonable claim has not yet been demonstrated. Specialists in prenatal diagnosis can also achieve great morphological detail with the aid of sophisticated new instruments with 3-4D technology. ${ }^{51}$ For specific use of these instruments, we consider the various systems and organs.

\section{Head}

The head offers the possibility of detailed examination of a series of morphological signs by 3-4D. ${ }^{52-54}$. The face is accessible and it is relatively easy to exclude or diagnose 


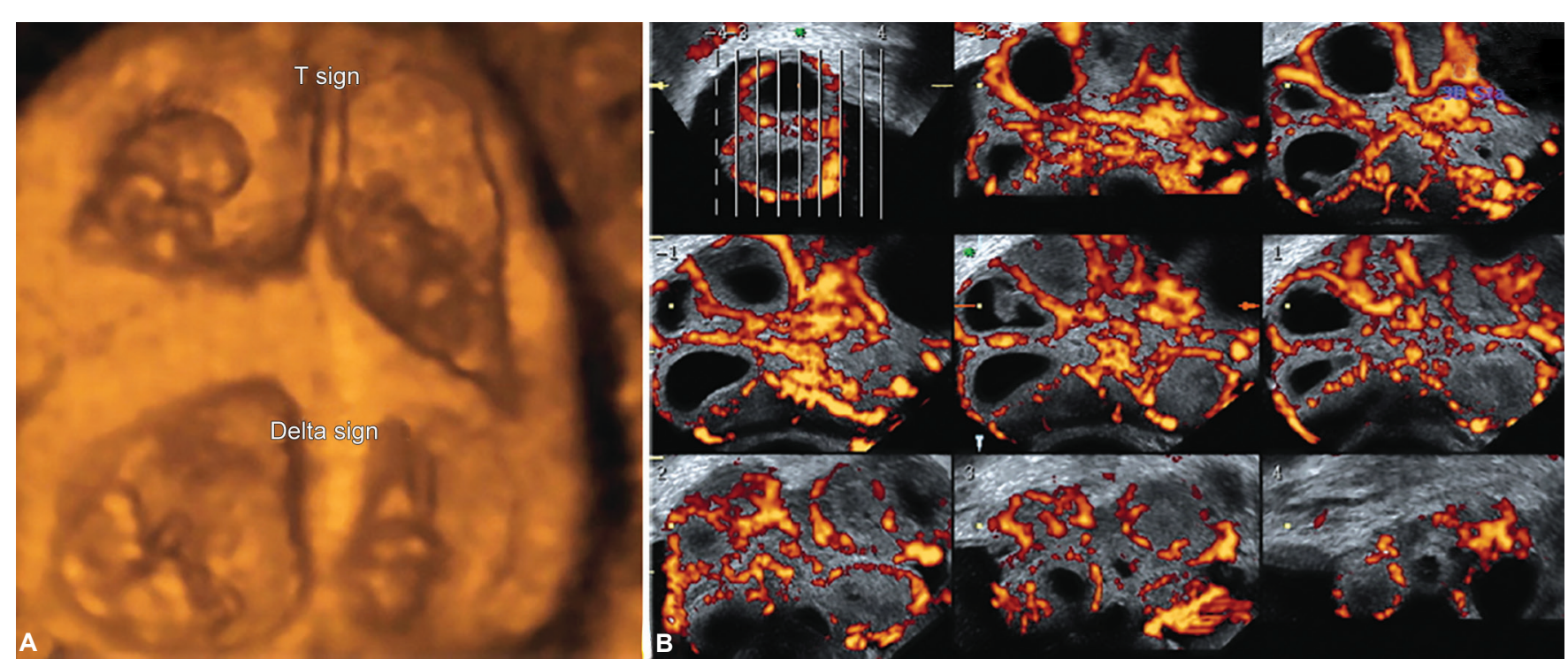

Figs 36A and B: Unusual scan of quadrigeminal pregnancy arising from FIVET of three oocytes: two twins are clearly bichorionic biamniotic (delta sign) and two monochorionic-monoamniotic (T sign) and a distribution of vessels in multiple pregnancy in the first trimester

cleft lip in this period. ${ }^{55}$ Note that $3 \mathrm{D}$ technology includes $2 \mathrm{D}$ as base, so the capacity to visualise the foetal face by $3-4 \mathrm{D}$ in the second trimester is certainly much better than with 2D, with due enhancement and limitation related to foetal position, maternal abdominal fat, the placenta and quantity of amniotic fluid. ${ }^{56}$ Given time (and perhaps more than one session) it is possible to obtain a good image of the foetal face in almost all cases in the period 19 to 23 weeks in 85 to $90 \%$ of cases (our personal percentage for 3540 pregnancies is $92 \%$ ). In the next period of pregnancy, the possibility of exploring the foetal face decreases as pregnancy proceeds. Indeed, in the third trimester, after week 35 , the face can only be visualised in $30-50 \%$ of cases and sometimes requires two or three sessions in the case of suspected diagnosis (Fig. 36).

Operator experience is critical. After initial successes, considerable difficulty is often encountered in analysing a saved volume: to obtain good images, 3D takes time and application as well as interest and predisposition. 3-4D is much less instinctive than 2D, which gratifies the operator with interpretable images after a few hours of practice (for example, measurement of BPD). The first impression of a face naturally cannot have scientific value, though many facial dysmorphisms depend on genetic syndromes and aneuploidies. Hyper and hypotelorism, a weak chin and low ear position are easily and immediately detectable by $3 \mathrm{D}$ and this prompts us to consider the foetus as if it were a newborn. Moreover, with 4D we can observe sucking movements and attitudes, yawning, extraflexion of the tongue and movements of the hands, arms and legs, all characteristic of fetal wellbeing. ${ }^{57}$ Maximum mode rendering enables exact views of bones, ${ }^{58}$ detailed study of cranial bones and sutures, as well as measurement and counting of nasal bones makingeasier a diagnosis of
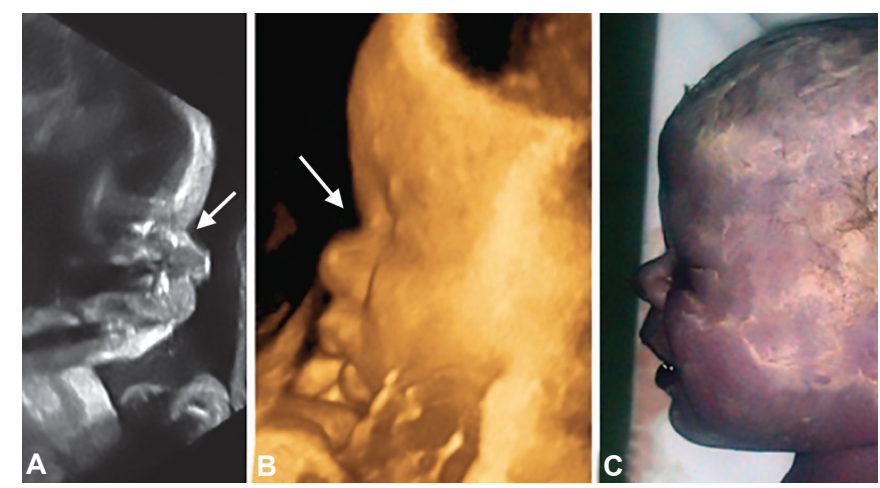

Figs 37A to C: Typical face of achondroplasia

craniosynostosis. ${ }^{59-61}$ Minimum mode surface rendering can show details such as ear lobes which are markers of urinary system pathology. The study of the fetal face is now the less important and significant field of the threedimensional study of the cephalic extreme. The so called "granny" effect that is achieve pleasing images of the fetal face to take home is the playful part of the use of ultrasound that has always existed, even when we were only able to hear the heartbeat. The facial dimorphisms are relevant in certain diseases such as achondroplasia (Fig 37) and in many syndromes (holoprosencephaly, elephant man) where the face is often one of many markers, though often very impressive.Finally today the three-dimensional have bought or better earned its place as a complement or as a significant contribution to the two-dimensional ultrasound diagnostic of malformations.

Internal structures of the brain can be explored in more detail. A correct view of the corpus callosum and pellucid fossa can be obtained in real time by 3-4D with volume contrast imaging in the C-plane. The posterior horns and the vermis can also be explored and their volume calculated. ${ }^{62,63}$ The optic chiasma is easily detected and is 


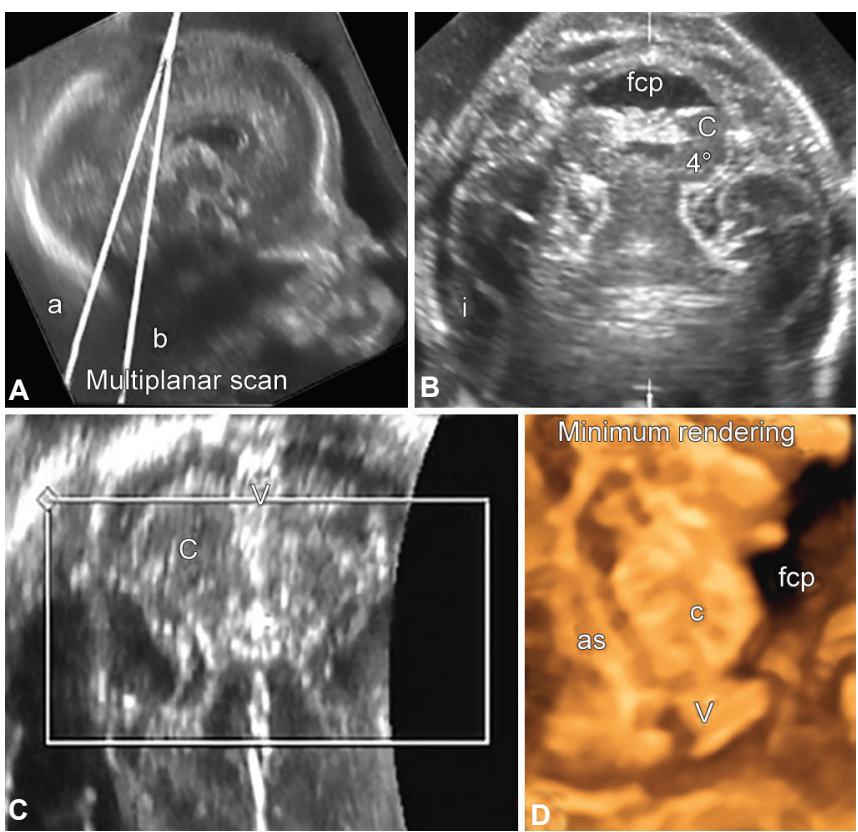

Figs 38A to D: Posterior coronal scan. By 3D multiplanar and minimum rendering we can obtain a view of the cerebellum, fourth ventricle, acqueductus silvii and vermis that is difficult to obtain by $2 \mathrm{D}$

(Abbreviations: as, aqueductus silviani; c, cerebellum; pcf, posterior cranial fossa; i, insula; v, vermis; 4 , fourth ventricle)

an important prognostic factor in cases of anterior brain anomalies. Brain vascularisation provides much material for future study. The Willis circle and the pericallosa and marginal arteries are easily detected in glass-body mode and angio mode of power or colour Doppler, from the first trimester (Figs 38 to 40).

Detailed study of the jaw and oral cavity is another aspect of the head. We have extensively studied the ears, their size, morphology and position (normal or low), prompted by observations of neonatologists who attribute importance to the anatomy of the external ear (Figs 36 and 41 to 51). Neurosonology represents the area in which the three-dimensional really makes the difference enabling the operator to browse inside the brain structures using multiplanar method; the study of the corpus callosum has become a reality in almost all cases, with the ability to detect the partial agenesis, while both ventricles and posterior fossa are now easier to explore.

Inside the cranium is now possible to highlight structure otherwise unimaginable as the optic chiasm or sphenoid bone (Figs $43 \mathrm{H}$ and I), although currently there is a lack of clinical utility but it is representative to signify the depth anatomical study allowed by 3 and 4D. Finally, the study of the palate which today represents a new barrier demolished by ultrasound with a great contribution of the three-dimensional. (Figs 42 to 51)

Renewed interest in the study of primary and secondary palatal morphology has arisen with new ultrasound diagnostic methods in 3 and 4D (64 to 66) and the fact that facial cleft accounts for $13 \%$ of all congenital malformations. ${ }^{67}$ Malformation of the lip and palate may be isolated, associated with other malformations and/or sequences of malformations, associated with chromosome anomalies or with manifestations of a syndrome. Typical facial cleft, including cleft lip, cleft lip/cleft palate and cleft palate, have a prevalence of 9.1/10,000 and 6.4/10,000 births, respectively. Cleft lip accounts for $36 \%$ of all lip and palate malformations and the birth prevalence of isolated orofacial clefts accounts for $61.67 \%$ of total facial clef. ${ }^{68}$ The prevalence is high (about one/1000) and in about two thirds of cases not only involves the lips but also the palate. Unfortunately in 45 to $47 \%$ of cases the defect affects the palate only. ${ }^{69}$ From an epidemiological viewpoint, isolated malformations of the palate are associated with other malformations in about $18 \%$ of cases and with syndromes in $27.2 \%{ }^{70}$ Cleft lip-cleft palate is isolated in $70-79 \%$ of cases and in the other 21 to $29 \%$ it is part of a syndrome or associated with other malformations. ${ }^{71-73}$ Chmait $2006^{74}$ reports 45 cases of cleft lip-cleft palate diagnosed by 2D and 3-4D scan, among which $21.6 \%$ of forms diagnosed as isolated
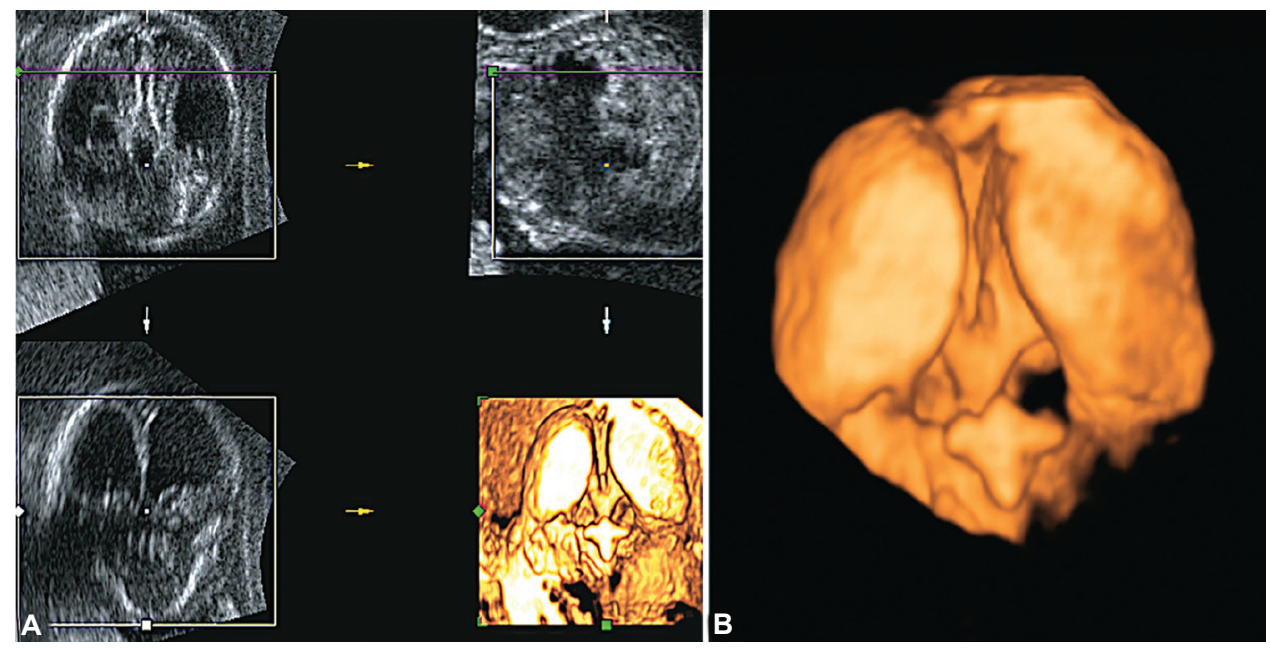

Figs 39A and B: Invert and vocal modes are suitable for determining hydrocephaly 


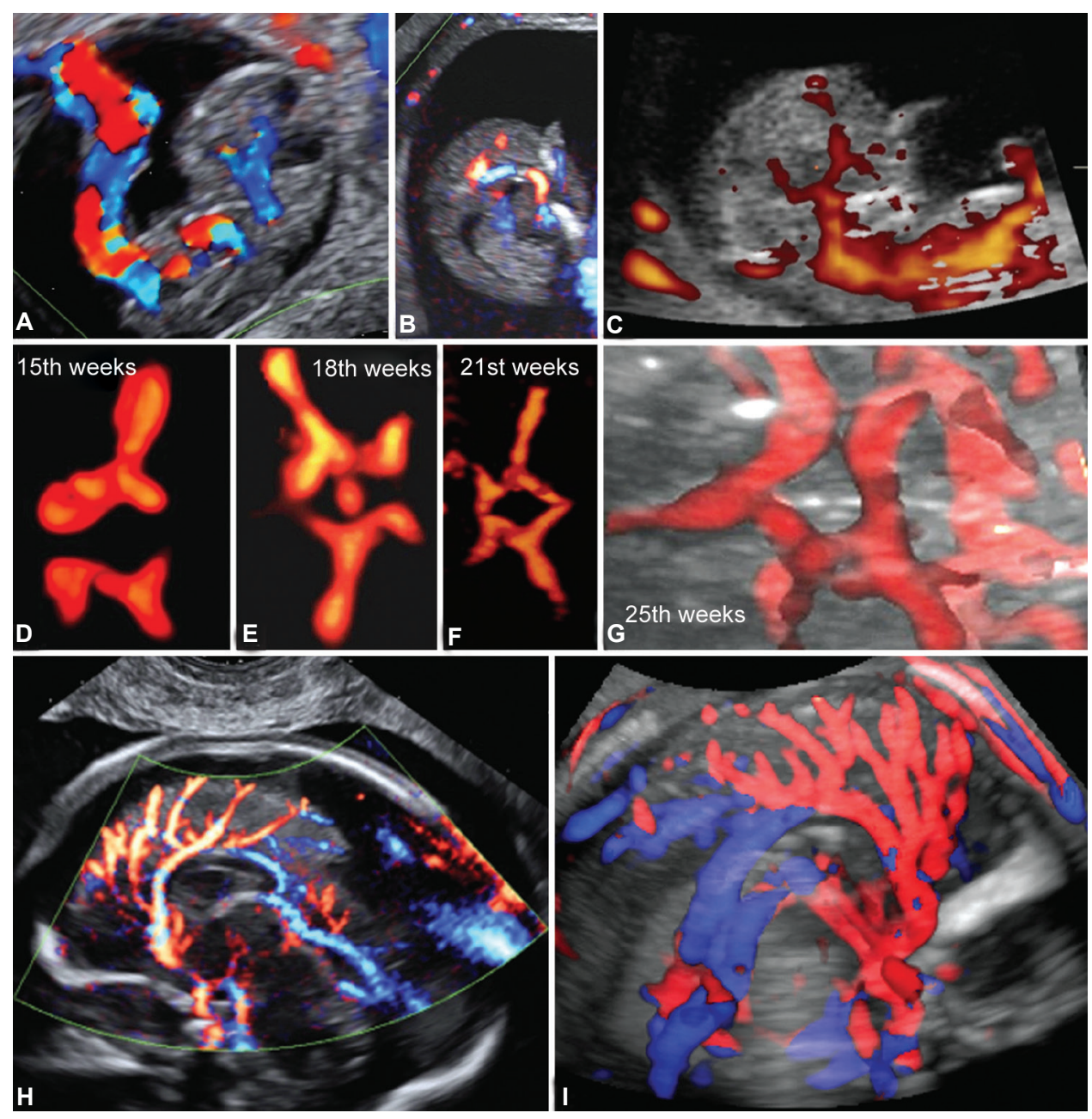

Figs 40A to I: Brain circulation starts in the first trimester of pregnancy. The Willis circle and pericallosa artery are readily viewed by power or color glass-body mode

revealed malformations not detected by ultrasound at follow-up. The report EUROSCAN $2000^{75}$ documents a total ultrasound sensitivity of $27 \%$ for cleft lip-cleft palate, a sensitivity of $17 \%$ for isolated forms and $7 \%$ for isolated cleft palate. Other reports indicate a detection rate of up to $73 \%$ for cleft lip by 2D scan performed after week 20 of pregnancy ultrasound but there are papers which report sensibility for isolated cleft of palate about $0 \% .{ }^{76}$ The prevalence of this malformation and especially the high incidence of associations with other anatomic and genetic malformations has prompted research to improve ultrasound definition of the secondary palate. Usually the amniocentesis for caryotipe should be offered in all cases of cleft lip/palate because of the risk of aneuploidy; also the patients should be counselled that ultrasound occult additional anatomic abnormalities might be present with all clefts. ${ }^{77,78}$

\section{Ultrasonographic detection of the palate}

The primary palate includes the lips and jaw bone to the nose root and is the most easily detected part of the anatomy by 2D scan (Figs 52 and 53). Indeed many sustain
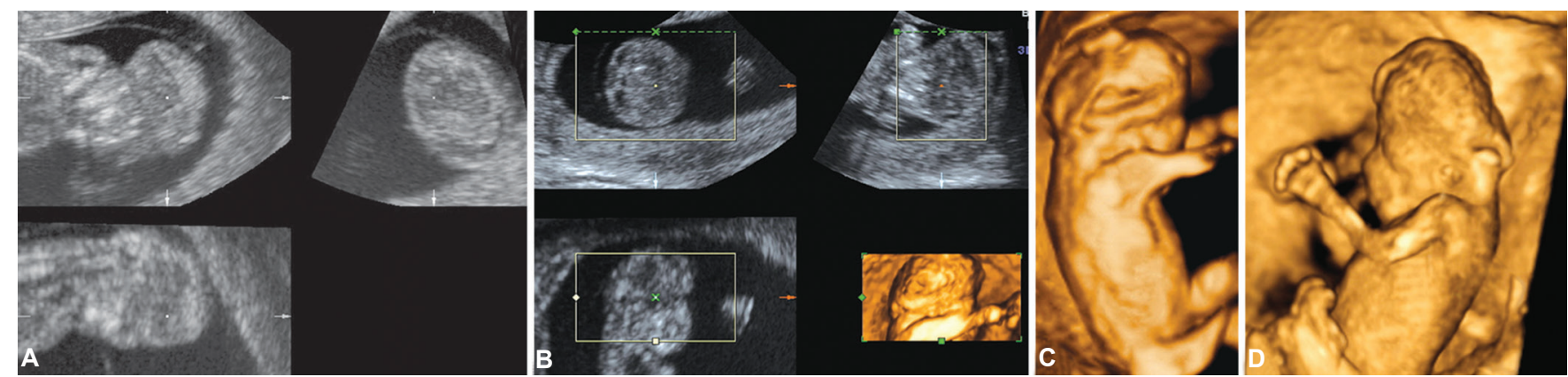

Figs $41 \mathrm{~A}$ to $\mathrm{D}$ : Anencephaly at week 11 . The image shows the typical face and the absent skull 


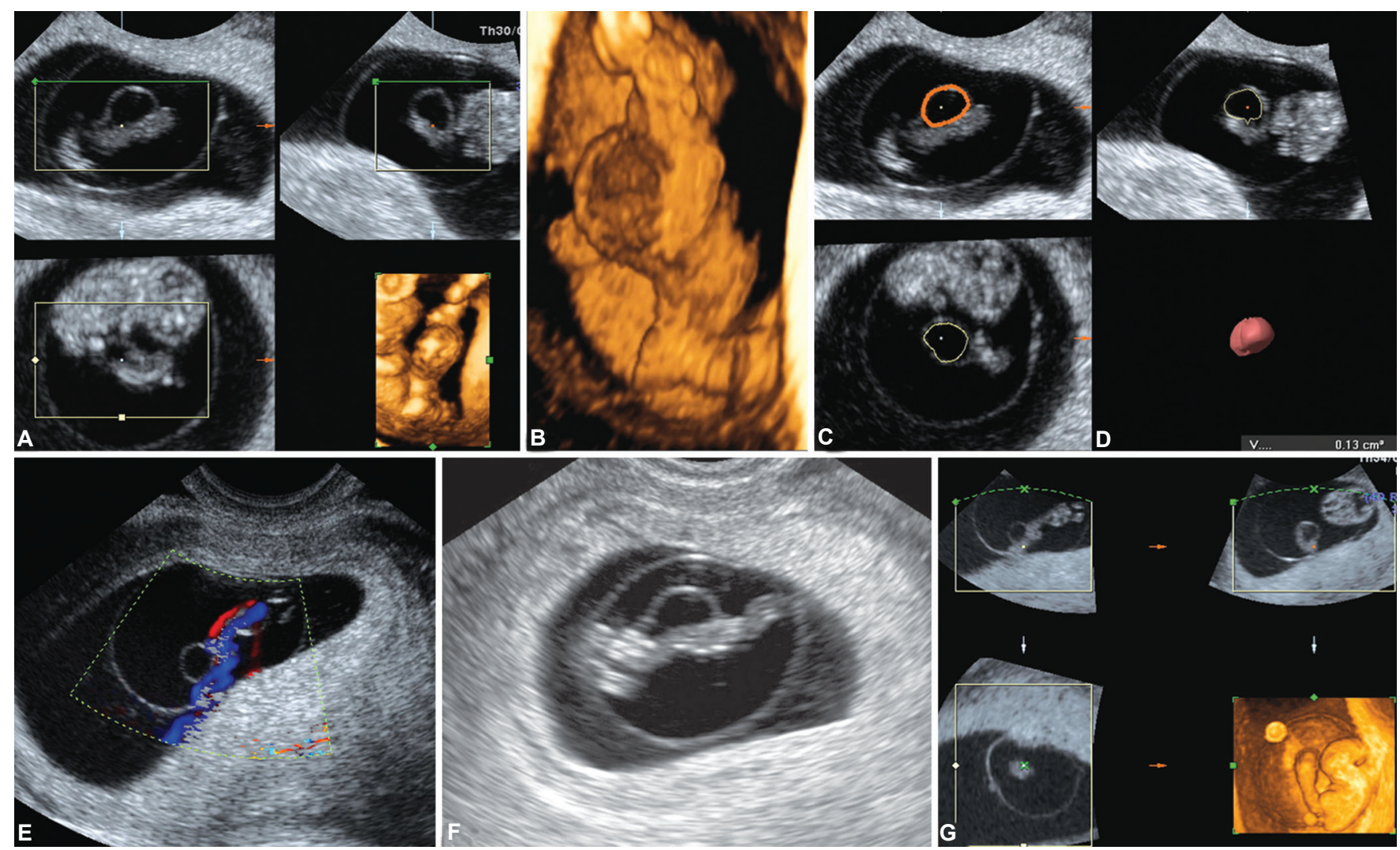

Figs 42A to F: Umbilical cord cyst at week 9 . This is a transient soft marker of aneuploidy (about $25 \%$ sensitivity-present in about $5 \%$ of pregnancies)

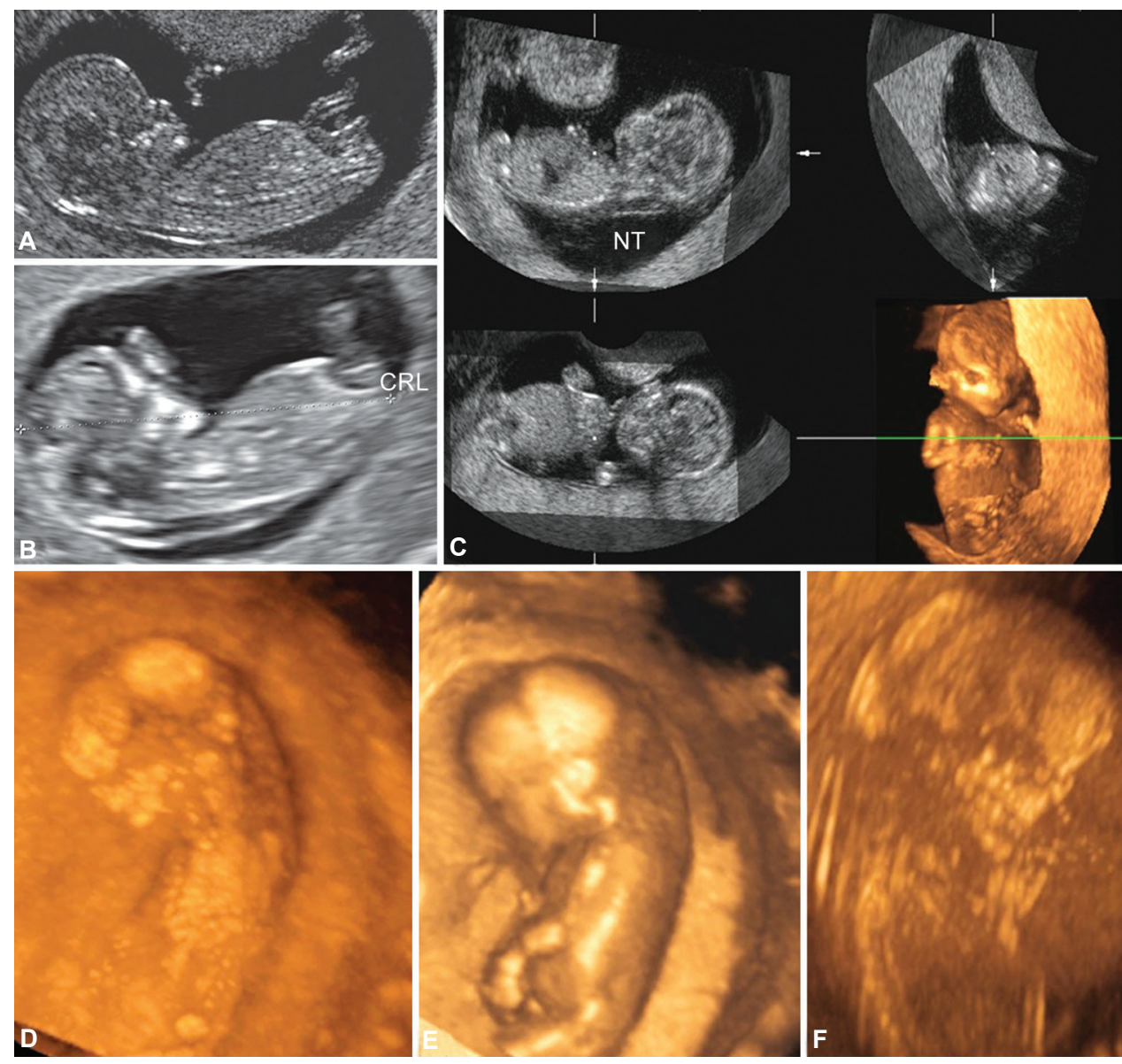

Figs 43A to F: The difference between $2 \mathrm{D}$ and $3 \mathrm{D}$ for detection of nuchal translucency is usual minor: $2 \mathrm{D}$ is better for routine scan; $3 \mathrm{D}$ sometimes has advantages when the fetus is in an inappropriate position 

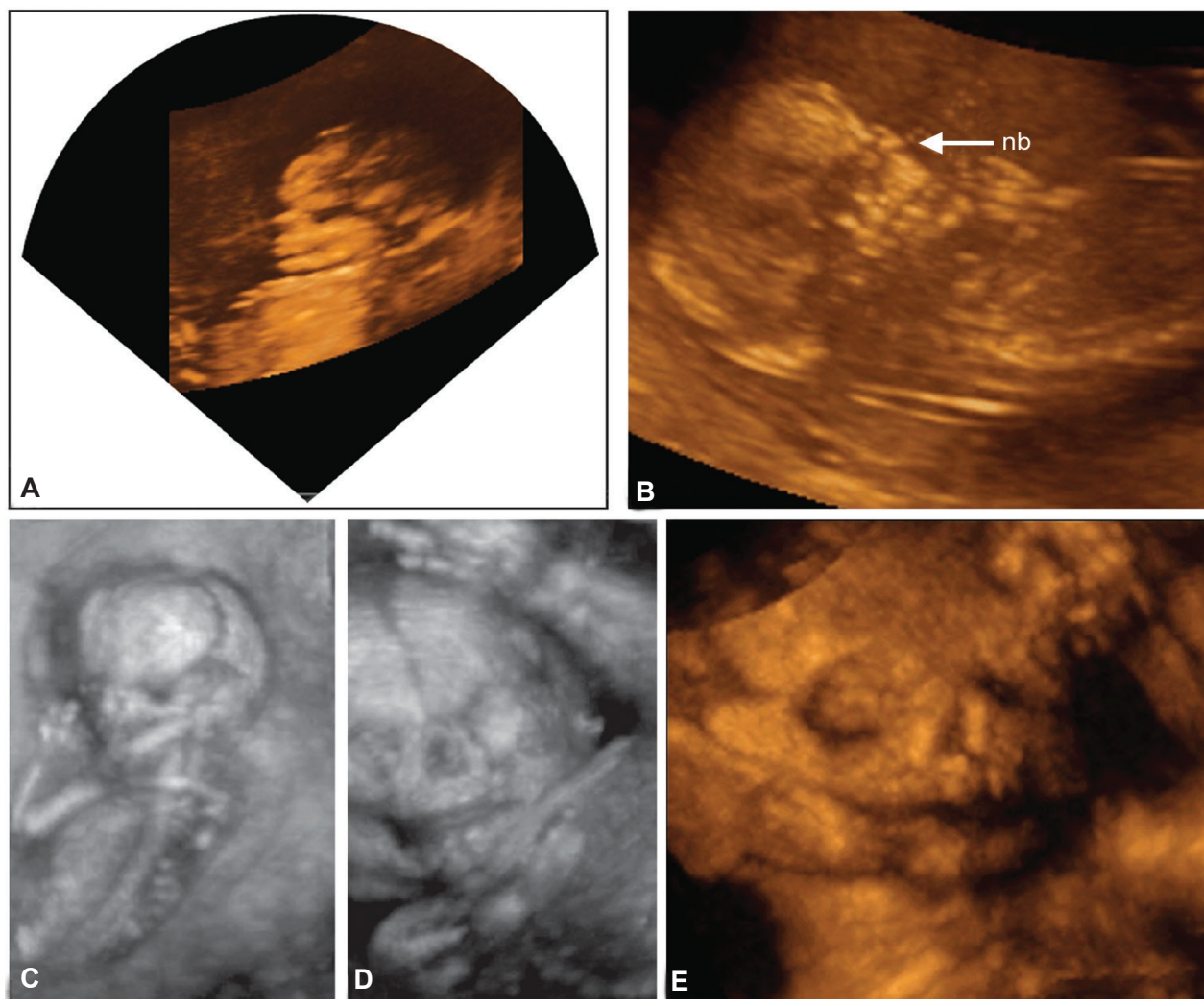

Figs 44A to E: Weeks 13 and 20. It is easy to find the nasal bone and to detect both bones by 3D in the first and second trimesters; it is more difficult by 2D. The pictures are at 13th and 20th weeks (Abbreviation: nb; nasal bone)
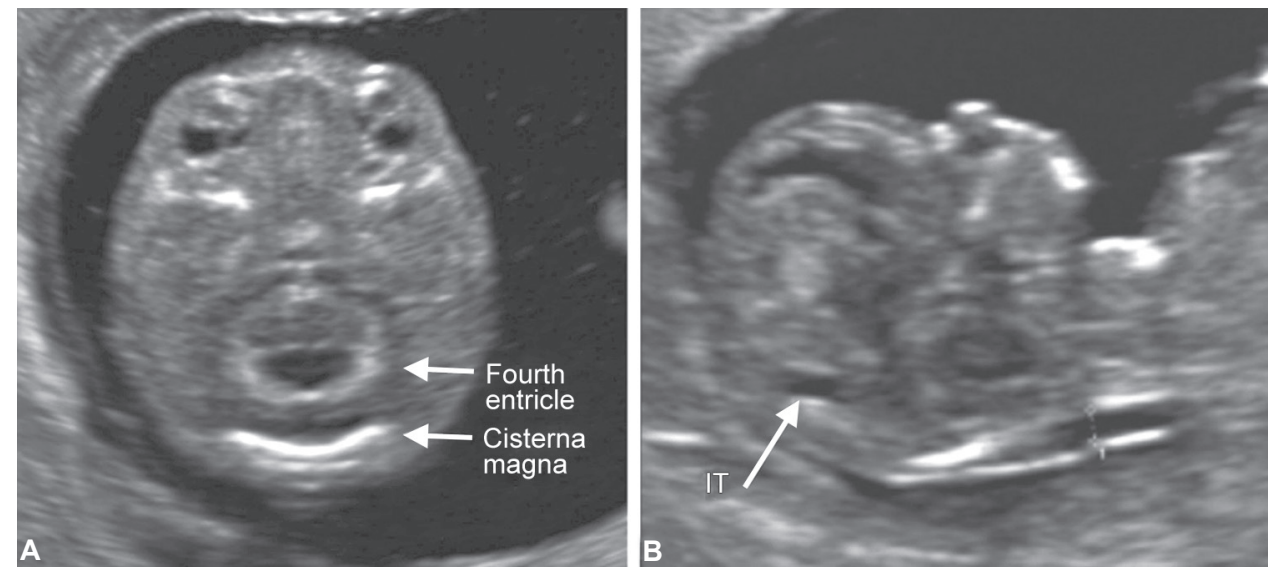

Figs 45A and B: Intracranial translucency 2D and 3D. It is easy with a correct scan to find the IT between two hyperechoic lines

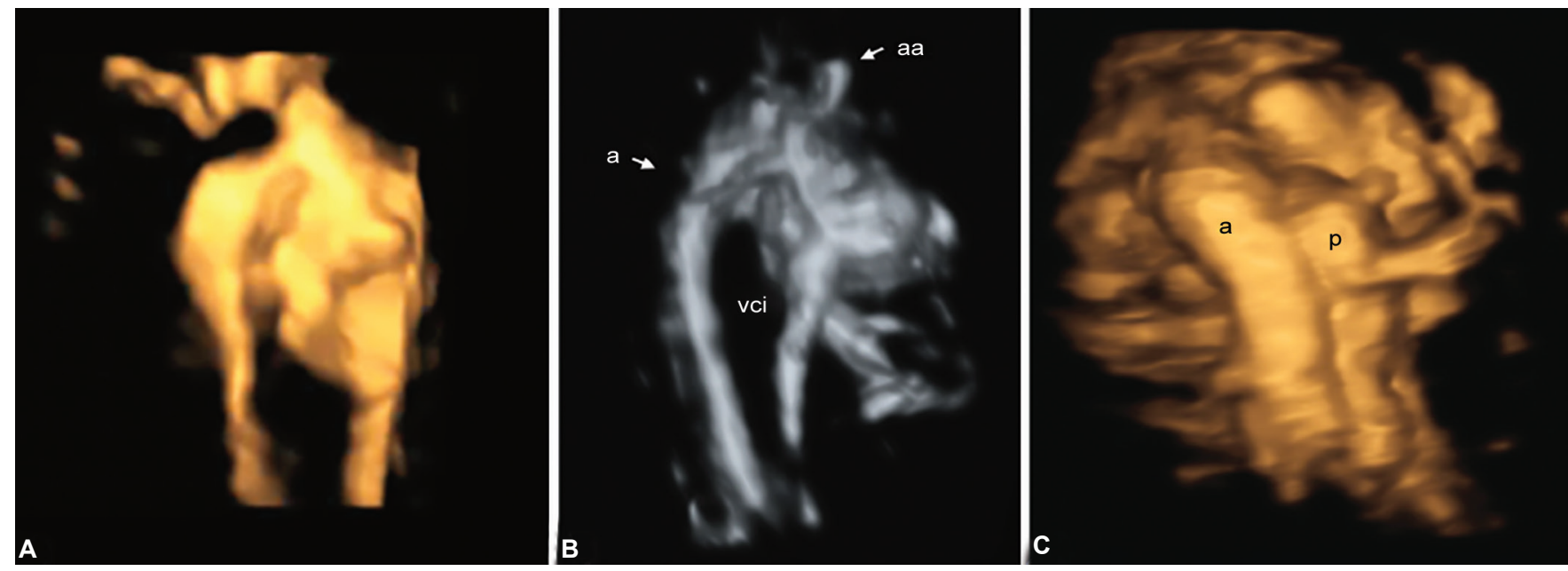

Figs 46A to C: An example of great vessels transposition. The b-flow is not an easy technique but improves the heart defects about pulmonary veins. (Abbreviations: a, aorta; p, pulmonary artery; vci, inferior vena cava; aa, anonymous artery) 

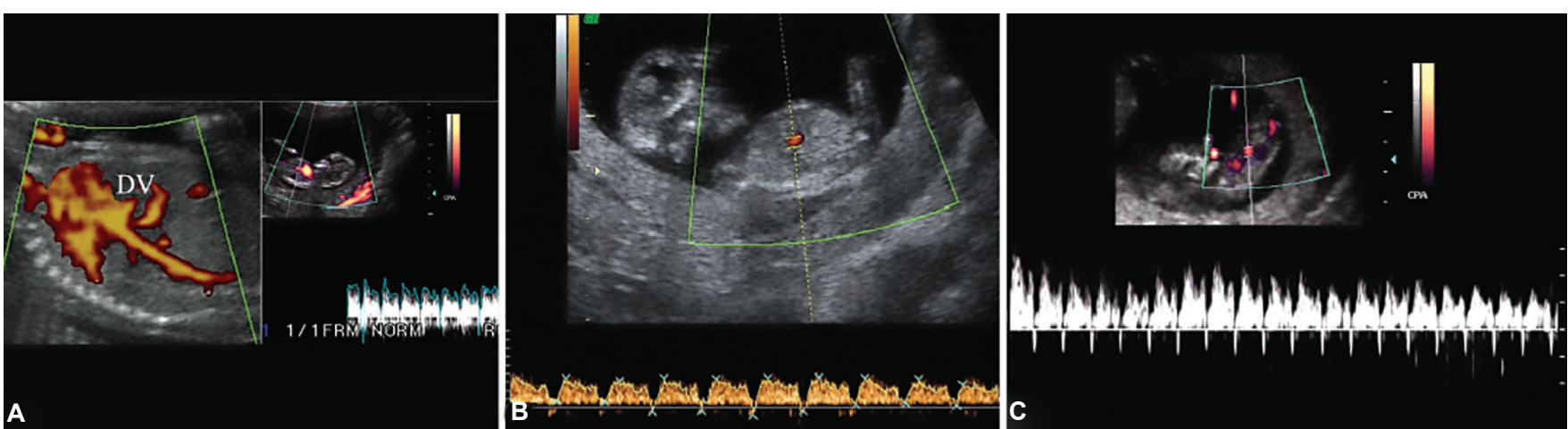

Figs 47A to C: Ultrasound flow imaging of the ductus venosus is only feasible by 2D, but 3D provides good images of the vessel. The images show normal A wave in ductus venosus in first trimester and a case of reversed flow

(Abbreviation: DV; ductus venosus)

that it is worthwhile visualising the lips, jaw bone and nose root with an oblique coronal scan, scrolling upwards during the routine second trimester scan and it is evident to users of 3D that this suggestion is almost superfluous when a scan of the foetal face is part of the routine (Fig. 54). The secondary palate consists of a hard palate, which runs posterior and horizontal to the incisive foramen and soft palate or velum, which curves downwards and backwards from the posterior aspect of the hard palate and ends in the uvula. In the foetus the hard/soft palate is 2.1 and the soft palate has the similar thickness (Figs 15 to 22). Usually the cleft of the secondary palate is always midline and results from failure of the palatine processes to elevate and grow (Figs 16 to 18 and 20). Cleft of the secondary palate starts from uvula and soft palate, but it is possible the cleft of soft palate with an intact hard palate $^{79}$ (Fig. 19). The severe shadowing of the maxilla made difficult but not impossible the visualization and the diagnosis of clefts of the secondary palate. Shereret et al. ${ }^{80}$ says that visualization of the secondary palate is not difficult by axial plane 2D scan, but he doesn't report any cases of defects of secondary palate. In this case the new volumetric probe 3D multiplanar and surface rendering offers greater possibilities of study of the normality
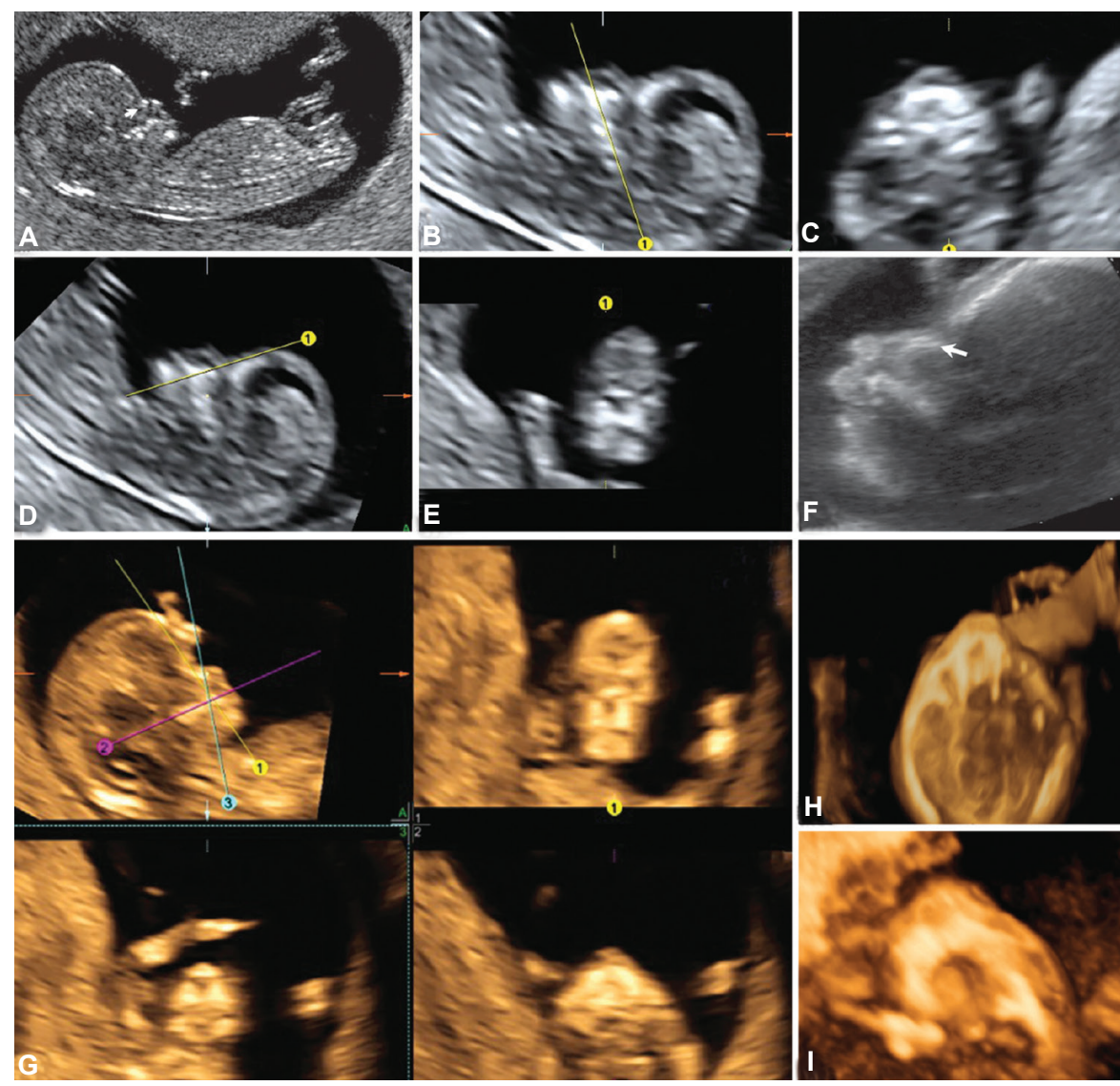

Figs 48A to I: It is possible to control the normal secondary palate in the first trimester of pregnancy by Faure technique 3D (delta sign ( $D$ and $E$ ) by rendering surface or $(C)$ by omniview) or by retronasal triangle ( $A$ to $C$ ) by $2 \mathrm{D}$ or by omniview) 

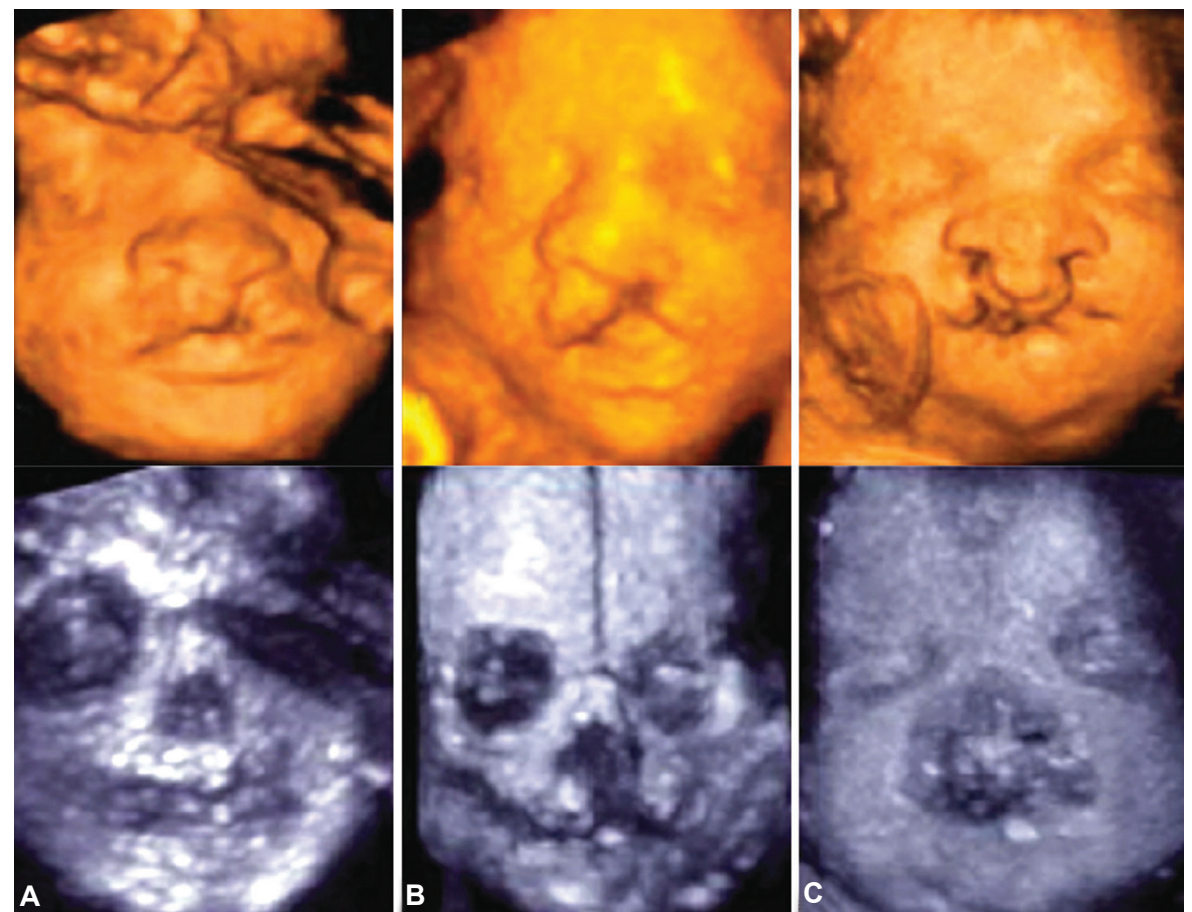

Figs 49A to C: Maximum and minimum rendering in three cases of $\mathrm{l}-\mathrm{p}$ cleft. It is easy to understand the extension of the cleft

and of diagnosis of the cleft of the primary and secondary palate. ${ }^{81-83}$ By $3 \mathrm{D}$ it is possible to see the alveolus and maxilla by axial scan and secondary palate by coronal scan by scrolling front-to-back in coronal plane. But with this method there is the problem of the maxillary shadow. Campbell ${ }^{84}$ overcame this problem by rotating the face through $180^{\circ}$ and scrolling from back-to-front. This technique, described as 'reverse face view' eliminate the shadowing of the maxilla, but it offers the possibility to have a good vision of the hard palate but not of the soft palate. Platt et al.$^{85}$ found a different technique to see also the soft palate by axial 3D plane (multiplanar and surface rendering) with inverted picture to avoid the shadowing of the maxilla and using a little acoustic box scrolling from chin to nose (flipped-face view'). Uses a sagittal scan and by this technique the mandibula, the tongue, the maxilla,
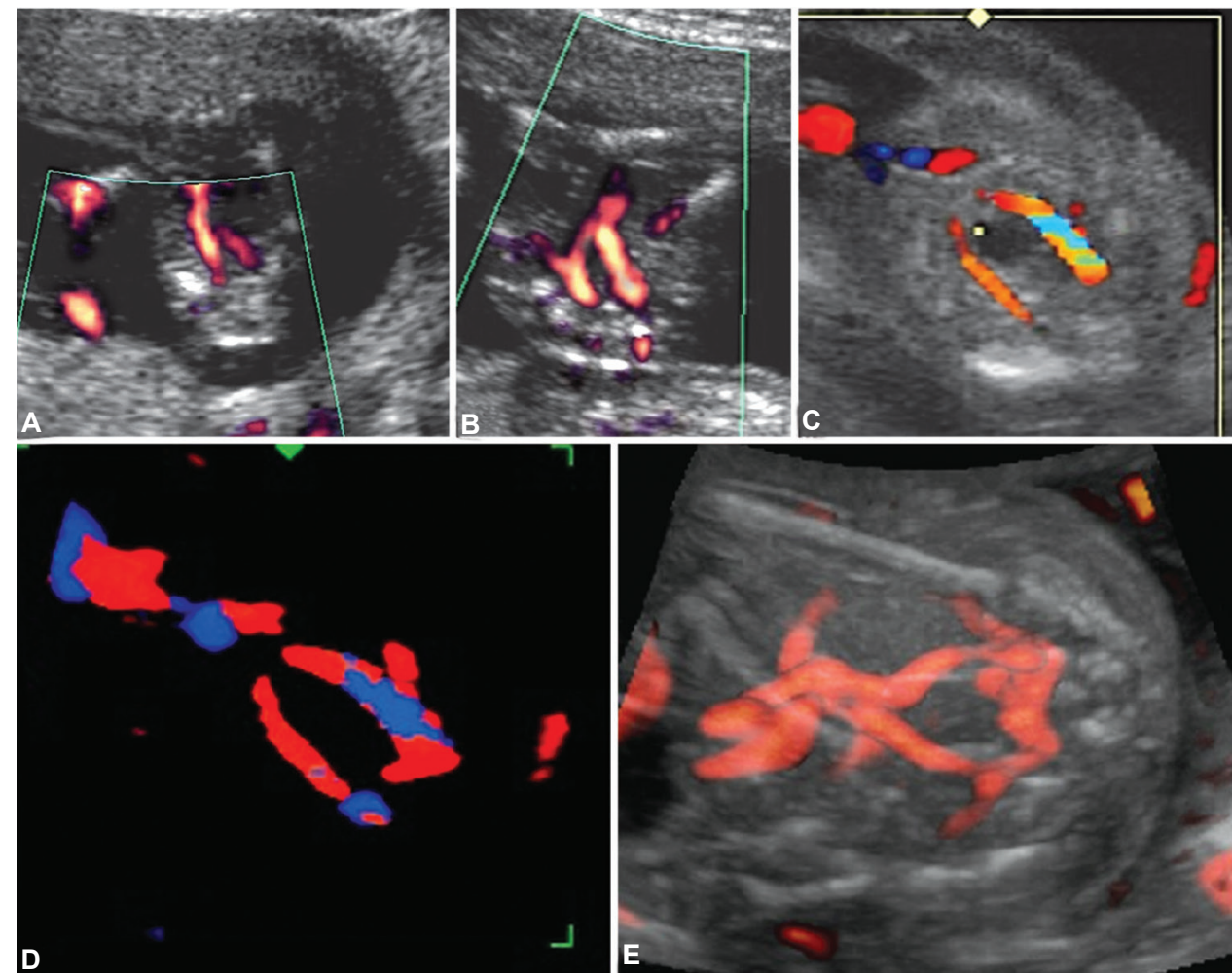

Figs 50A to E: In the first and second trimesters, it is easy to find both umbilical arteries at bladder level; 3D color, power and glass body modes provide more realistic images 


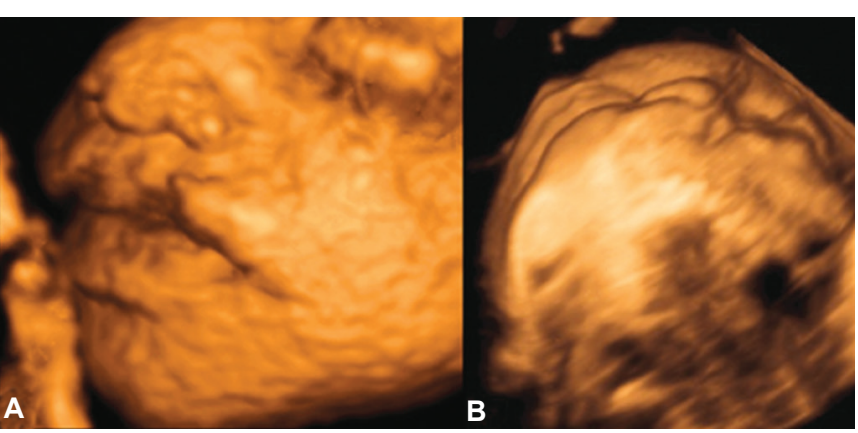

Figs 51A and B: Cleft-lip and cleft alveolar ridge by surface rendering front face

the alveolar ridge, the secondary complete palate are systematically seen and offers a good mode to diagnosis the clefts of the primary and secondary palate. Faure et al. ${ }^{86}$ propose the same technique of Platt by coronal scan, because were able to obtain the view of the palate in all 100 low-risk cases, all with normal anatomy after delivery, from 17 to 23 weeks. Pilu and Segata ${ }^{87}$ describe a new multiplanar approach to study the secondary palate using TUI (Tomography Ultrasound Imaging); to avoid the shadowing of the alveolar ridge the authors used a scan with an angle of $45^{\circ}$ and obtained a satisfactory view in 10 of 15 cases between 19 to 28 weeks. Now, then, we have many possibility to study systematically the primary and the secondary palate by 2D, but with greater possibilities by $3 D_{;}^{, 88}$ it is reasonable says that gold standard is to have experience with all techniques, above all in cases of doubt or of diagnosis of facial cleft to define exactly the limit of lesion. By axial 3D plane (sagittal or coronal scan) Campbell ${ }^{89}$ propose a screening of secondary palate in the first trimester; in fact it is easy from 11 to 14 weeks to see the secondary palate (delta sign) but, if the screening is effective in diagnosing orofacial clefting will require further study. (Delta sign) (Figs 55 and 56). The scan in the second trimester, week 19-22, is associated with a greater possibility of detecting the primary and secondary palate and of diagnosis of facial clefts (Figs 57 to 61).
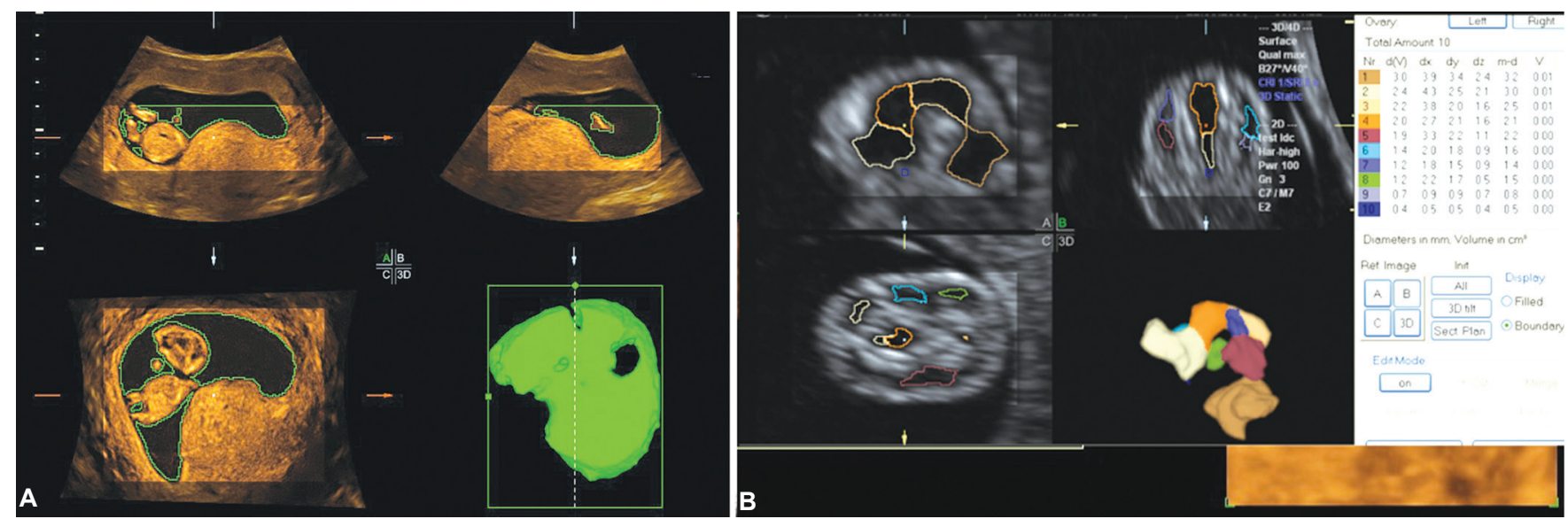

Figs 52A and B: The control of volume of amniotic fluid in the first trimester or the dimension of ventricles is possible by sonoAVC

\section{Secondary Palate}

The scheme of Berkowitz (Fig. 62) illustrates that the uvula is always involved in cleft palate and could simplify detection, but in practice it is difficult to detect this small anatomical part with 2D and 3-4D.

\section{Two Dimensional}

Two-dimensional scans can be used to study the hard and soft parts of the secondary palate and detect the uvula (Fig. 20). However it is necessary that the fetal head be in a favourable position, possibly with the lips slightly apart. Today (2010 Whilhelm) a new marker "the equal sign" offers a new easy possibility to detect or suspect an isolated palate cleft. In axial scan (better than coronal, inusual and difficult), with the same scan to measure the BPD with a little inclination, is relatively easy find the farinx like an anechoic round area with a double signs of the uvula foto. In case of the bifidus uvula it is important to study the secondary palate and the 3D (min-max rendering, Faure method or others, ominiview) became a beautiful tool to investigate.

\section{Three and Four Dimensional}

Three-dimensional scans have greater possibilities because a volume can be saved and examined later in an infinite number of scanning planes. Various methods have been proposed:

- Axial surface rendering plane with a small box ("flipped-face view") and inverted scan to avoid maxillary shadow (scrolling upwards) by sagittal or coronal scan (Figs 55 to 61 and 63 to 70)

- Coronal surface rendering plane, reverse-face (scrolling forwards) or front face (scrolling backwards) scan (Figs 68 and 69)

TUI tomography ultrasound imaging sagittal scan with angle of $45^{\circ}$ (Fig. 70). 

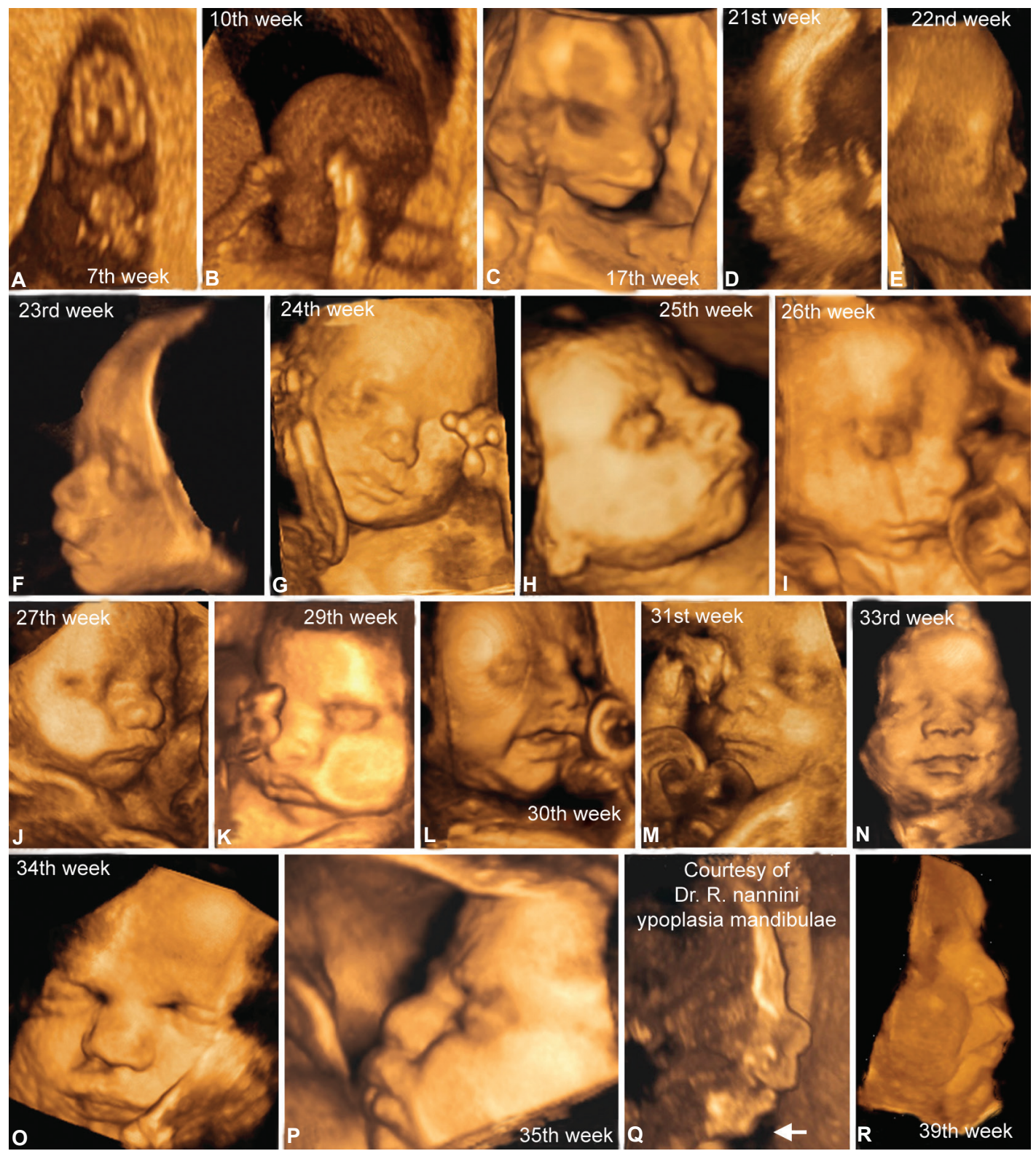

Figs 53A to R: The fetal face acquires human features during gestation. The face of the embryo is quite unattractive; whereas in the second trimester, it becomes softer and more pleasant and remains thus into the postnatal period. Facial modeling is related to the formation of facial musculature, fat and the thick consistency of skin due to soaking. 3-4D provides completely realistic images, especially with high quality instruments and experienced operators. It is therefore relatively easy to assess facial symmetry and reliably recognize facial dysmorphisms (e.g. mandibular hypoplasia), which may be of genetic disorders and to diagnose cleft lip or palate

Ultrasound technicians will be increasingly called percentage of malformations, syndromes and chromoupon to check the integrity of the secondary palate due to some anomalies, associated especially with cleft palate. the high prevalence of facial defects at birth and the high Three-dimensional techniques offer ways of achieving
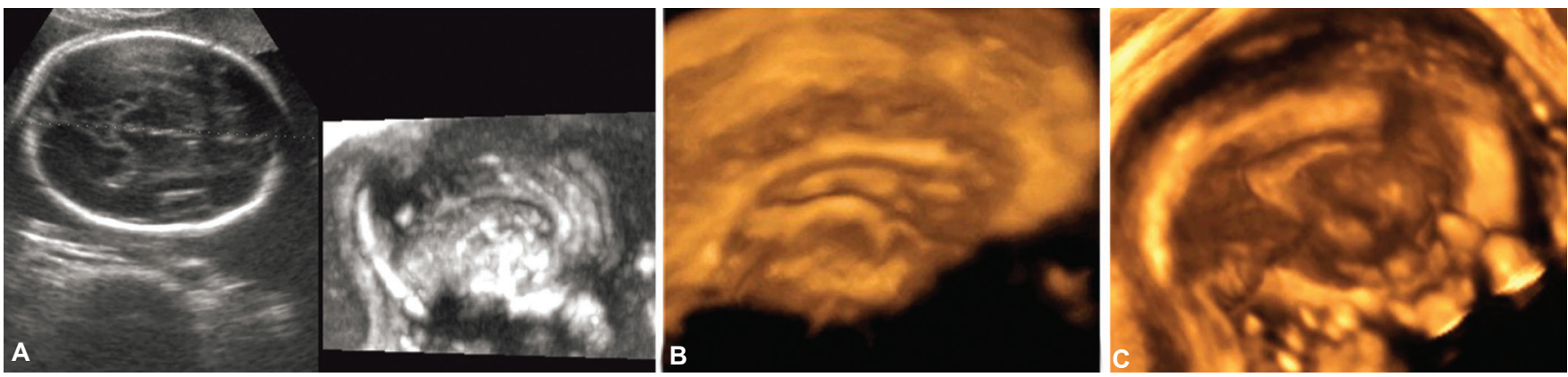

Figs 54A to C: Examination of the corpus callosum is easy and quick by 3D and volume contrast imaging in c-plane; by $2 \mathrm{D}$ it is very difficult 

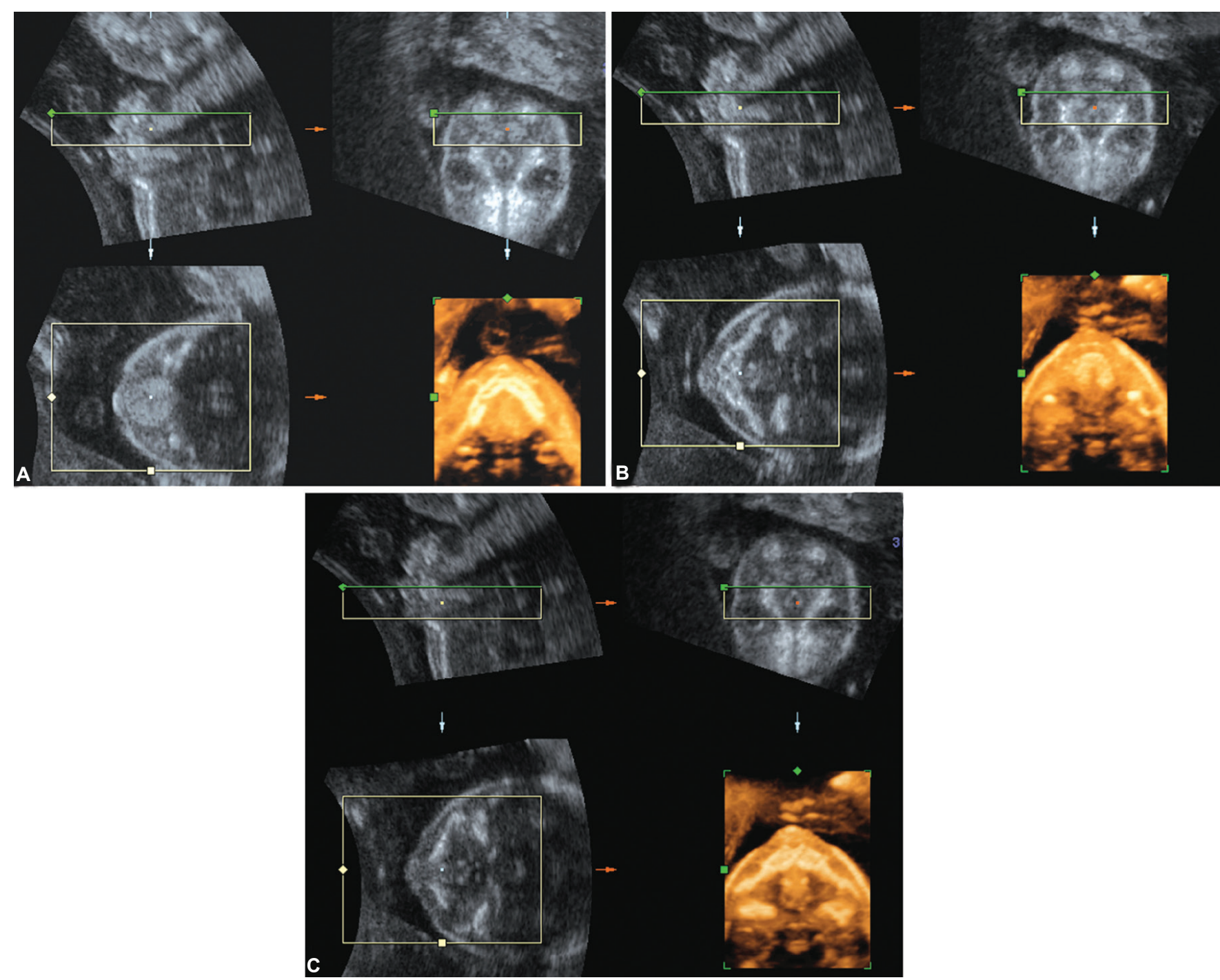

Figs 55A to C: Secondary palate by 3D multiplanar and surface rendering; axial plane (flipped view face) with sagittal inverted (to avoid the maxillary shadow) scan; scrolling up-down: (A) The mandible; (B) The tongue; (C) The maxilla

this. Among those described above, the 3D axial plane is the fastest and easiest to apply, and has been reported to detect the secondary palate in almost all cases in 2 to 3 minutes when echographic conditions do not pose an impediment. Variable fetal position in relation to maternal fat is the only serious obstacle to its correct detection. Today it would seem reasonable to propose study of the hard and soft palate only in cases with suspected or

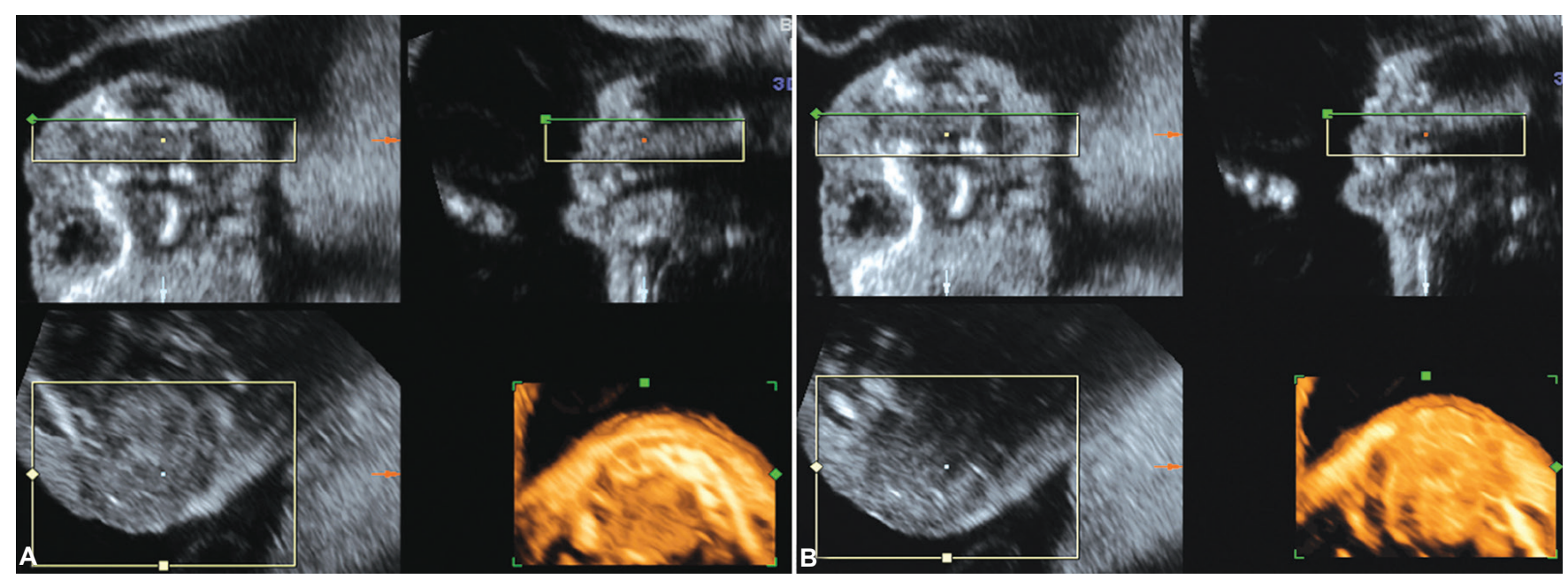

Figs 56A and B: Secondary palate by 3D multiplanar and surface rendering: axial plane with coronal inverted (to avoid the maxillary shadow) scan; scrolling up-down: (A) Mandibular; (B) Tongue and maxillary bone 


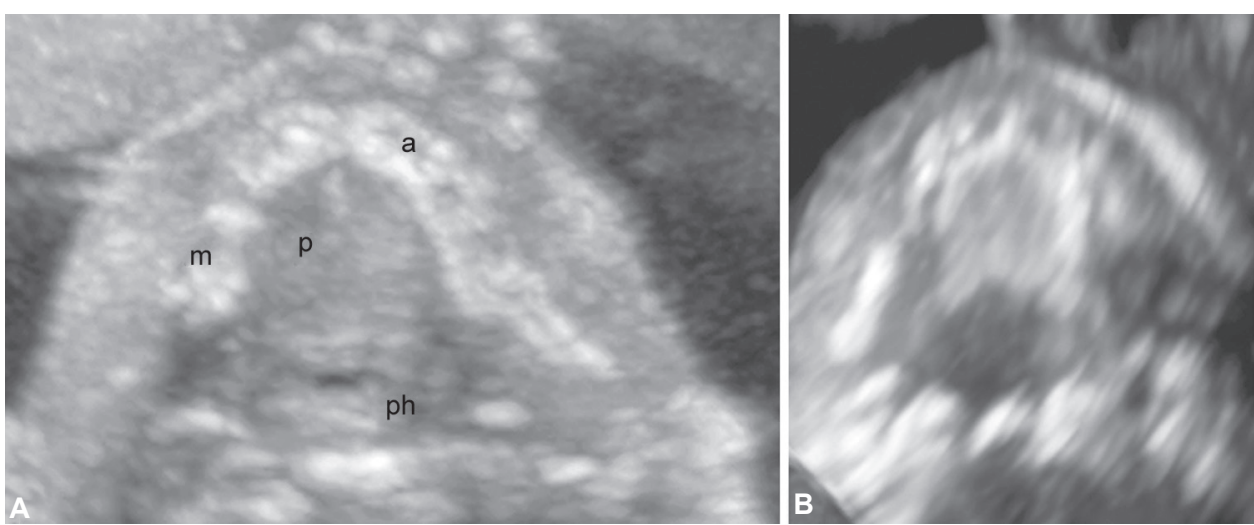

Figs 57A and B: Secondary palate by axial 3D plane (A) Maximum mode and (B) Minimum mode
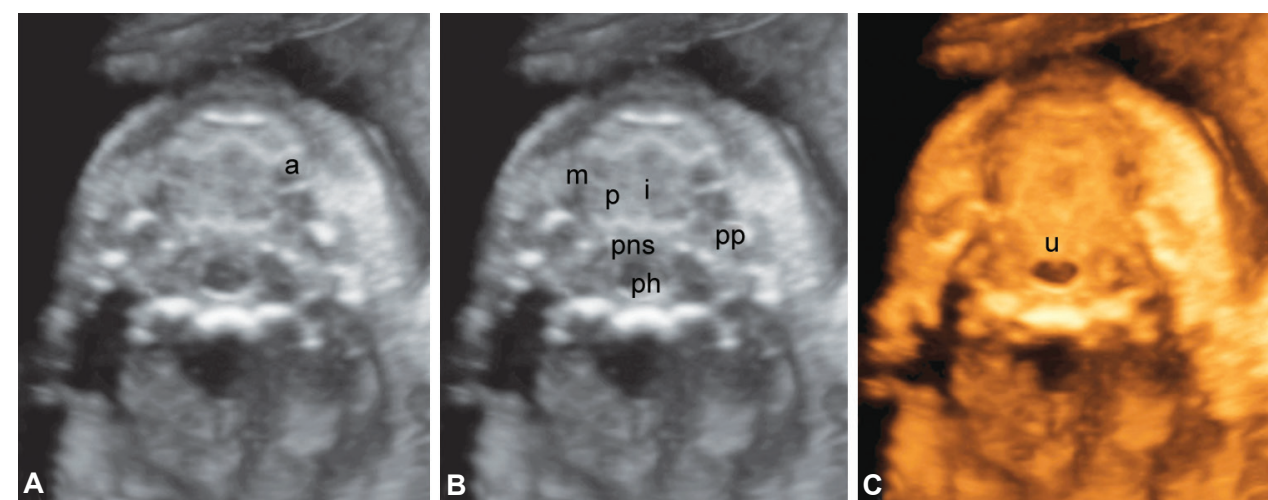

Figs 58A to C: Secondary palate by 3D plane minimum mode without and with captions

(Abbreviations: a, alveolar ridge; $m$, maxilla; i, interpalatal suture; $p$, palatine process; pns, posterior nasal spine; pp, pterygoid process; u, uvula)
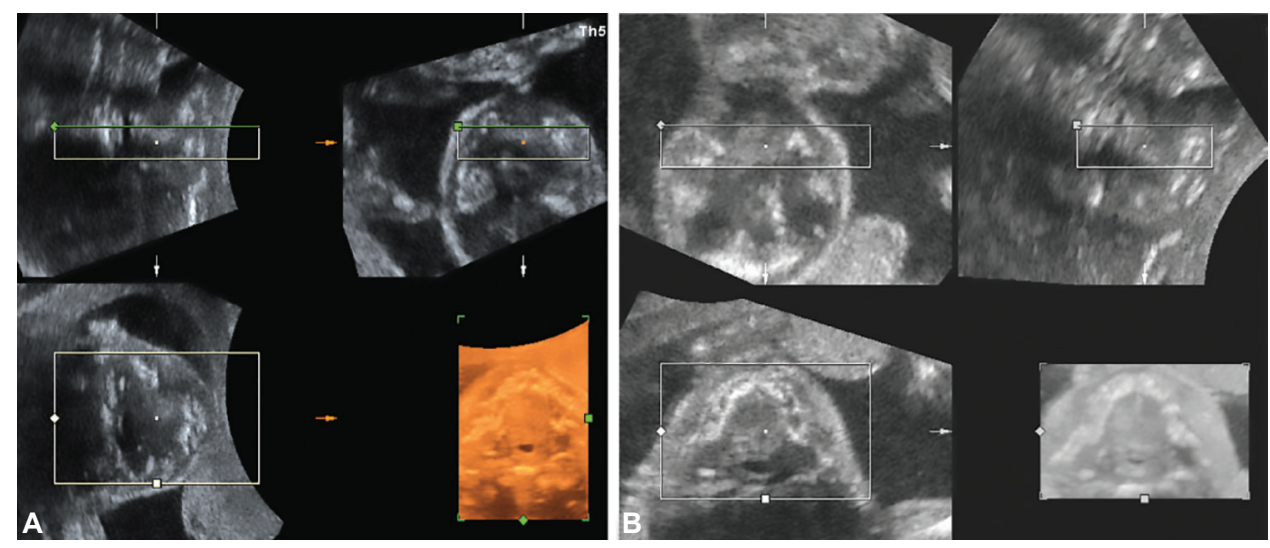

Figs 59A and B: By axial 3D plane, there is no difference between $(A)$ sagittal and $(B)$ coronal scan to see the secondary palate

confirmed diagnosis of facial clefting and in cases with a positive family history or after non visualization of the "equals sign" in 2D (Figs 71 to 76). ${ }^{90}$

\section{Chest}

The Chest contains the heart, an organ fundamental for human life. Study of the heart, efflux and the circulatory system ${ }^{91}$ has exploited 3-4D technology and STIC mode (spatio-temporal imaging correlation), which being in four dimensions, cannot be illustrated in an atlas. However, fixed, invert, power, colour and Doppler 3D images and study in real time by tomography ultrasound imaging (TUI) are striking, interesting, and considerably improve diagnostic capacity. At this time the b-flow is the alone possibility to study the vein efflux with low flow and can also became complementary to STIC in heart pathology; but it is not easy to learn the b-flow. Lung volume can be measured by the VOCAL system ${ }^{92,93}$ improving the prognosis and outcome of fetuses with diaphragm hernia, and is expected to replace thickness as an indication for therapy in utero. With special experience it is possible to correctly visualise the course and morphology of the esophagus. The volume of the thymus, an important foetal organ, can be assessed. The diaphragm is quite evident, reducing diagnostic doubts about hernia. 

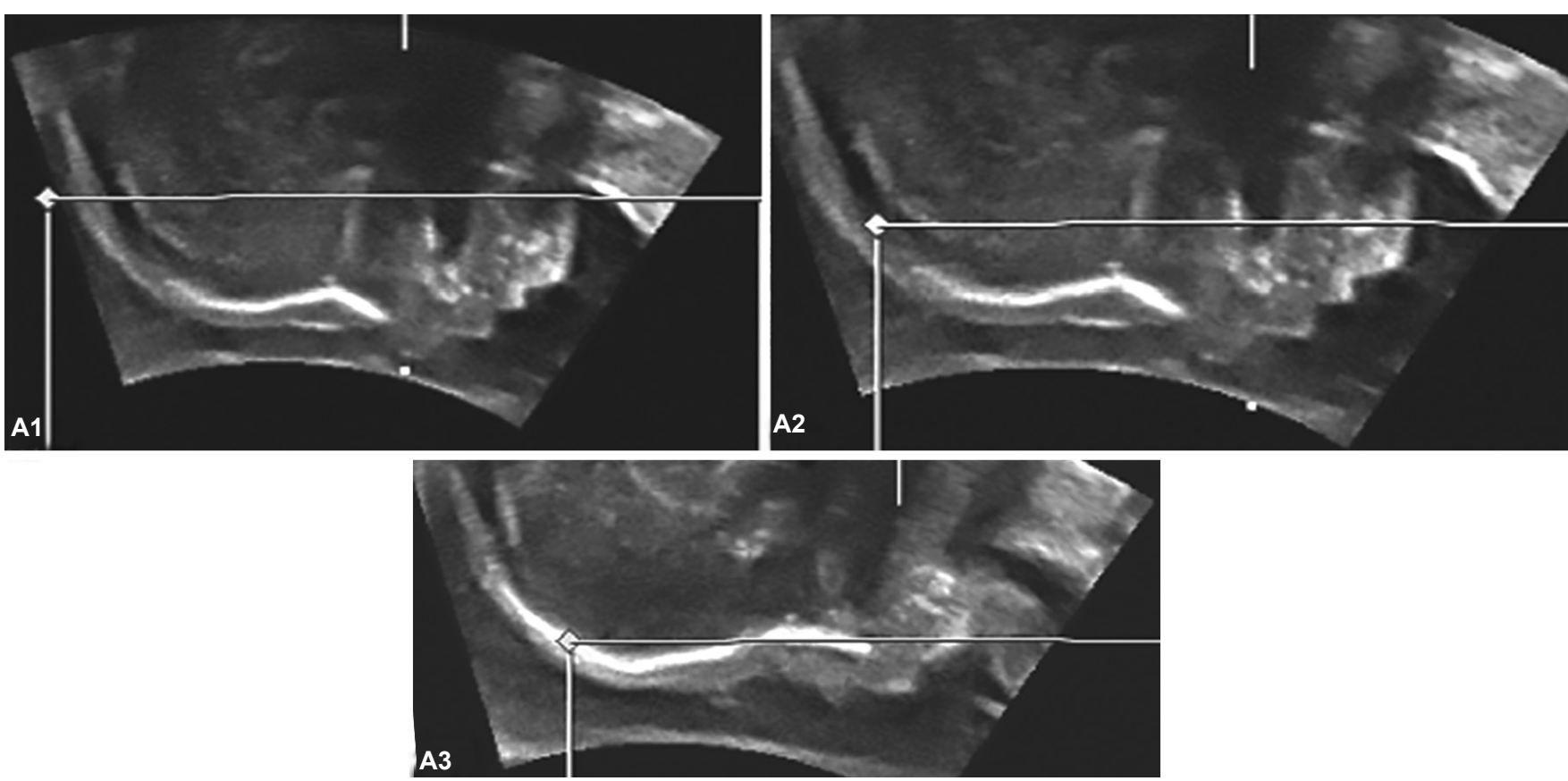

Figs 60 A1 to A3: Secondary palate by coronal 3D plane multiplanar and surface rendering; scrolling forwards (reverse face); A1-B1; A2-B2; A3-B3. The arrow points at hard palate
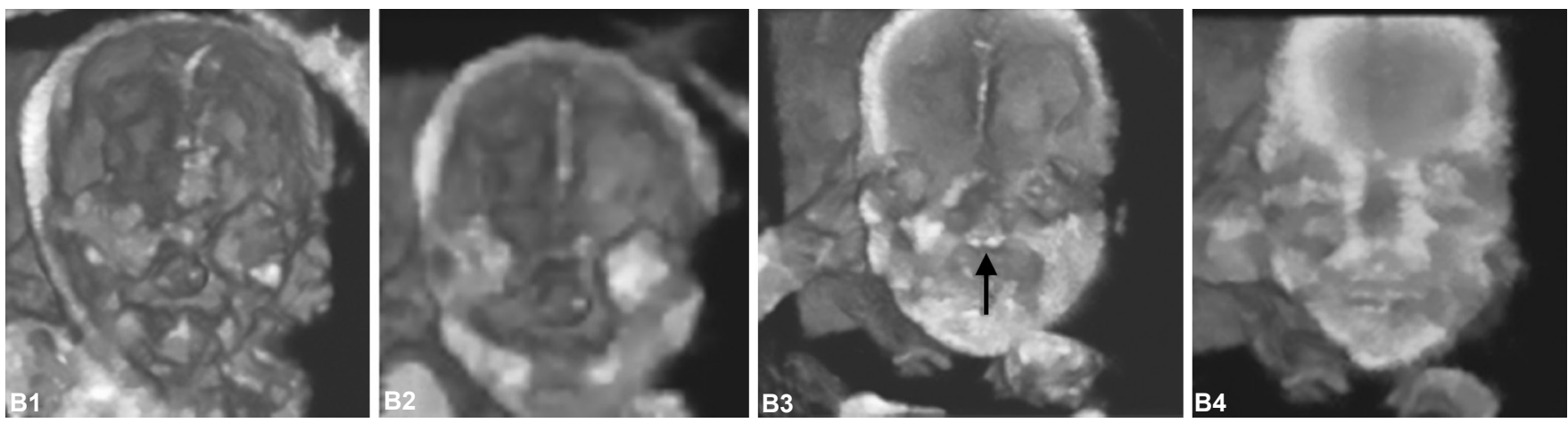

Figs 61 B1 to B4: Secondary palate by coronal 3D plane multiplanar and surface rendering; scrolling forwards (reverse face); A1-B1; A2-B2; A3-B3; A4-B4. The arrow points at hard palate

The gallbladder is also easily visualised (Figs 77 and 78).

\section{Abdomen}

Visualisation of the viscera and stomach are less exciting than the face, though the vascularisation of the liver is striking. ${ }^{94}$ Regarding the urinary system, it is easier to study the renal arteries and parenchyma vessels, and kidney volume is a better index of development than traditional biometry.

\section{Neural Tube and Limbs}

Volume acquisition is one of the most important technological achievements of the last decade in diagnostic ultrasound and offers a good possibility to study the spine. In particular there is the multi-planar which gives the possibility to navigate within a volumetric space through infinite reproducible imaging planes (including the crown), in real time. There is also rendering which

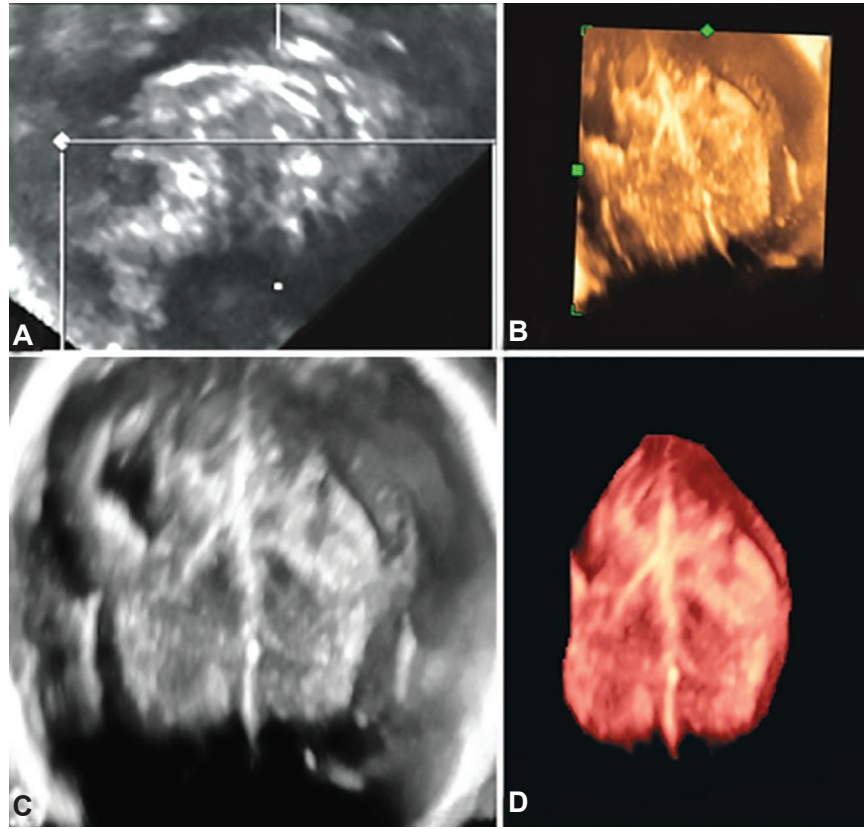

Figs $62 A$ to D: Exploration of the brain by 3D multiplanar mode offers a splendid view of the optic chiasma, important in cases of partial agenesis of the corpus callosum 

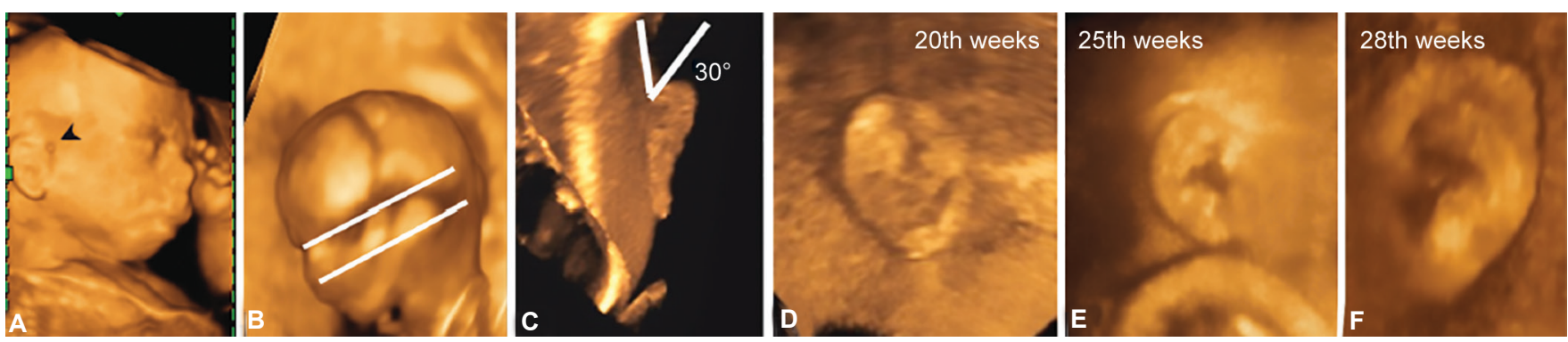

Figs 63A to F: By 3D it is possible to detect very small appendix auricularis (an important marker for many other malformations); ear position is important, because low placement or a helix more than 30 degrees out of the skull are a marker of aneuploidy. External ear morphology changes during gestation: until week 20 it is a ring; final morphology is only achieved at about week 29
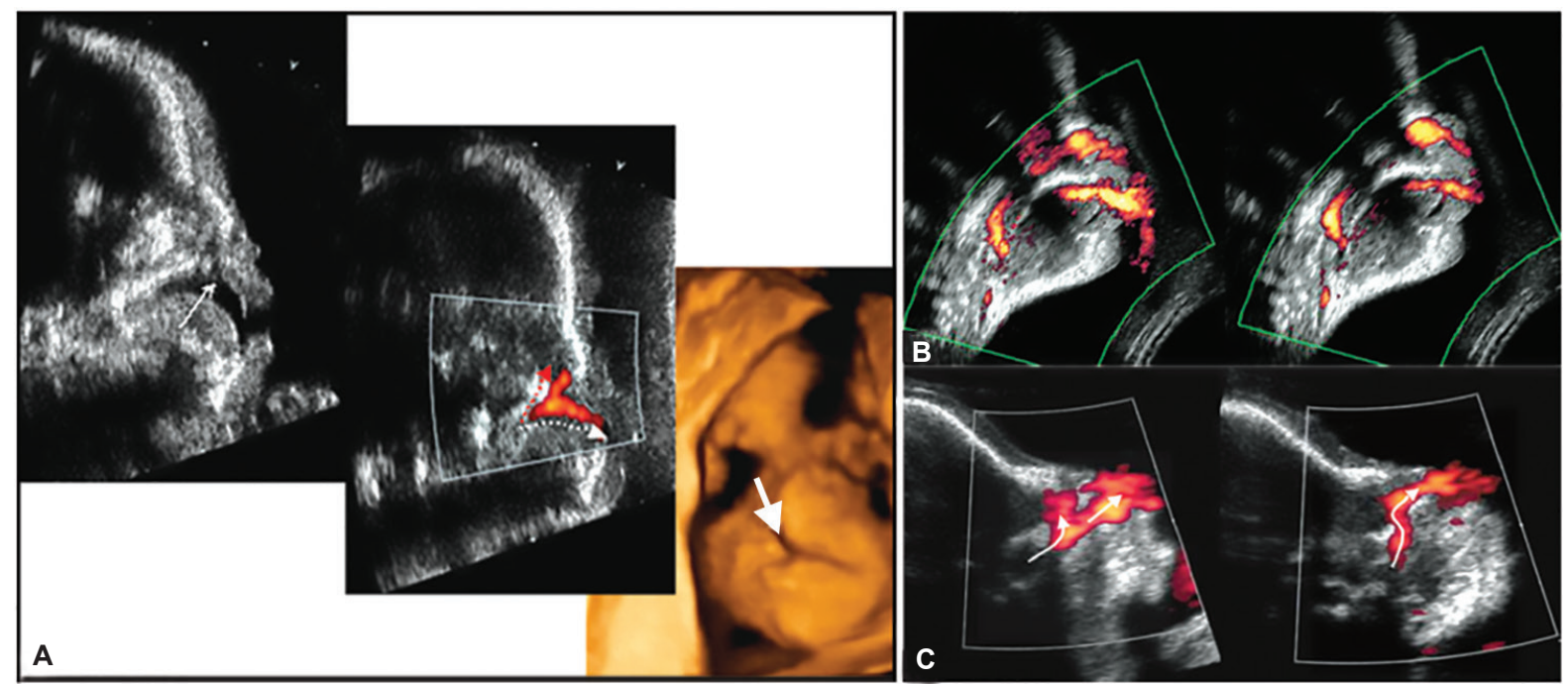

Figs 64A to C: $(A)$ The use of 2D, 3D and color Doppler offers a good possibility to diagnose the I-p cleft. It is suggestive of the passage of amniotic fluid directly from oral to nasal choana; (B) Sagittal scan power-color to demonstrate the normality of secondary palate during swallowing; (C) The amniotic fluid crosses through the palate

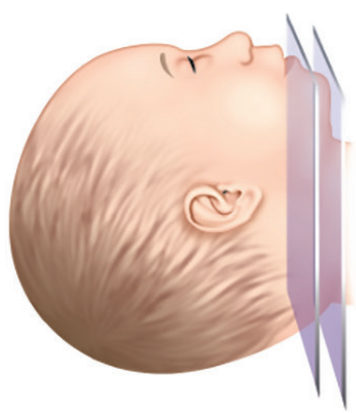

A
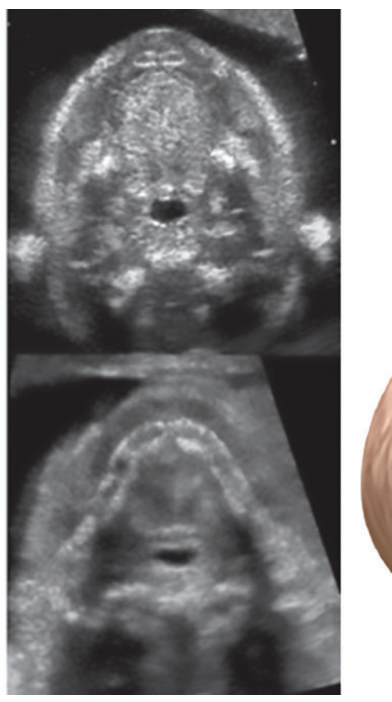

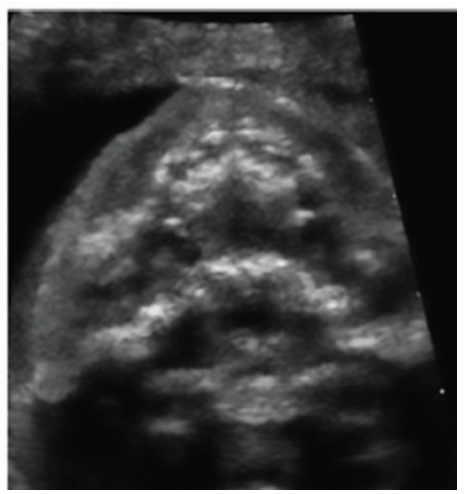

Figs 65A and B: Different planes of axial 2D scan for the jaw and the tongue. (A) The maxilla; (B) The proximal part of the maxilla and the eyes

has the ability to represent surfaces, as well as the VCI-cplane that makes it possible to dissect an orthogonal plane with ultrasound scanning by integrating multi-planar and rendering. ${ }^{95}$ A further step forward is the VCI-Omni view, which has the ability to model the source of 3D insonation by adapting to the structures of the fetal body that are normally represented by curved and straight lines (also the median line at the bottom is an artifact ultrasound). ${ }^{96}$ This technique allows direct and relatively simple scans which adapt to the adjustable thickness of the structures corpus studied (20 mm spine). There is no doubt that with a $3 \mathrm{D}$ to $4 \mathrm{D}$ instrument equipped with VCI-c-plane 

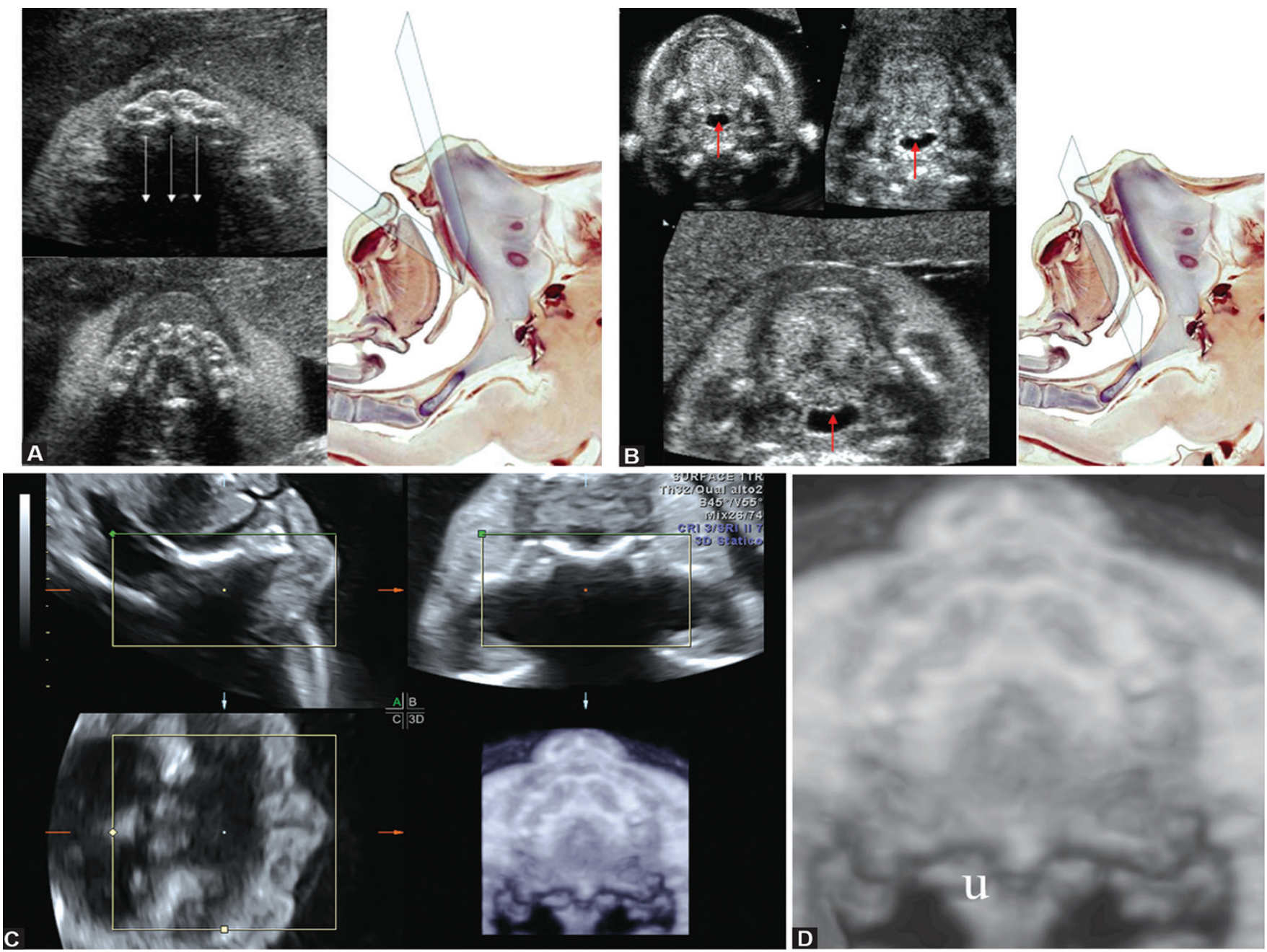

Figs 66A to D: (A) A correct axial 2D scan for the maxillary bone; $(B)$ The tongue and the uvula; (C) The secondary palate with the uvula by 3D flipped-face view multiplanar; (D) Surface rendering
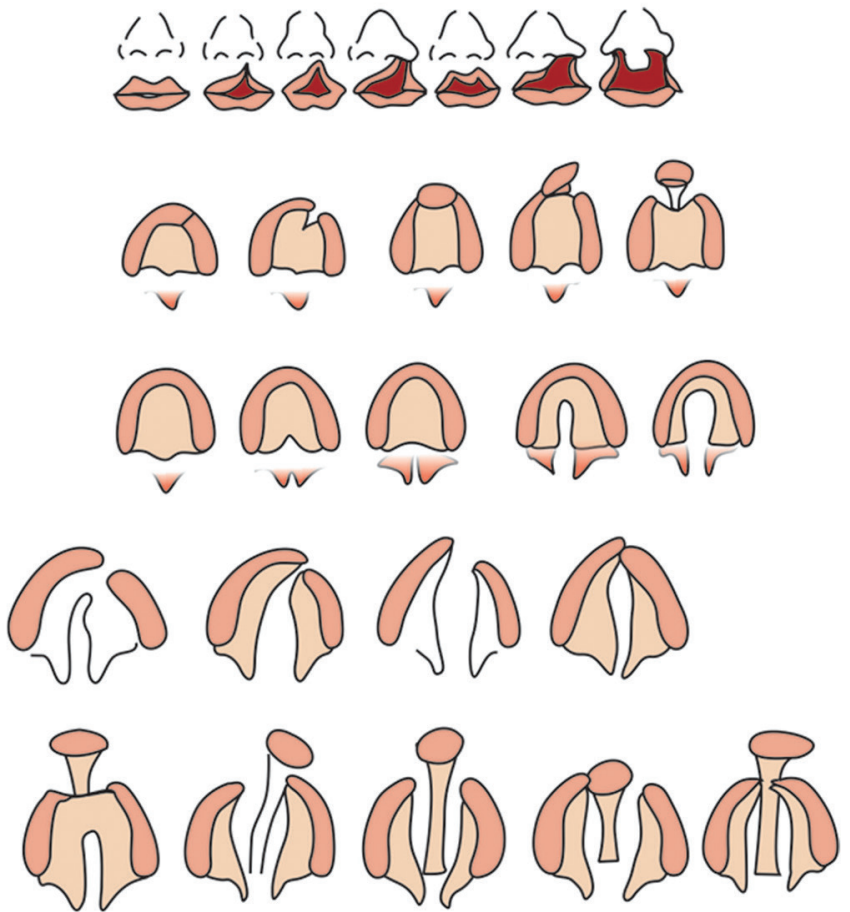

Fig. 67: Picture of Berkowitz (2006) shows the different possibilities of facial cleft. It is important to note that the palate-cleft starts from the uvula until the primary palate
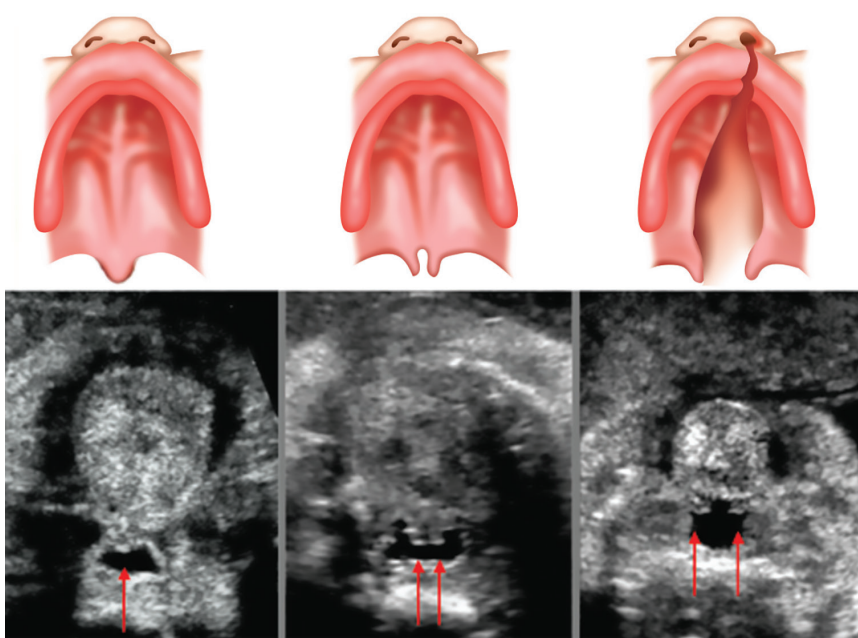

Fig. 68: Beautiful 2D pictures of uvula-cleft; the uvula-cleft is always associated with palate-cleft

adapted to insonation, can produce virtually overlaping results in terms of detecting anatomical structures in almost all three trimesters, also if with significantly reduced times using the omni-view in the second trimester. The VCI-Omni view, sharing the latest technological achievements, allows one to specify the anatomical study 


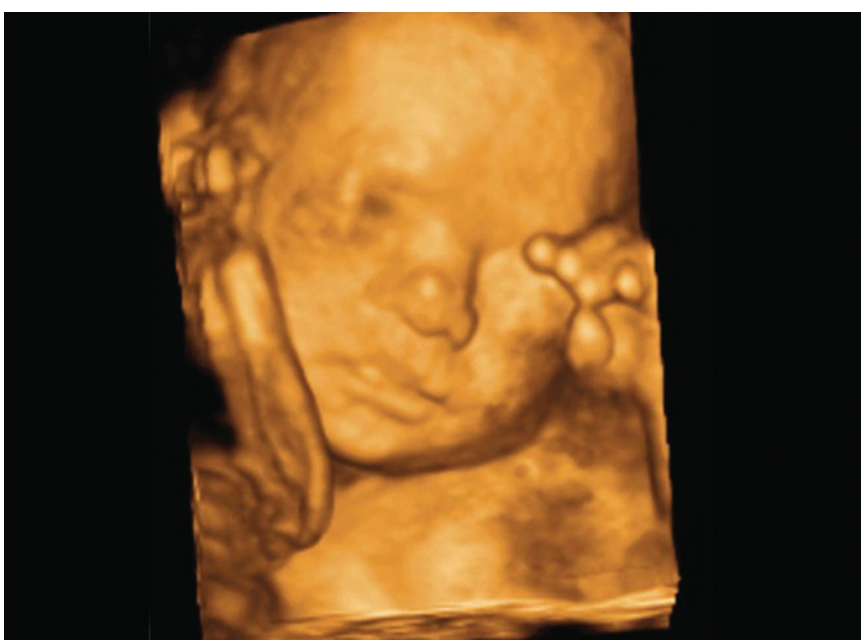

Fig. 69: The study of the nose and the lips is very easy in the second trimester of pregnancy by $3 \mathrm{D}$

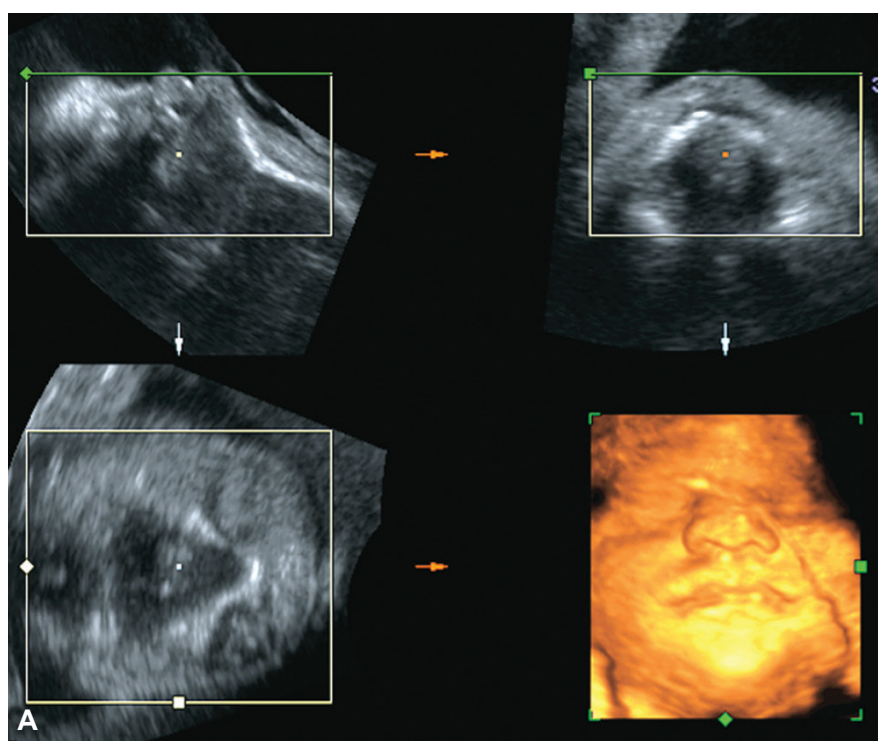

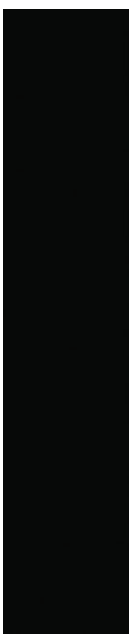

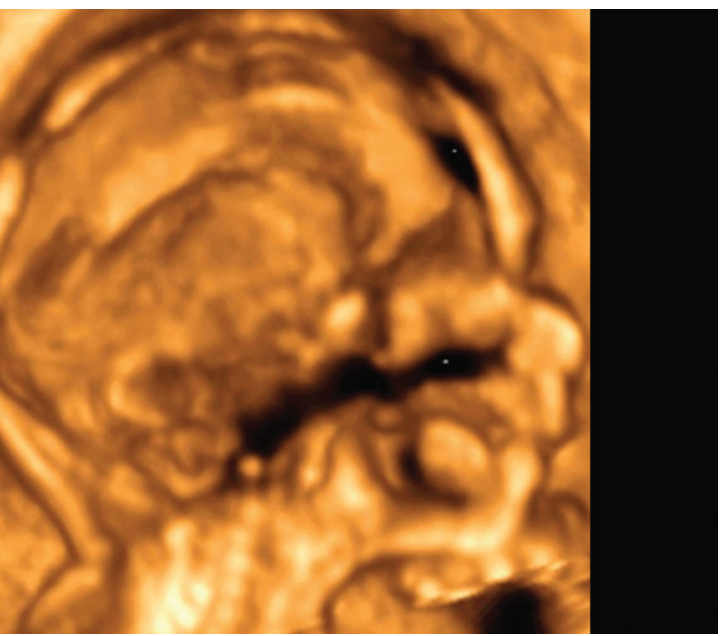

Fig. 70: The sagittal section of secondary palate shows the two portions of hard and soft palate with the uvula
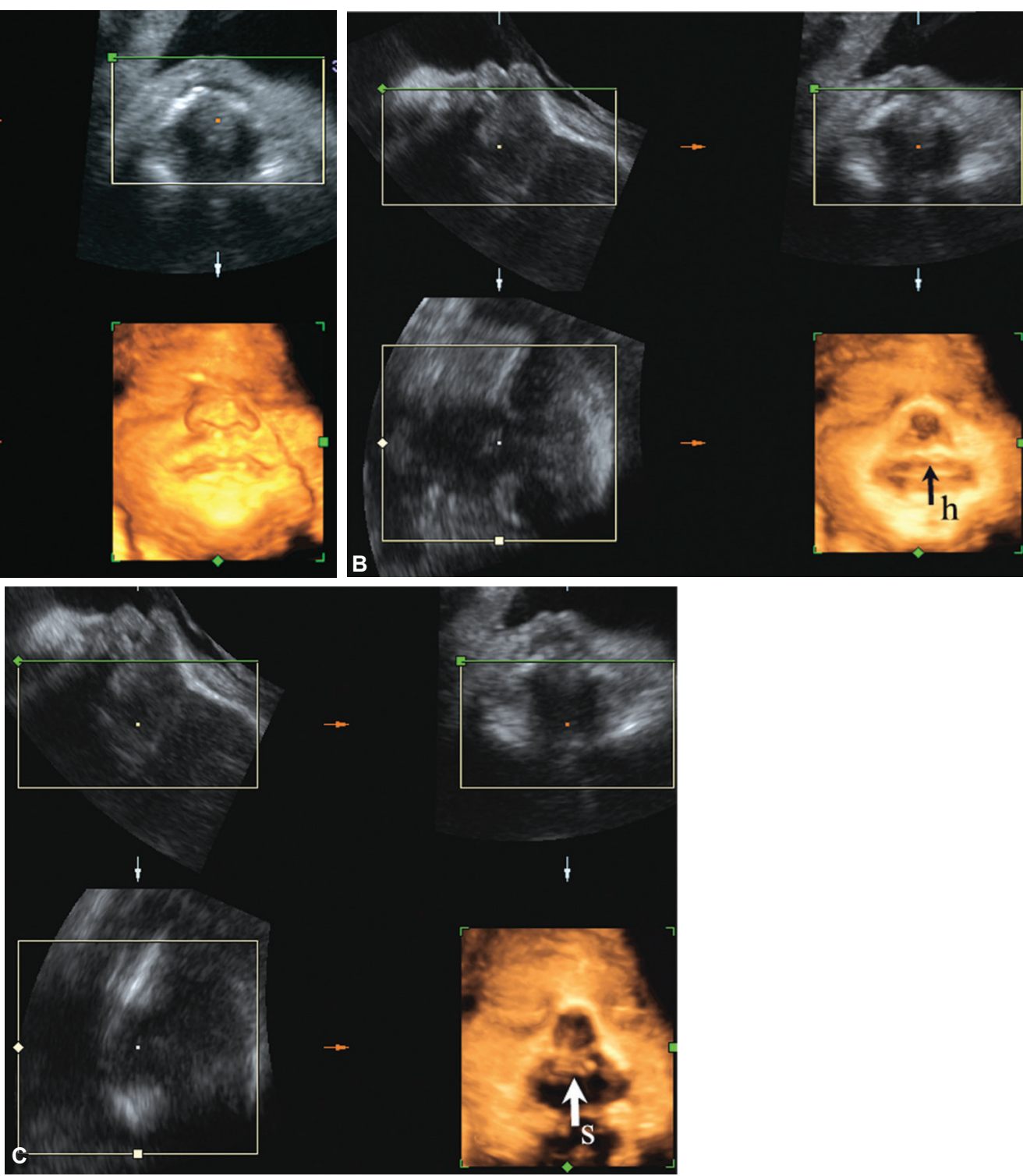

Figs 71 A to C: Secondary palate by coronal 3D plane multiplanar and surface rendering: scrolling backwards (Abbreviations: h, hard palate; s, soft palate) 

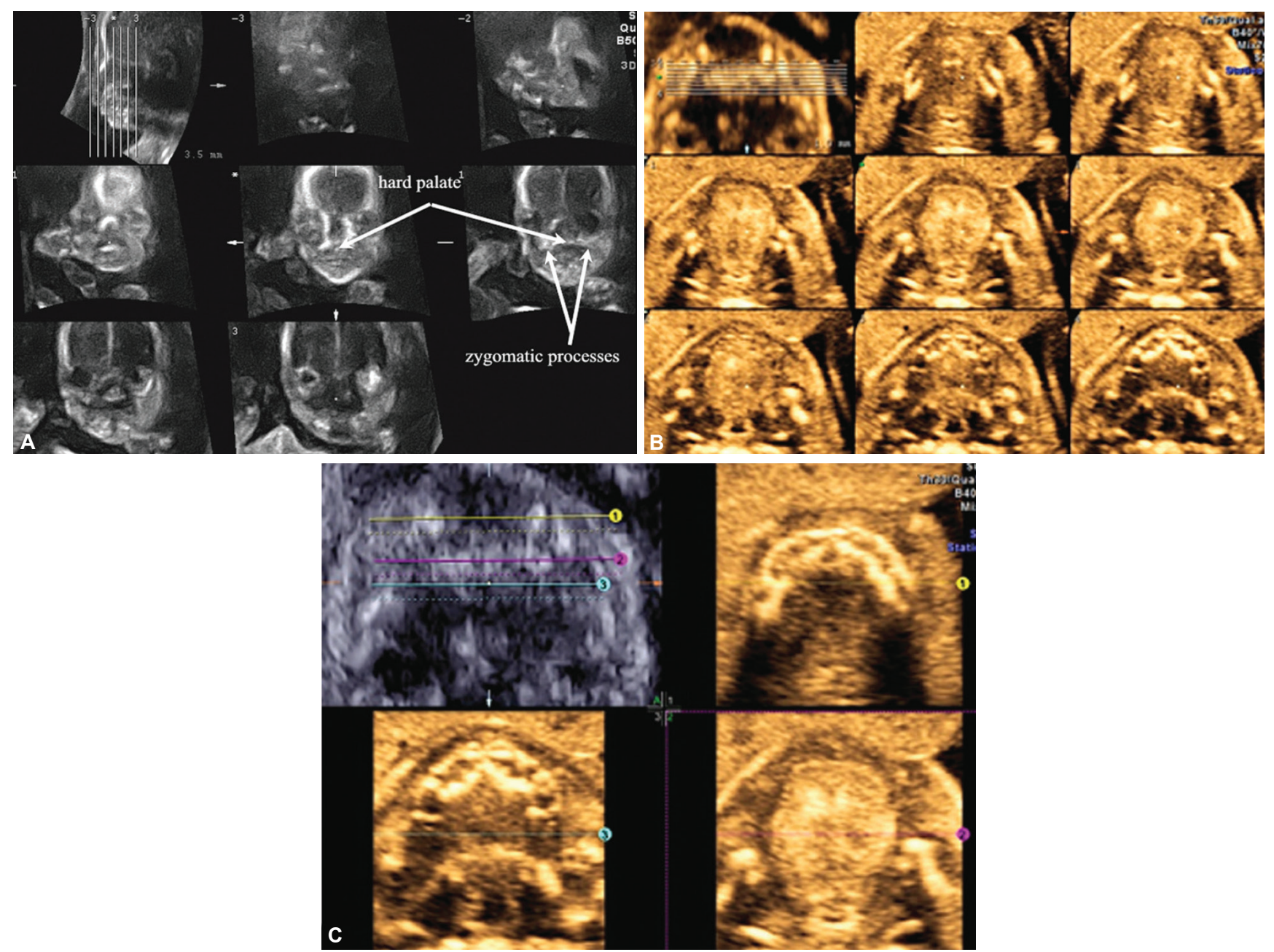

Figs 72A to C: (A) Tomographic ultrasound imaging (TUI) of the secondary palate in the coronal plane; (B) TUI of the secondary palate in the axial plane; (C) Omniview of the secondary palate in the axial plane
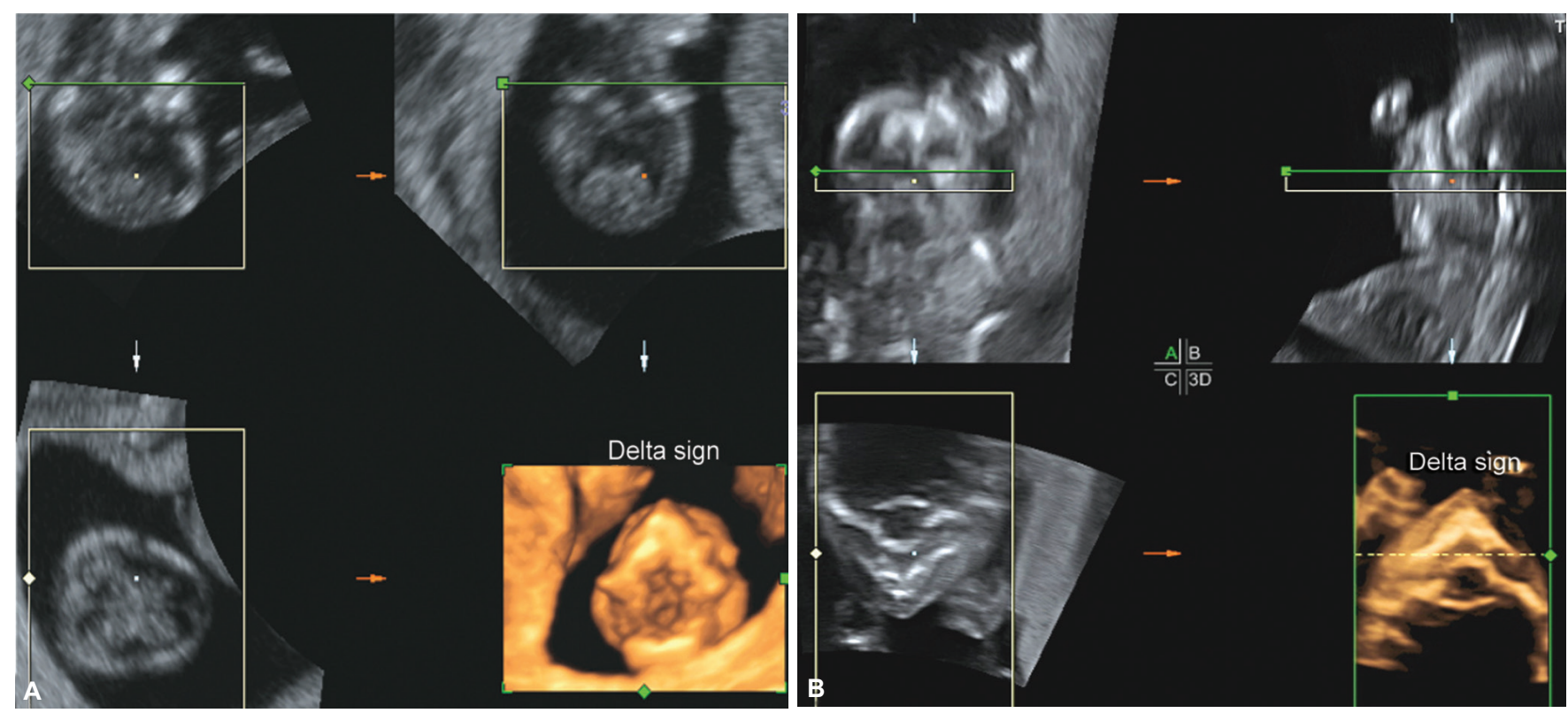

Figs 73A and B: Axial 3D plane multiplanar and surface rendering (flipped view) to see the maxilla (delta sign) and hard palate at 12 weeks by $(A)$ sagittal and $(B)$ coronal scan 


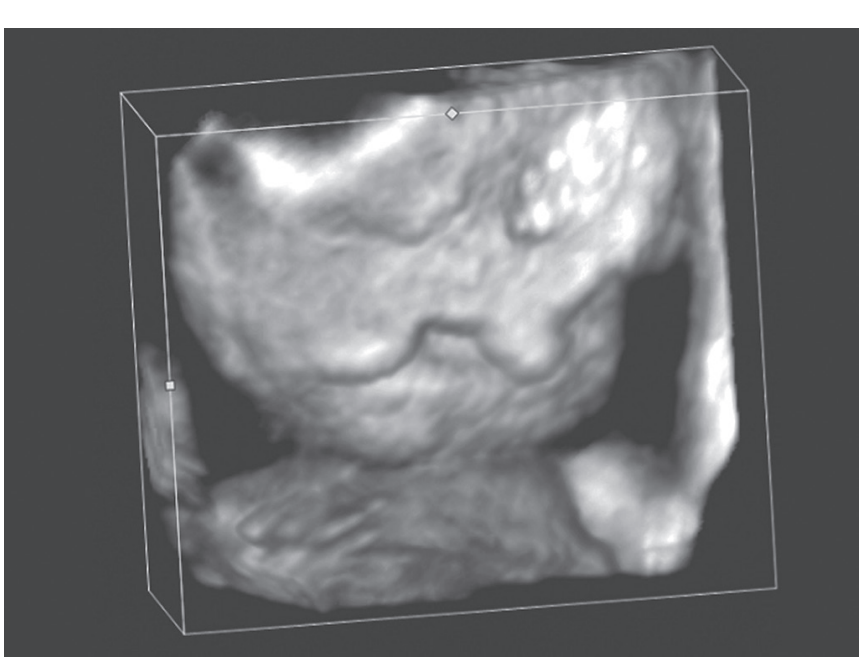

Fig. 74: A diagnosis at 14 weeks of cleft lip associated to holoprosencephaly by $3 \mathrm{D}$ vaginal probe $9-12 \mathrm{MHz}$ (E8 GE)

of the fetus by choosing the ideal section plane. Regarding the multiple planes, that being in 3D and 4D in the same volume, it can better yet follow the natural curves and angles of the structures, while rotating like a real scanner box that can focus on very small points, which would otherwise be very difficult to explore.

The detailed study is therefore quite superior since it can easily and quickly visualize the structures, that with $3 \mathrm{D}$ to $4 \mathrm{D}$ can be sometimes detectable only under particular conditions.

Study of the vertebral column and possible anomalies is much more convenient with 3-4D maximum mode which provides easy visualisation of all bones including the phalanges, as well as faster identification of any anomalies (platyspondyly, hemivertebrae, kyphosis, scoliosis such as absence (agenesis) or extra elements (Fig. 52 to 79). The ostechondrodysplasia have a prevalence of approximately 2.4 per 10,000 births and are rappresenatate in about $70 \%$ of four conditions: thanatophoric dysplasia, achrondroplasia, achondrogenesis, osteogeneisimperfecta Tip. II (Fig. 80).

The use of 3D doesn't offer a better condition to study the long bones, even if it is a good tool to store a volume and to explore by infinitive planes; but the study of dimension of the long bones, for instance the femur and the humerus for the suspicious of down syndrome is the same by $2 \mathrm{D}$ or $3 \mathrm{D}$. Instead the control of the extremity is a good field of exploration for three-dimensional scan. The club foot varus or valgus meet 415 syndromes in London medical databases; it is an important malformation because is often associated with other malformations or in about $2 \%$ with aneuploid malformation and in the last decade the detection rate of club foot achieve approximately $80 \%$ with about $15 \%$ of false positive rate. ${ }^{97}$ It is reasonable to think that the use of $3-4 \mathrm{D}$, by tools maximum mode and omni-view, will be possible improve the diagnosis of malformation of the extremity (feet and hands) and decrease the false positive rate, because three-dimensional scan is better in the study of anatomical detail (Figs 81 to 85 ).

\section{Genitals}

The genitals are a new field of study. It is relatively easy to diagnose sex in the second trimester by $2 \mathrm{D}$ and to determine descent of the testicles at 26-28 weeks (third trimester), however diagnosis of pathology of the external genitalia, such as hypospadia, is much easier with 3D (tulip sign $)^{98}$ (Fig. 86).

\section{Placenta and Umbilical Cord}

Detection of cord pathology (such as cysts), retrocervical position and number of twists is especially easy and reliable with power colour glass-body mode (Fig. 87). Also the study of position and vascularity of the placenta is easier by $3-4 D^{99,100}$ (Fig. 88).

The above is not intended as a scientific evaluation of the merits of 3-4D with respect to 2D, however almost all researchers report improved sensitivity and a better detection rate using 3-4D to measure volumes, determine orientation and definition of vessels and more precise definition of malformation, study coronal planes and the
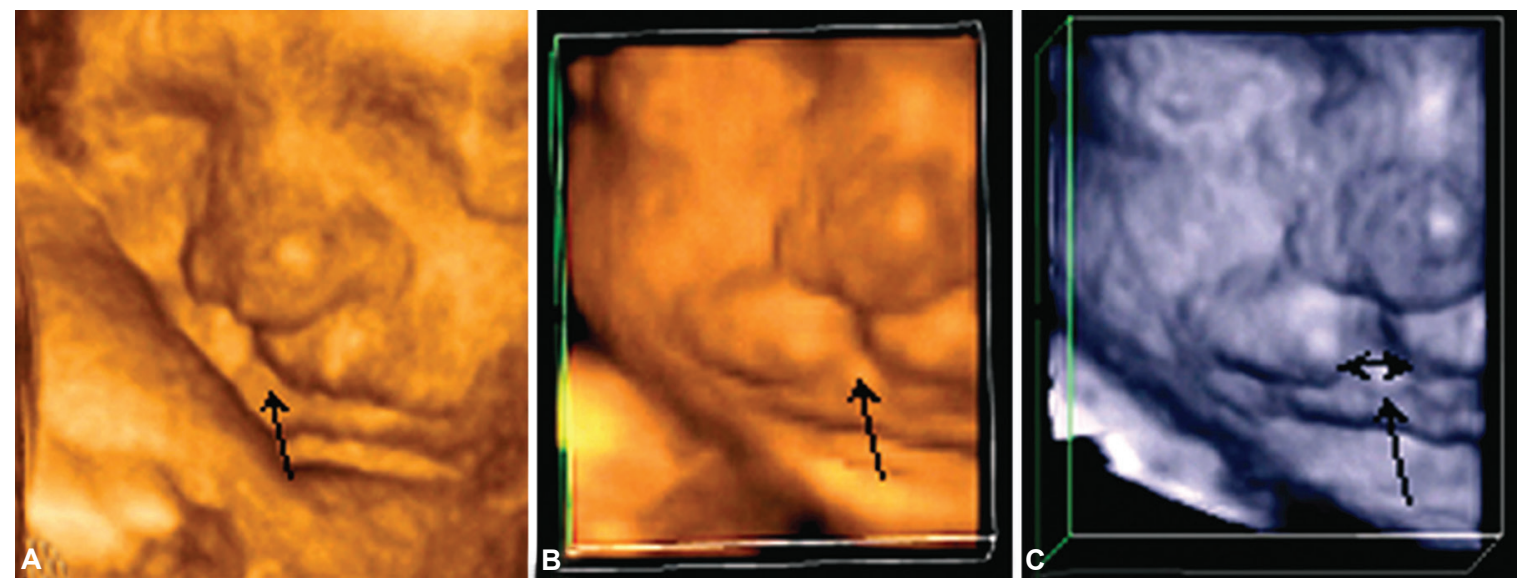

Figs 75A to C: Three images of cleft lip 


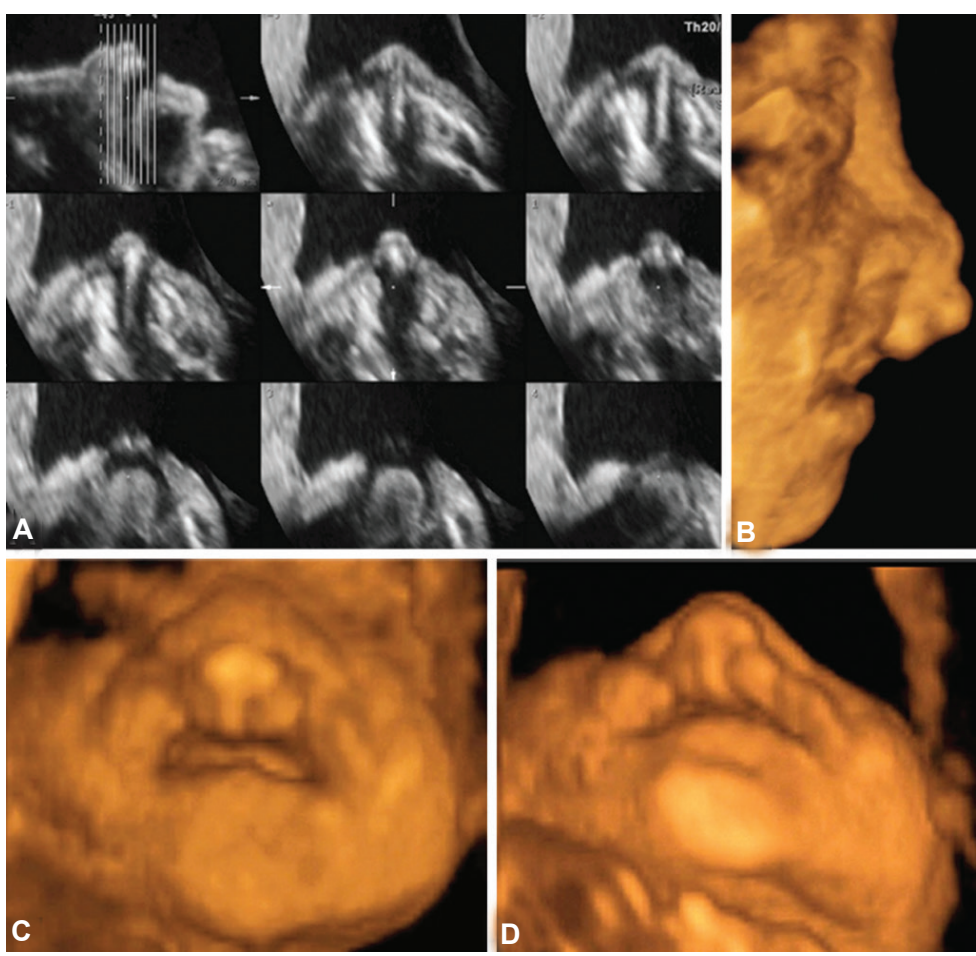

Figs 76A to D: Bilateral I-p cleft with TUI and rendering surface
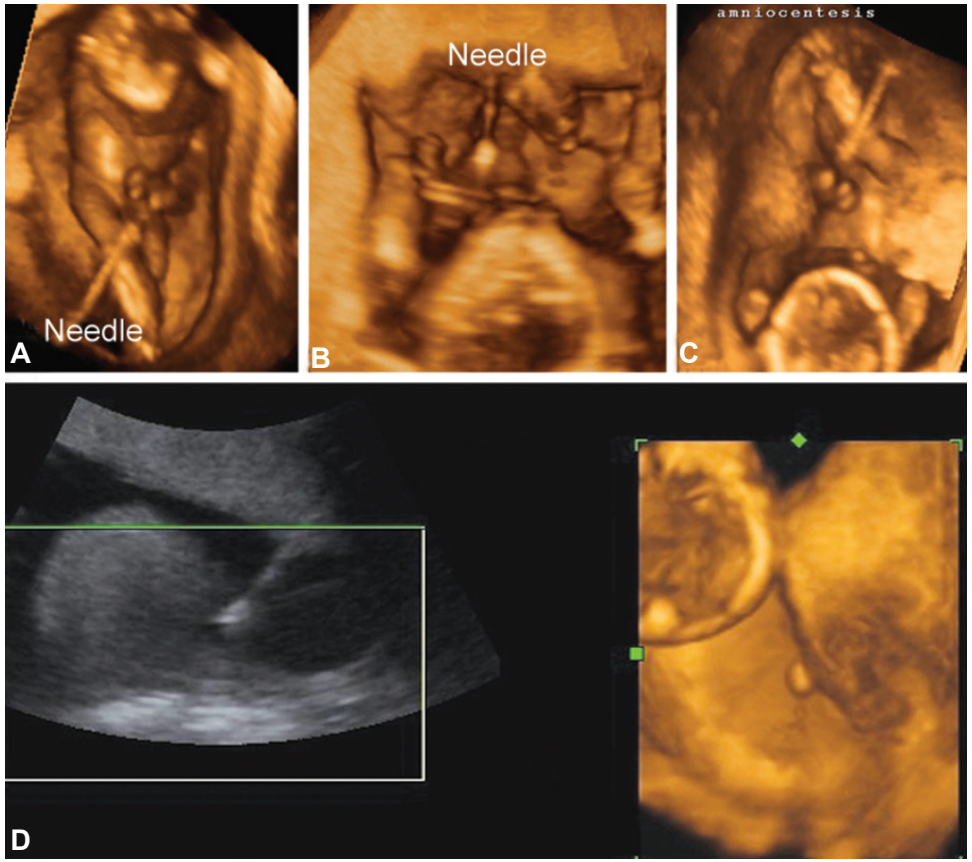

Figs 77A to D: (A to C) 3D images of amniocentesis; (D) Gives a good idea of the difference between 2D and 3D

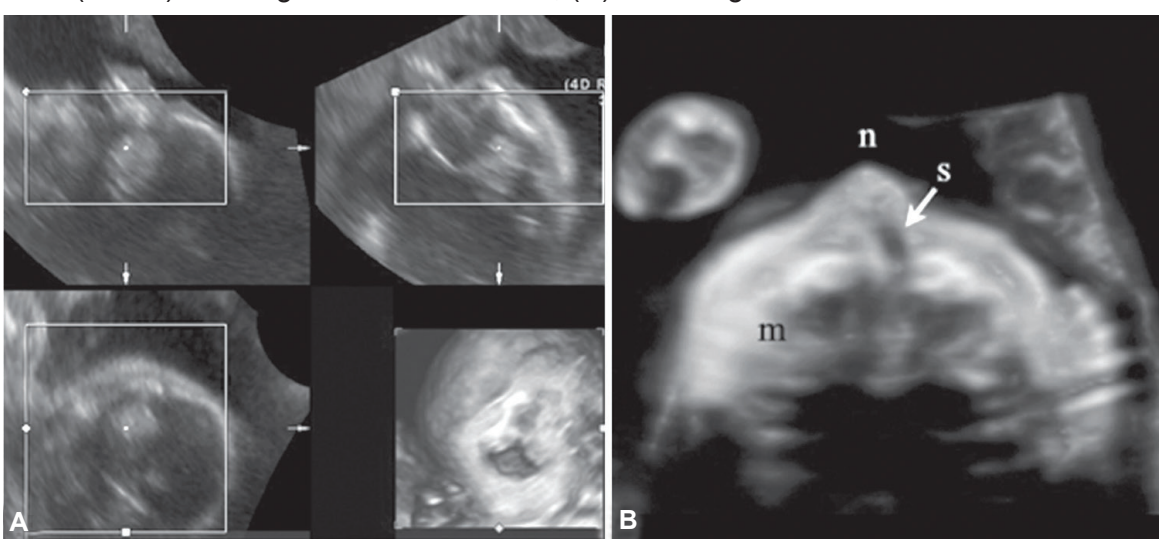

Figs 78A and B: The axial 3D plane by multiplanar and surface rendering "flipped view face" to see a cleft-lip cleft alveolar ridge 

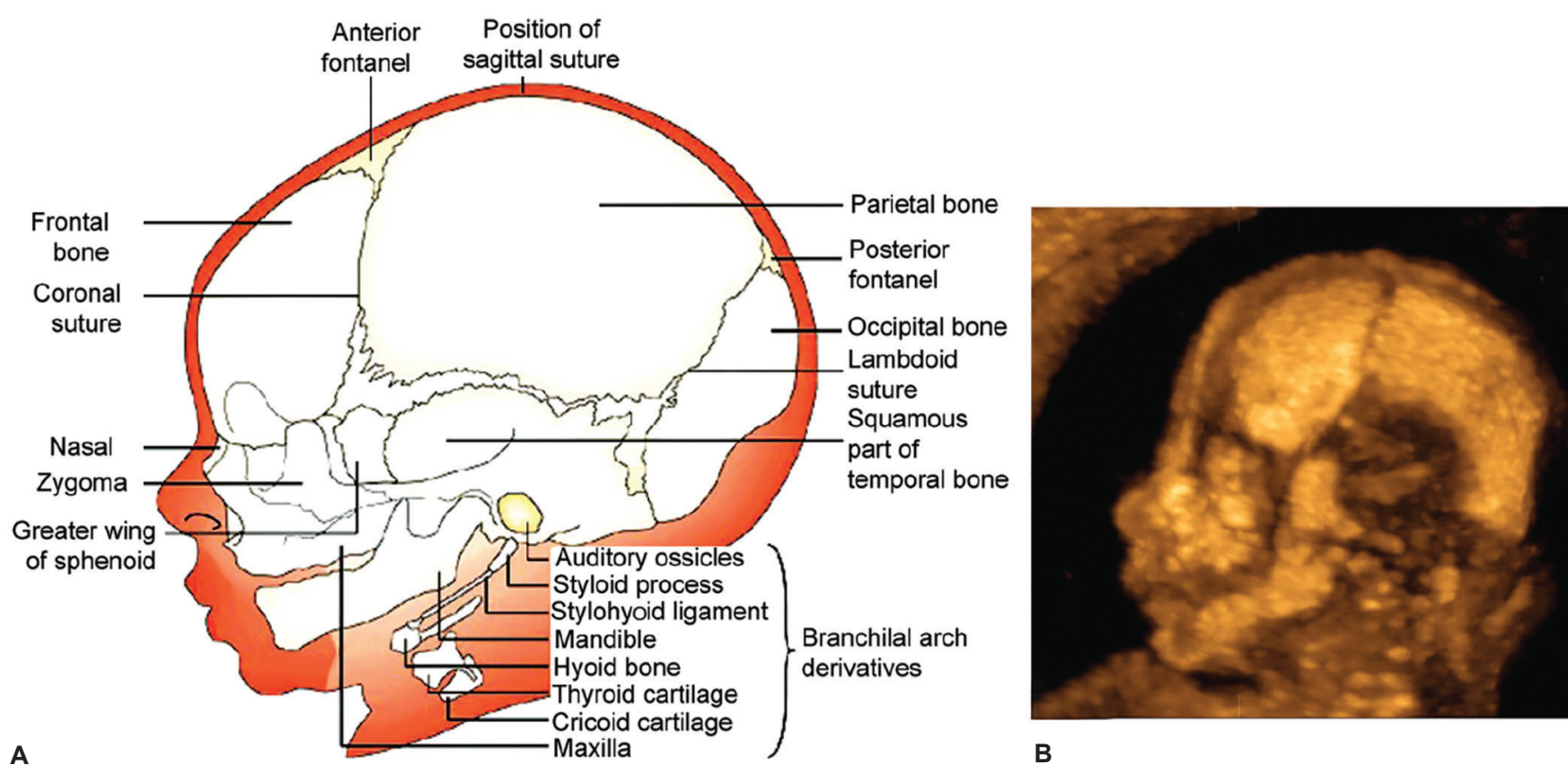

A

Maxilla

B
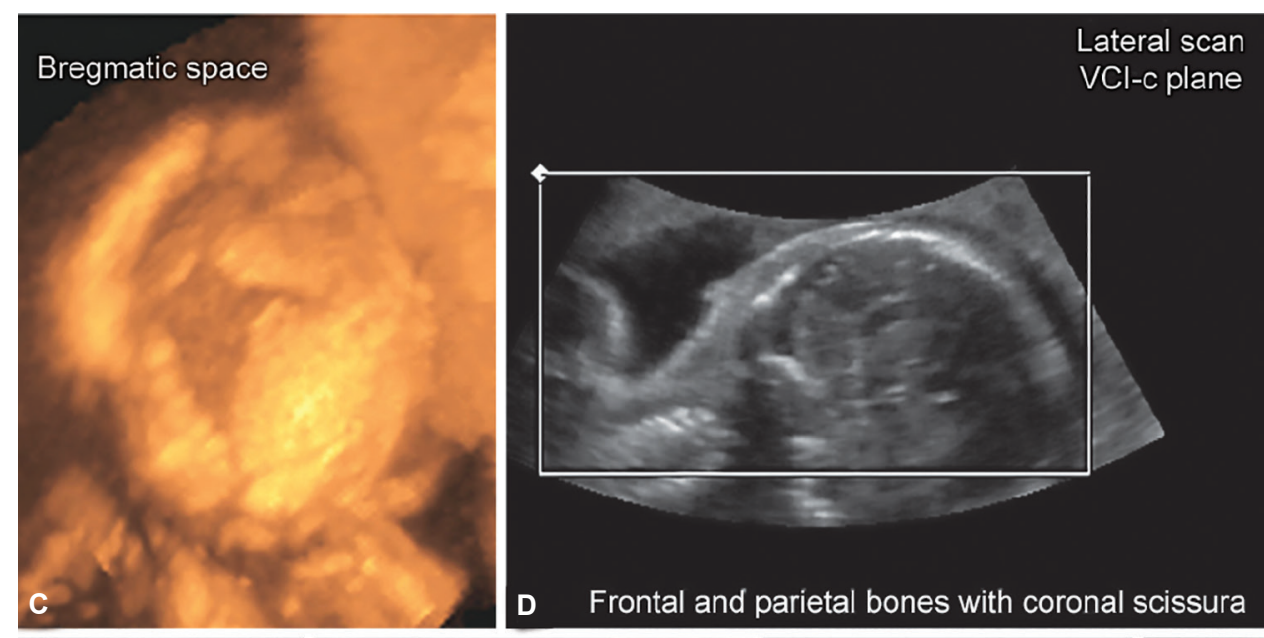

VI-c plane
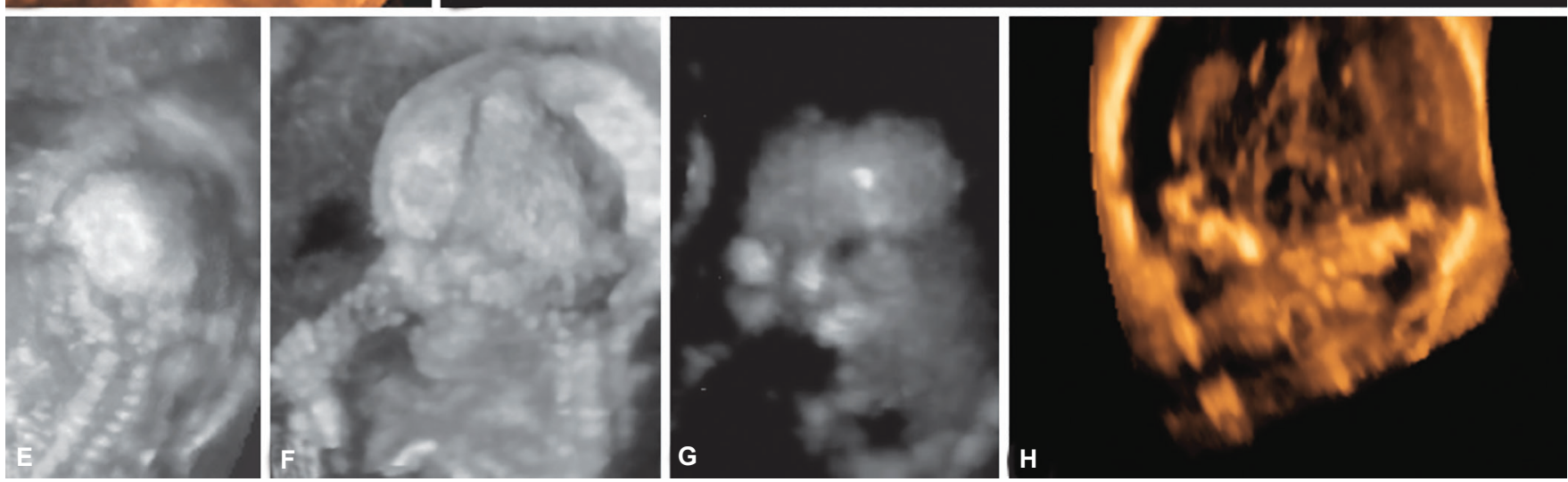

Figs 79A to $\mathrm{H}$ : (A to $\mathrm{C}$ ) The maximum mode imaging can detect so many features of the skull than it is possible to compare the image with a design; ( $D$ to $G$ ) Examination of the skull can detect many malformations: cranial bones and fissures are detectable by $3 D$, pathology of the bones like craniosynostosis at 20th week detected by maximum mode, or $(\mathrm{H})$ Sphenoid bone

foetal face, diagnose heart malformations (by STIC), visualise limbs and fingers and study embryo-fetal anatomy (especially neurosonology). All this does not establish a "need" for 3D for the structural study of the foetus, but certainly indicates that 3D instruments can improve ultrasound and make examination easier and more pleasant.

\section{Motor Activity and Facial Expression}

The structure of the foetal brain is in the process of development and in each phase of intrauterine life it has a maximum functional level. The first regions to mature are those necessary for life, such as sucking, swallowing and breathing. A first growth spurt is recognised between 


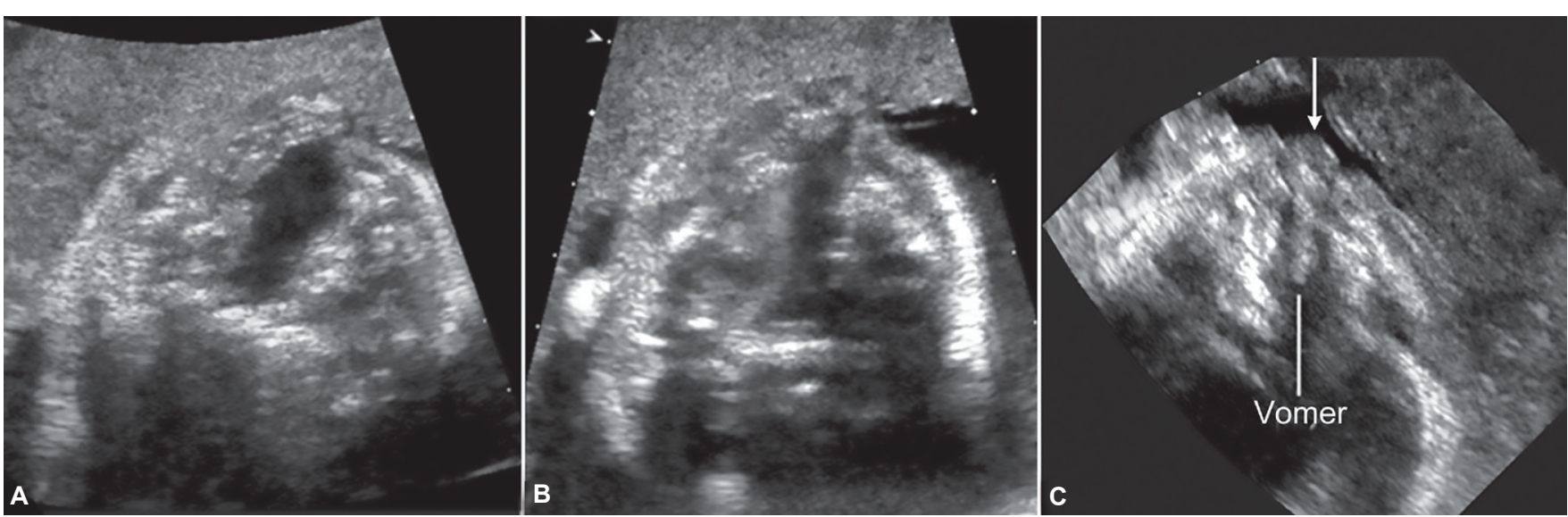

Figs $80 \mathrm{~A}$ to $\mathrm{C}$ : Monolateral cleft-lip and cleft-palate by axial 2D scan
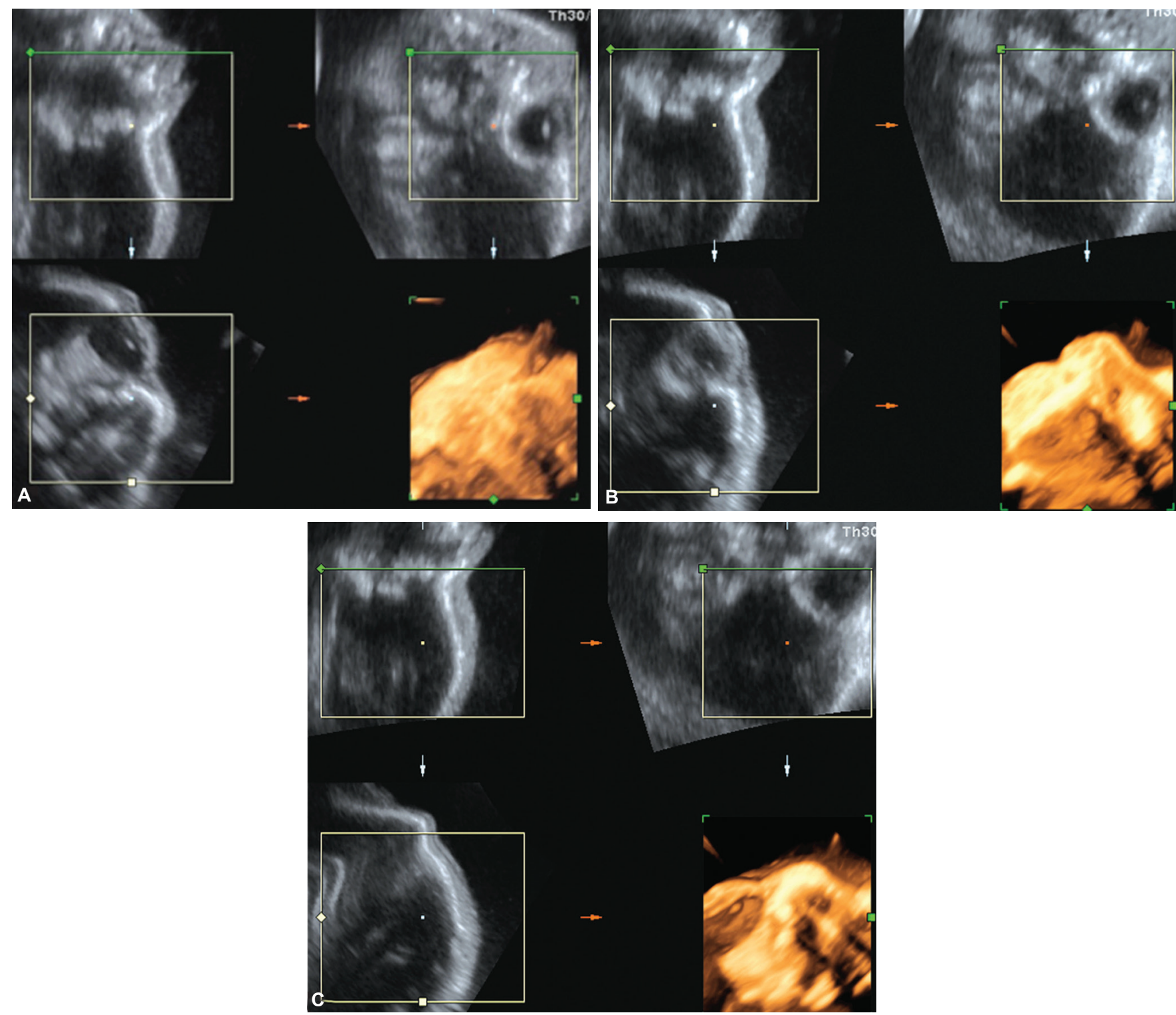

Figs 81A to C: Axial 3D plane, inverted image, "flipped view face", sagittal scan: (A) Cleft lip; (B) Cleft alveolar ridge; (C) Cleft palate

10 weeks of gestation and 18 months of neonatal life, with synapse formation and myelination.

Fetal movements can be divided into primary motor patterns and primary automatisms. The former are present in the first half of pregnancy and are genetically determined, whereas the latter depend on interaction of the foetus with its environment and occur from week 10 of gestation into the first years of estrauterina life. 

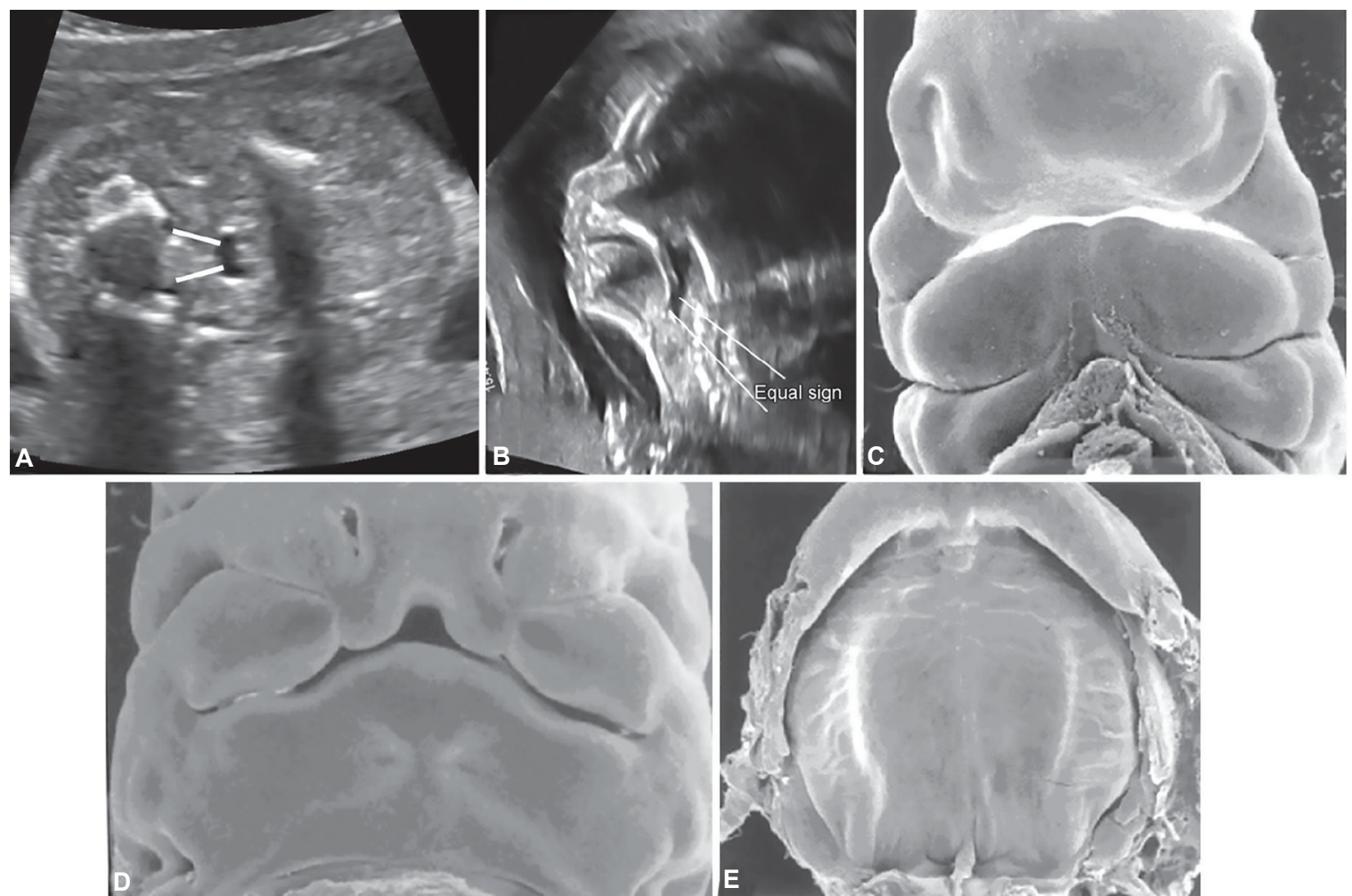

Figs $82 \mathrm{~A}$ to $\mathrm{E}:(\mathrm{A}$ and $\mathrm{B})$ Sagittal and axial scan offer a good possibility to see the "equal sign". The arrows indicate the two signs; (C) 6-weeks-old embryo: maxillary swellings fuse with medial nasal swellings, which merge with each other. The upper lip is still incompletely formed; (D) 53-day-old embryo: secondary palate: the fusion with primary palate has occurred; (E) 59-days-old embryo: complete fusion of the secondary palate has occurred. Courtesy: K Sulik, Chapel Hill, North Carolina
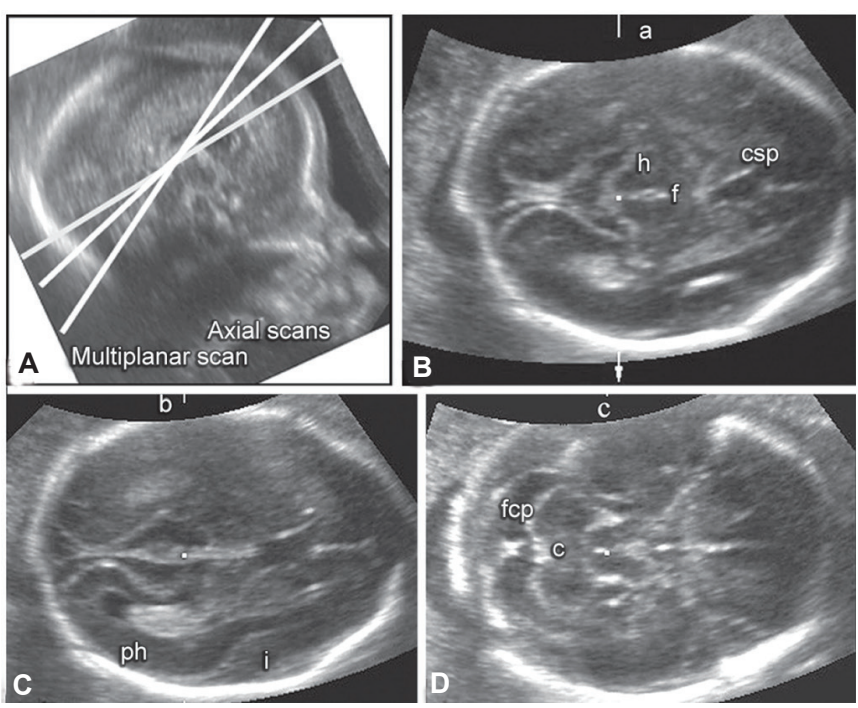

Figs 83A to D: Axial scans of fetal head. The three scans are the usual sections to measure BPD, to see the posterior horns and to measure the cerebellum

(Abbreviations: c, cerebellum; csp, cavum septi pellucidi; f, falx; pcf, posterior cranial fossa; ph, posterior horn; h, hypothalamus)

Active fetal movements increase with maturation of the nervous system, reaching a maximum at 32 weeks (after which they begin to decline); ${ }^{101}$ in the term foetus movements are similar to neonatal movements. Fetal circadian rhythm and foetal behavioural states such as quiet sleep, active sleep, quite waking state, active waking state and crying, are recognised. Alternation of these fetal behaviors in the 24-hour period is a sign of neurological maturation. Ultrasound can assess a foetal biophysical profile composed of foetal movements, muscle tone, respiratory movements, placental maturation and amount of amniotic fluid.

Doppler flow imaging plays a fundamental role in assessing foetal wellbeing, by evaluating alterations in blood flow in the placenta, foetus and mother. Robles de Medina et al. sustain that male and female fetuses do not show behavioural differences. ${ }^{102}$ Dynamic 3D scan (4D) can be useful together with 2D to study foetal behaviour in the second trimester of pregnancy. ${ }^{103}$

\section{Brain Function}

Though up to a few years ago the study of foetal brain function and hence motor activity, attitudes, posture and facial expression were of little interest, now, with $4 \mathrm{D}$ techniques that enable more accurate visualisation in real time of everything the foetus can do in utero, studies of foetal activity have become increasingly numerous. These studies are concerned with activity from the point of view of physiology as well as brain pathology. ${ }^{104}$ We now know much about foetal cognitive and emotional development from observation of premature babies who now survive from as early as 22 to 23 weeks of gestation. Clearly, estrauterine life involves stressors that force the premature baby along the path of brain development, often 


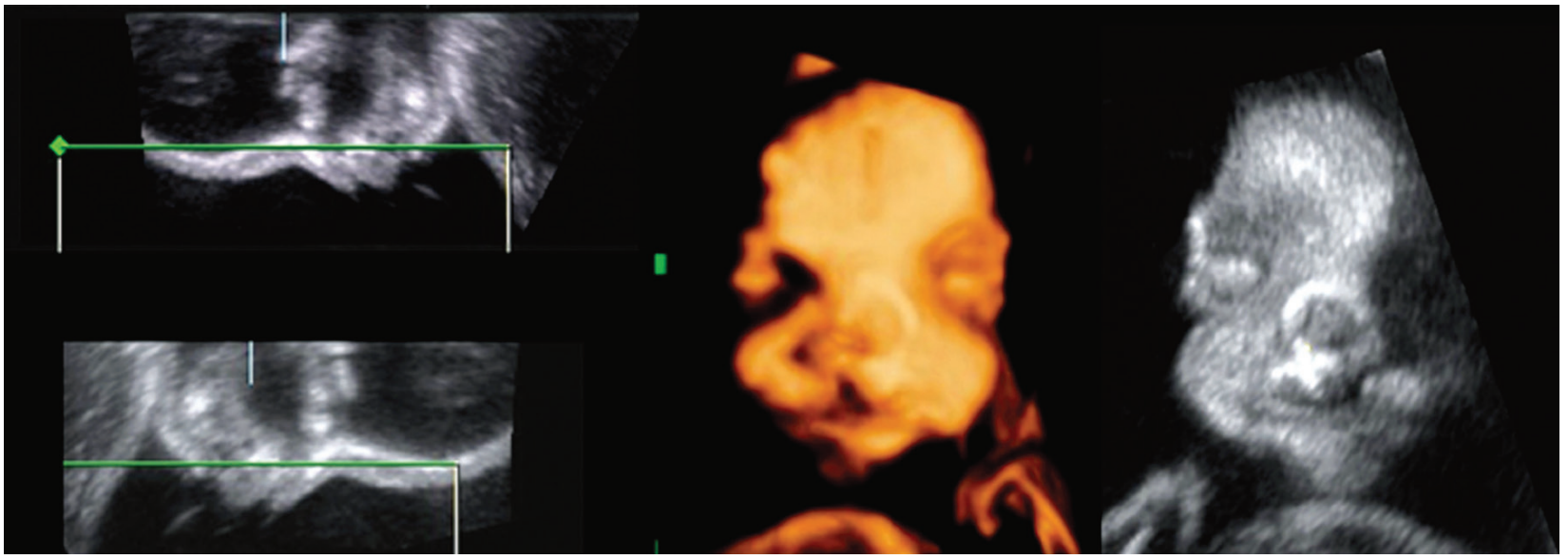

Fig. 84: Cleft-lip by "reverse face" mode and "front face" in the same case, of course change the position of the cleft
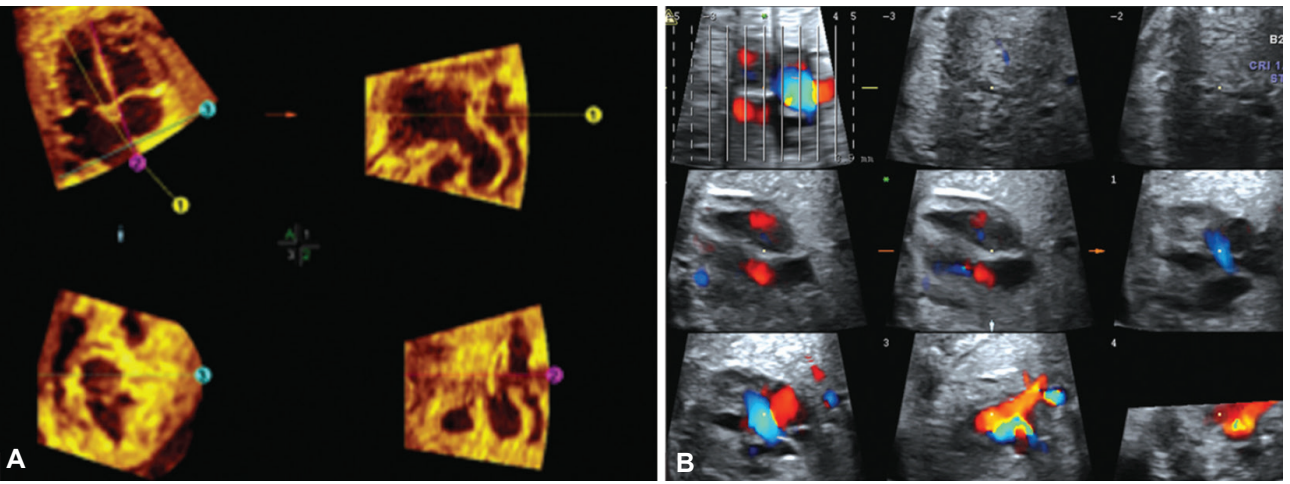

Figs 85A and B: The omniview and STIC are good tools to explore the heart
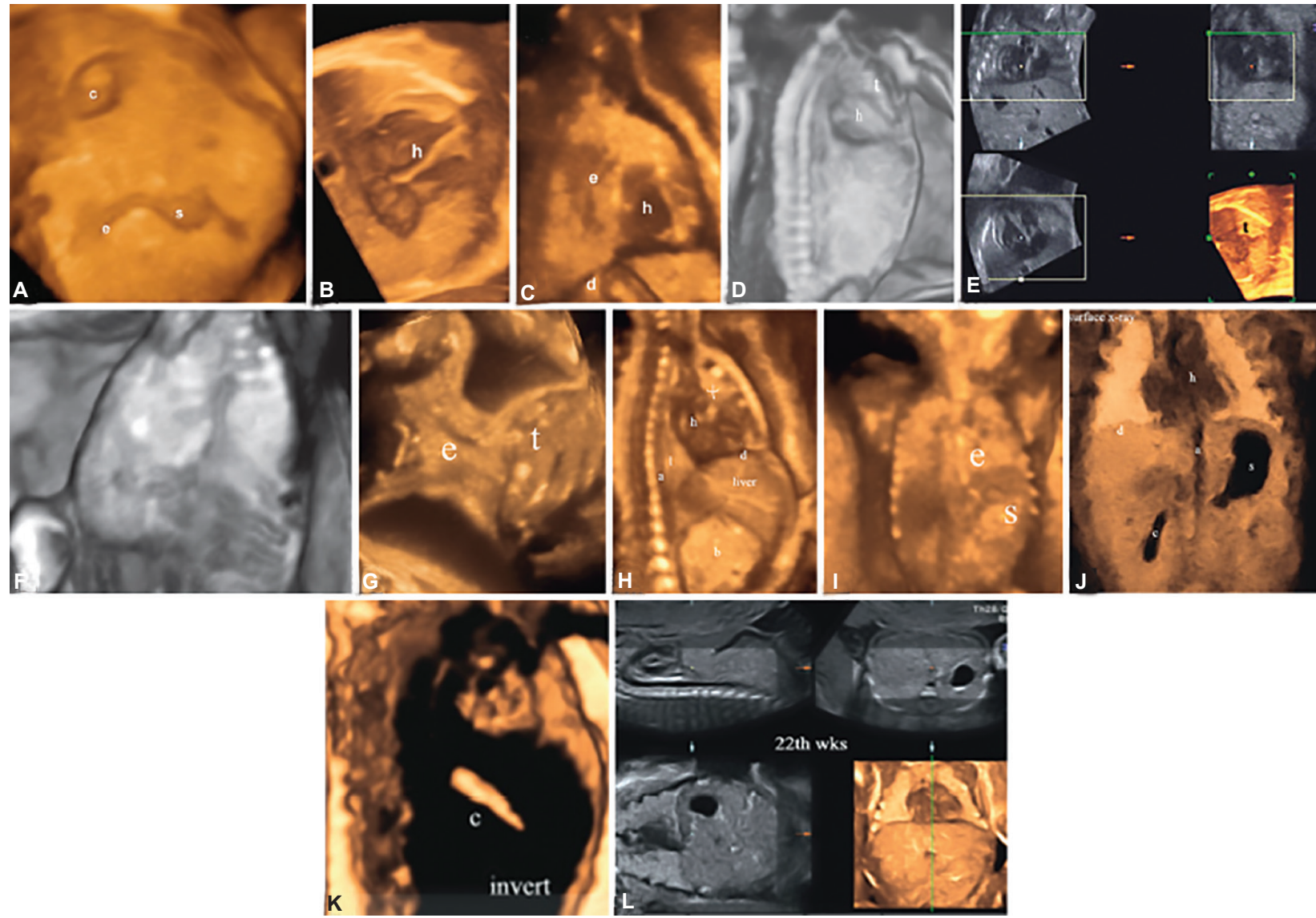

Figs $86 \mathrm{~A}$ to L: The chest and abdomen from the oral cavity to the esophagus are conveniently examined by 3-4D. The esophagus can be viewed from the oral cavity to the stomach by $3 \mathrm{D}$, aiding diagnosis of stenosis-agenesis. All organs (lung, diaphragm, heart, thymus and bowel) are realistically represented by $3 \mathrm{D}$

(Abbreviations: a, aorta; b: bowel; c, gallbladder; d, diaphragm; e, esophagus; $h$, heart; t, thymus) 


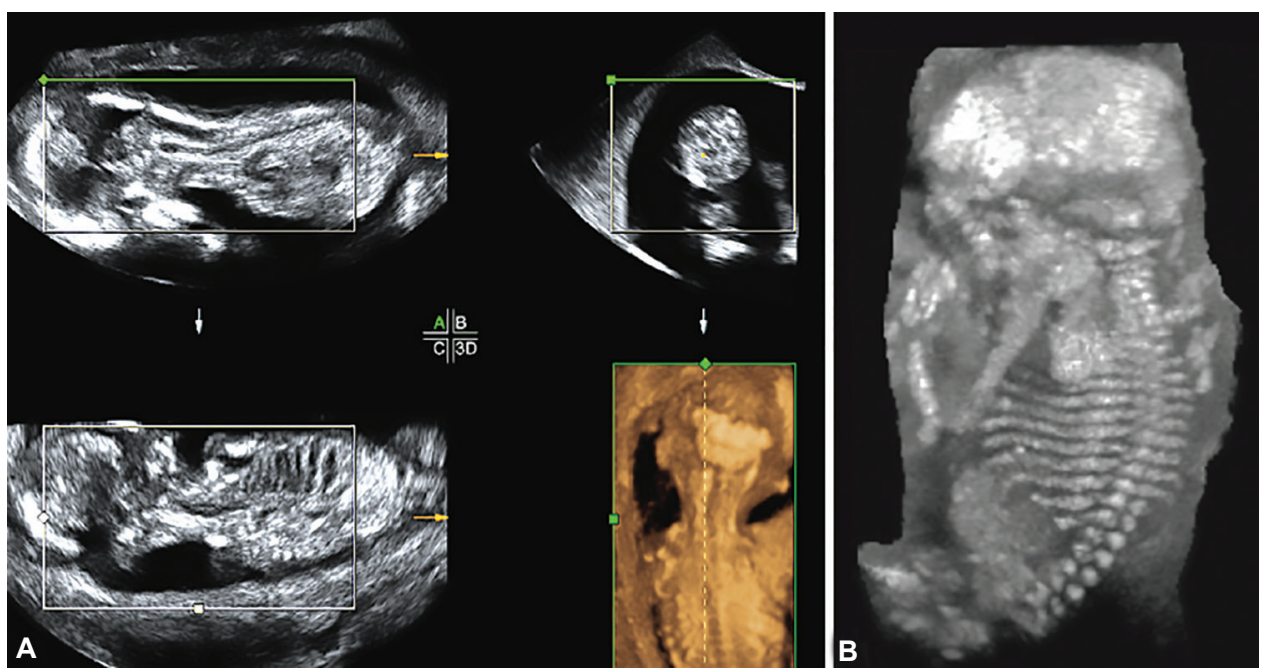

Figs $87 \mathrm{~A}$ and $\mathrm{B}$ : It is possible in the first trimester to study the bones of the skull. (A) Occipital bone at 12 weeks or the total skeleton with the spina; (B) The skeleton at 12 th weeks by maximum mode

with irremediable delays due to anoxia. Observation of premature babies therefore cannot be taken as validation of the physiological evolutionary milestones of the foetal brain. Renewed interest in this field has been spearheaded by Kurjak, who foresees major clinical benefits for $4 \mathrm{D}$ study of foetal behaviour. We personally have studied the moment when laterality, the predominance of one hemisphere over the other, is established. Laterality has been the same all over the world for thousands of years.
No scientific explanation of the predominance of the left over the right hemisphere has yet been found, if not an ancestral genetic mutation. Today there have been many papers, especially from Scandinavia, associating predominance of the right hemisphere, and hence predominant use of the left side of the body, with a significant increase in mental disorders. $4 \mathrm{D}$ scan enabled us to determine laterality in about $90 \%$ of fetuses between weeks 10 and 11 .
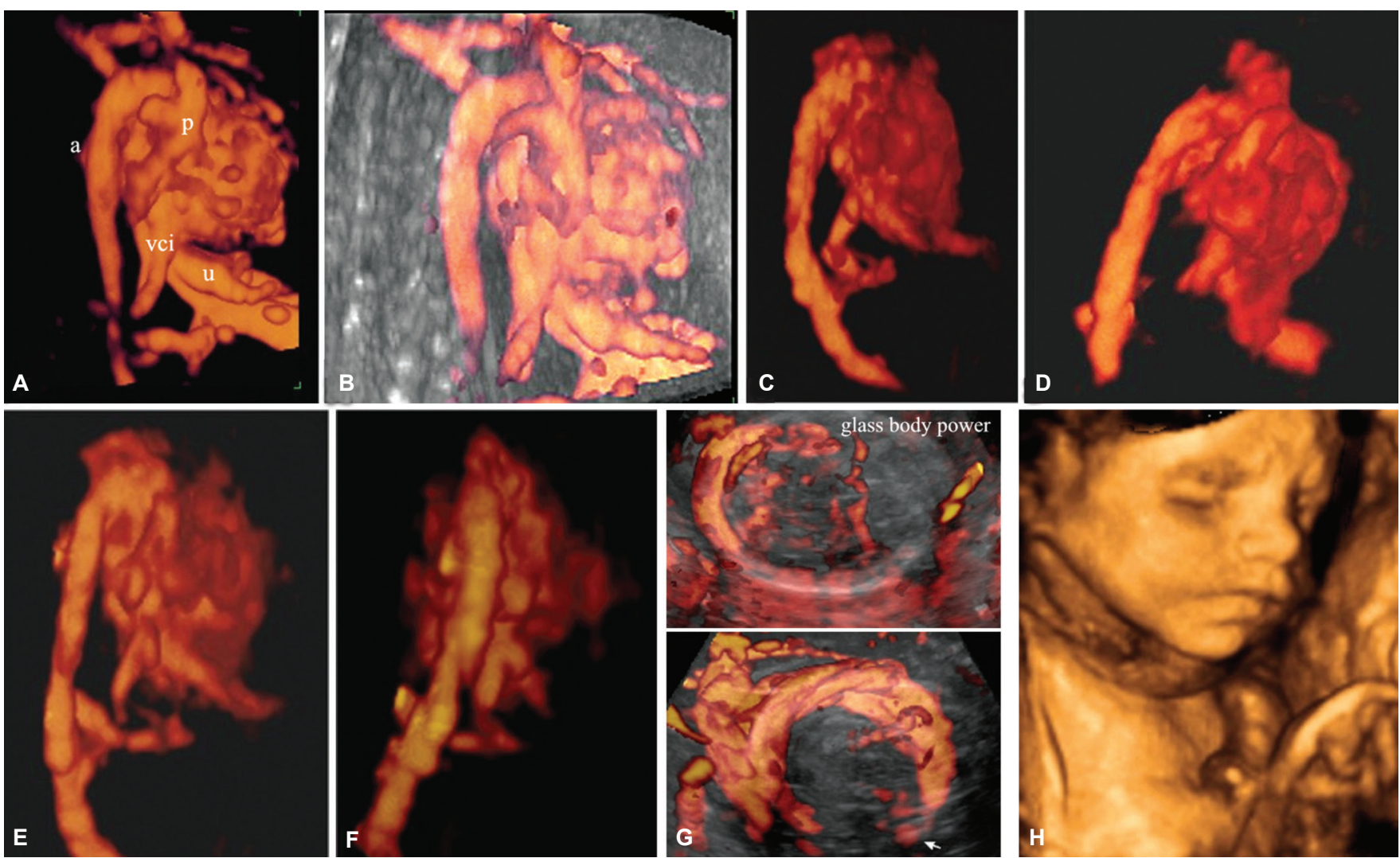

Figs $88 \mathrm{~A}$ to $\mathrm{H}$ : The glass body tool enhance the fetal circulation and thoracic conformation (Abbreviations: a, aorta; $p$, pulmonary artery; vci, inferior vena cava; u, umbilical artery 


\section{Behavior, Senses and Response to Stimulation in the Second and Third Trimesters}

As mentioned, much is now known about foetal behaviour at various gestational ages from the study of premature babies. However, the possibility of observing the foetus in its natural habitat in real time by 3-4 D has led much research that may help us to assess foetal wellbeing and specifically, the degree of neurological development in physiological situations and in the presence of CNS pathology. It is fairly easy to detect a foetus who yawns, puts out its tongue, touches itself and its surroundings, developing its sense of touch, pulls faces after ingesting amniotic fluid (taste), opens its eyes (attempts at seeing?), responds to sounds (hearing), responds to manual stimulation (many sustain that parental stimulation of the foetus by stroking or patting the maternal abdomen leads to faster and more intense development of neuronal function), starts (from weeks 11 and 12 the foetus can be observed reacting whenever its fingers and toes touch the wall of the uterus), hiccups, smiles, grimaces, frowns and expresses pain or serenity. The foetus therefore sends us many messages through its behaviour. Though much progress has been made, no scientifically demonstrated clinical applications of behaviour have yet been developed. It is certainly fascinating for parents and specialists to observe a foetus by $4 \mathrm{D}$, and it reinforces the hedonistic aspect of ultrasound examination in pregnancy.

\section{REFERENCES}

1. Dyson RL, Pretorius DH, Budorick NE, Johnson DD, Sklansky MS, Cantrell CJ,Lai S, Nelson TR. Three-dimensional ultrasound in the evaluation of fetal anomalies. Ultrasound Obstet Gynecol 2000;16(4):321-328.

2. Merz E, Bahlmann F,Weber G, Macchiella D. Threedimensional ultrasonography in prenatal diagnosis. J Perinat Med 1995;23(3):213-222.

3. Merz E, Welter C. 2D and 3D Ultrasound in the evaluation of normal and abnormal fetal anatomy in the second and third trimesters in a level III center. Ultraschall Med 2005; 26(1):916.

4. Pilu GL, Ghi T. Preliminary experience with advanced volume contrast imaging (VCI) and OmniView obstetric and gynecologist ultrasound. GE Healthcare, 2010

5. Bonilla-Musoles F, Raga F, Osborne NG, Bonilla F Jr; Caballero O, Climent MT, Wallraf SH, Castello JC. Multimodality 3-dimensional volumetric ultrasound in obstetrics and gynecology with an emphasis in Hdlivetechnique. Ultrasound Q 2013;29(3):189-201.

6. Pooh RH, Kurjak A. Novel application of three-dimensional Hdlive imaging in prenatal diagnosis from the first trimester. J Perinat Med 2015;43(2):147-158.

7. Grigore M, Mares A. The role of Hdlive technology in improving the quality of obstetrical images. Med Ultraso 2013;15(3):209-214.

8. AboEllail MA1, Tanaka H1, Mori N1, Hanaoka U1, Hata T1.HDlive silhouette mode in antenatal diagnosis of jejunal atresia. Ultrasound Obstet Gynecol 2015 Sep 4.
9. Forsberg F, Berghella V, Merton DA, Rychlak K, Meiers J, Goldberg BB.Comparing image processing techniques for improved 3-dimensional ultrasound imaging. J Ultrasound Med 2010;29(4):615-619.

10. Michailidis GD, Papageorgiou P, Economides DL. Assessment of fetal anatomy in the first trimester using two- and threedimensional ultrasound. Br J Radiol 2002;75(891):215-219.

11. Xu HX, Zhang QP, Lu MD, Xiao XT. Comparison of twodimensional and three-dimensional sonography in evaluating fetal malformations. J Clin Ultrasound 2002;30(9):515-525.

12. Downey DB, Fenster A, Williams JC. Clinical utility of threedimensional US. Radiographics 2000;20(2):559-71.

13. Campbell S. Doppler and 3D ultrasound in infertility - do they alter the outcome for the patient? Ultrasound Ostet Gynecol Vol 22 suppl. 1;2003 pag. 24.

14. Rosignoli L, Periti E, Centini G. 3D Omniview sonography in the pre-assisted reproductive medicine programme. Ultrasound Obstet Gynecol 2010;36 (Suppl.1):52-167.

15. Timor-Tritsch IE, Fuchs KM, Monteagudo A, D'alton ME .Performing a fetal anatomy scan at the time of first-trimester screening. Obstet Gynecol 2009;113:402-407.

16. Hafner E, Metzenbauer M, Stümpflen I, Waldhör T, Philipp $\mathrm{K}$. First trimester placental and myometrial blood perfusion measured by 3D power Doppler in normal and unfavourable outcome pregnancies.Placenta 2010;31(9):756-763.

17. Souka AP, Pilalis A, Kavalakis Y, Kosmas Y, Antsaklis P, Antsaklis A. Assessment of fetal anatomy at the 11-14week ultrasound examination. Ultrasound Obstet Gynecol 2004;24:730-734.

18. Tonni G. Centini G, Taddei F. Can 3D uktrasound and doppler angiography arteries be included in second trimester ecocrdiograpphic examination? A prospective study on low-risk pregnancy population. Echocardiography 2009;26(7):815-822.

19. Centini G, Sollazzi S, Rosignoli L, Ciani V, Imperatore A, Petraglia F, Lituania M, Tonni G. Capacità diagnostica ecografica dei processi mal formativi nel primo trimestre di gravidanza: è proponibile l'ecografia morfologica dalla 11a alla 14a settimana? Il Ginecologo 2010;5(1-2):22-29.

20. Merz E. Spina bifida aperta--detection of a shallow defect of the spine by 3D sonography. Ultraschall Med. 2007;28(3):246-7.

21. Tonni G, Centini G. Three dimensional first-trimester diagnosis of alobarholoprosencephaly associated with onphalocele in a 46,xx fetus. Am J perinat 2006.

22. Chaoui R, Nicolaides KH. From nuchal translucency to intracranial translucency: towards the early detection of spina bifida. Ultrasound Obstet Gynecol 2010;35(2):133-138.

23. Chaoui R, Benoit B, Mitkowska-Wozniak H, Heling KS, Nicolaides KH. Ultrasound Obstet Gynecol 2009;34(3):249252.

24. Schramm T, Gloning KP, Minderer S, Tutschek B. 3D ultrasound in fetal spina bifida. Ultraschall Med 2008;29(5):289-290.

25. Bilardo CM, Muller MA, Zikulnig L, Schipper M, Hecher $\mathrm{K}$. Ductus venosus studies in fetuses at high risk for chromosomal or heart abnormalities: relationship with nuchal translucency measurement and fetal outcome. Ultrasound Obstet Gynecol 2001;17:288-294

26. Nicolaides KH. Nuchal translucency and other first-trimester sonographic markers of chromosomal abnormalities. Am J Obstet Gynecol. 2004 Jul;191(1):45.

27. Matias A, Gones C, Flack N, Montenegro N, Nicolaides KH. Screening for chromosomal abnormalities at 10-14 weeks: the 
role of ductus venosus blood flow. Ultrasound Obstet Gynecol 1998;12:380-384.

28. Borrell A, Martinez JM, Serès A, Borobio V, Cararach V, Fortuny A. Ductus venosus assessment at the time of nuchal translucency measurement in the detection of fetal aneuploidy. Prenat Diagn 2003;23:921-926.

29. Matias A, Montenegro N. Ductus venosus blood flow in chromosomally abnormal fetuses at 11 to 14 weeks of gestation. Seminars in Perinatology 2001;25:32-37.

30. Toyama JM, Brizot ML, Liao AW, Lopes LM, Nomura RMY, Saldanha FAT, Zugaib M. Ductus venosus blood flow assesment at 11 to 14 weeks of gestation and fetal outcome. Ultrasound Obstet Gynecol 2004;23:341-345.

31. Gezer C,Echin A, Gezer NS, Ertas IE, Avuc ME, Uyar I, Ciftci $\mathrm{S}$, Taner CE. Prenatal karyotype results of fetuses with nuchal edema, cystic hygroma, and non-immune hydrops. Clin Exp Obstet Gynecol 2015;42(5):586-589.

32. Chevernak FA, Isaacson G, Blakemore KJ, Breg WR, Hobbins JC, Berkowitz RL, Tortora M, Mayden K, Mahoney MJ. Fetal cystic hygroma: cause and natural history. N Engl J Med 1983;309:822.

33. Mathias B, Forrester BS, Ruth D. Merz MS. Descriptive epidemiology of cystic hygroma: Hawaii, 1986 to 1999 . Southern Medical Journal 2004;97:631-636

34. Bernstein HS, Filly RA, Goldberg JD, Golbus MS. Prognosis of fetuses with a cystic hygroma. PrenatDiagn. 1991;11:349-355.

35. Podobnik M, Singer Z, Podobnik-Sarkanji S, Bulic M. First trimestre diagnosis of cystic hygromata using transvaginal ultrasound and cytogenetic evaluation. J Perinat Med 1995;23:283-291.

36. Rosati P, Guariglia L. Transvaginal ultrasound detection of septated and non-septated cystic hygroma in early pregnancy. Fetal Diagn Ther 1997;12:132-135.

37. Farkas LG, Katic MJ, Forrest CR, Litsas L. Surface anatomy of the face in Down's syndrome: linear and angular measurements in the craniofacial regions.J Craniofac Surg 2001;12:373-379.

38. Stempfle N, Huten Y, Fredouille C, Brisse H, Nessman C. Skeletal abnormalities in fetuses with Down's syndrome: a radiographic post-mortem study. Pediatr Radiol 1999;29:682-688.

39. Rosignoli L. An early diagnosis of trisomy 18 by $2-3-4 \mathrm{D}$ at 10th week. Ultrasound obstet gynecol, London 3-7 September 2006.

40. Sonek J, Nicolaides KH. Prenatal ultrasonographic diagnosis of nasal bone abnormalities in three fetuses with Down syndrome. Am J Obstet Gynecol 2002;186:139-141.

41. Cicero S, Rembouskos G, Vandecruys H, Hogg M, Nicolaides $\mathrm{KH}$. Likelihood ratio for trisomy 21 in fetuses with absent nasal bone at the 11-14 weeks scan. Ultrasound Obstet Gynecol 2004;23:218-223.

42. Kelekci S, Yazicioglu HF, Oguz S, Inan I, Yilmaz B, Sommez $\mathrm{S}$. Nasal bone measurement during the first trimester: Is it useful? Gynecol Obstet Invest 2004;58:91-95.

43. Peralta CF, Falcon O, Wesrzyn P, Faro C, Nicolaides KH. Assessment of the gap between the fetal nasal bone at 11 to $13+6$ weeks of gestation by three-dimensional ultrasound. Ultrasound Obstet Gynecol 2005 May; 25(5):464-467).

44. Cicero S, Binda R, Rembouskos G, Spencer K, Nicolaides. KHIntegrated ultrasound and biochemical screening for trisomy 21 at 11 to 14 weeks. PrenatDiagn 2003;23:306310.
45. Benoit B, Chaoui R. Three dimensional ultrasound with maximal mode rendering: a novel technique for the diagnosis of bilateral or unilateral absence or hypoplasia of nasal bones in second-trimester screening for Down syndrome. Ultrasound Obstet Gynecol 2005;25(1):19-24.

46. Cicero S, Curcio P, Rembouskos G, Sonek J, Nikolaides $\mathrm{KH}$. Maxillary length at 11-14 weeks gestation in fetuses with trisomy 21. Ultrasound Obstet Gynecol 2004;24(1):1922.

47. Rembouskos G, Cicero S, Sacchini C, Nikolaides KH. Single umbilical artery at 11-14 weeks of gestation: relation to chromosomal defects. Ultrasound Obstet Gynecol 2003; 22(6):567-570.

48. Sollazzi S, Centini G, Ciani V, Imperatore A, Servillo R, Rosignoli L. First trimester ultrasonography in detection of fetal anomalies. Ultrasound Obstet Gynecol 36(1):119.

49. Guidelines SIEOG 2010. ISBN 88-6135-124-7;978-88-6135-124-0

50. Eurocat Group. Prenatal diagnosis of severe structural congenital malformations in Europe. Ultrasound Obstet Gynecol 2005;25:6-11.

51. Rosignoli L, Tonni G, CentiniG.Cranial development in the first trimester: the use of 3D in the study of complex structures. Imaging Med 2010,2(3),251-257.

52. Rotten D, Levaillant JM. Two- and three-dimensional sonographic assessment of the fetal face. 1. A systematic analysis of the normal face. Ultrasound Obstet Gynecol 2004; 23(3):224-231.

53. Rotten D, Levaillant JM. Two- and three-dimensional sonographic assessment of the fetal face. 2. Analysis of cleft lip, alveolus and palate. Ultrasound Obstet Gynecol 2004; 24(4):402-411.

54. Tonni B, De Felice C, Centini G, Gianneschi G. Cervical and oral teratome in the fetus: a systtematycreview of etiology, pathology, diagnosis, treatment and prognosis. Arch. Gynecol Obstet 2010.

55. Lee W, Kirk JS, Shaheen KW, Romero R, Hodges AN, Comstock $\mathrm{CH}$. Fetal cleft lip and palate detection by threedimensional ultrasonography. Ultrasound Obstet Gynecol 2000;16(4):299-301.

56. Hata T, Kenenishi K, Akiyama M, Tanaka H, Kimura K. Realtime 3-D sonographic observation of fetal facial expression. J Obstet Gynecol Res 2005;31(4):337-340.

57. Kurjak A, Stanojevic M, Azumendi G, Carrera JM. The potential of four-dimensional (4D) ultrasonography in the assessment of fetal awareness. J Perinat Med 2005;33(1):46-53

58. Yanagihara T, Hata T. Three-dimensional sonographic visualization of fetal skeleton in the second trimester of pregnancy. Gynecol Obstet Invest 2000;49(1):12-16.

59. Dikkeboom CM, Roelfsema NM, Van Adrichem LN, Wladimiroff JW. The role of three-dimensional ultrasound in visualizing the fetal cranial sutures and fontanels during the second half of pregnancy. Ultrasound Obstet Gynecol 2004; 24(4):412-416.

60. Faro C, Benoit B, Wegrzyn P, Chaoui R, Nicolaides KH. Three-dimensional sonographic description of the fetal frontal bones and metopic suture. Ultrasound Obstet Gynecol 2005;26(6):618-621.

61. Chaoui R, Levaillant JM, Benoit B, Faro C, Wegrzyn P, Nicolaides KH. Three-dimensional sonographic description of abnormal metopic suture in second-and third-trimester fetuses. Ultrasound Obstet Gynecol 2005;26(7):761-764.

62. Vinals F, Munoz M, Naveas R, Shalper J, Giuliano A. The fetal cerebellar vermis: anatomy and biometric assessment using 
volume contrast imaging in the C-plane(VCI-C). Ultrasound Obstet Gynecol 2005; 26(6):622-627.

63. Chang $\mathrm{CH}$, Chang FM, Yu CH, Ko HC, Chen HY. Assessment of fetal cerebellar volume using three dimensional ultrasound. Ultrasound Med Biol 2000;26(6):981-988.

64. Benacerraf, B. R., P. M. Sadow, C. E. Barnewolt, J. A. Estroff and C. Benson. Cleft of the secondary palate without cleft lip diagnosed with three-dimensional ultrasound and magnetic resonance imaging in a fetus with Fryns' syndrome. Ultrasound Obstet Gynecol 2006;27(5):566-570

65. Tonni G, Panteghini M, Pattacini P, De Felice C, Centini G, Ventura A. Integrating 3D sonography with targeted MRI in the prenatal diagnosis of posterior cleft lip. Journal of Diagnostic Medical Sonography 2006;22:367-371.

66. Tonni G, Centini G, Rosignoli L. Prenatal screening for fetal face and clefting in a prospective study on low-risk population: can 3-4 dimensional ultrasound enhance visualization and detection rate? Oral Pathology, Oral Radiology and Endodontics 2005;100:420-426.

67. Gorlin RJ, Cervenka J, Pruzansky S. Facial clefting and its syndromes. Birth Defects Orig Artic Ser 1971:7(7):3-49.

68. Calzolari E, Pierini A, Astolfi G, Bianchi F, Neville AJ,Rivieri F. Associated anomalies in multi-malformed infants with cleft lip and palate: An epidemiologic study of nearly 6 million births in 23 EUROCAT registries. Am J Med Genet A 2007;143(6):528-537.

69. Stoll C, Alembik Y, Dott B, Roth MP. Associated malformations in cases with oral clefts. Cleft Palate Craniofac J 2000;37(1): 41-47.

70. Calzolari E, Bianchi F, Rubini M, Ritvanen A, Neville AJ. Epidemiology of cleft palate in Europe: implications for genetic research. Cleft Palate Craniofac J 2004;41(3):244-249.

71. Milerad J, Larson O, Ph DD, Hagberg C, Ideberg M. Associated malformations in infants with cleft lip and palate: a prospective, population-based study. Pediatrics 1997:100(2 Pt 1):180-186.

72. Walker SJ, Ball RH, Babcook CJ, Feldkamp MM. Prevalence of aneuploidy and additional anatomic abnormalities in fetuses and neonates with cleft lip with or without cleft palate: a population-based study in Utah. J Ultrasound Med 2001,20(11):1175-1180.

73. Ghi T, Tani G, Savelli L, Colleoni GG, Pilu G, Bovicelli L. Prenatal imaging of facial clefts by magnetic resonance imaging with emphasis on the posterior palate. Prenat Diagn 2003;23(12):970-975.

74. Chmait R, Pretorius D, Moore T, Hull A, James G, Nelson T, Jones M. Prenatal detection of associated anomalies in fetuses diagnosed with cleft lip with or without cleft palate in utero. Ultrasound Obstet Gynecol 2006;27(2): 173-176.

75. Clementi M, Tenconi R, Bianchi F, Stoll C. Evaluation of prenatal diagnosis of cleft lip with or without cleft palate and cleft palate by ultrasound: experience from 20 European registries. EUROSCAN study group. Prenat Diagn 2000.20(11):870-875

76. Hanikeri M, Savundra J, Gillett D, Walters M,McBain W. Antenatal transabdominal ultrasound detection of cleft lip and palate in Western Australia from 1996 to 2003. Cleft Palate Craniofac J 2006;43(1): 61-66.

77. Walker SJ, Ball RH, Babcook CJ, Feldkamp MM. Prevalence of aneuploidy and additional anatomic abnormalities in fetuses and neonates with cleft lip with or without cleft palate. L Ultrasound Med 2001;20:1175-1180.
78. Mulliken JB, Benacerraf BR. Prenatal diagnosis of cleft lip: what the sonologist needs to tell the surgeon. J Ultrasound Med 2001:20(11): 1159-1164.

79. Berkowitz S. Cleft lip and palate: Diagnosis and management. Berlin, Springer-Verlag, 2006.

80. Sherer DM, Sokolovski M, Santoso PG, Dalloul M, Abulafia O. Nomograms of sonographic measurements throughout gestation of the fetal hard palate width, length and area. Ultrasound Obstet Gynecol 2004;24:35-41.

81. Shipp TD, Mulliken JB, Bromley B, Benacerraf B. Threedimensional prenatal diagnosis of frontonasal malformation and unilateral cleft lip/palate. Ultrasound Obstet Gynecol 2002;20:290-293.

82. Centini G, Rosignoli L, Faldini E, Voltolini C, Tonni G. Comparison between three different methods of scan to visualize the secondary foetal palate by thre-dimensional ultrasonography.17th World Congress on Ultrasound in Obstetrics and Gynecology. Florence. ISUOG 2007

83. Centini G, Rosignoli L, Faldini E. L'ecografia del primo e secondo trimestre in 3D.: 82-104. Vol. Diagnosi Prenatale Ed Paletto. 2006.

84. Campbell S, Lees C, Moscoso G, Hall P. Ultrasound antenatal diagnosis of cleft palate by a new technique: the 3D "reverse face" view. Ultrasound Obstet Gynecol 2000;25(1): 12-18.

85. Platt LD, Devore GR, Pretorius DH. Improving cleft palate/ cleft lip antenatal diagnosis by 3-dimensional sonography: the "flipped face" view. J Ultrasound Med 2006;25:1423-1430.

86. Faure JM, Captier G, Baumler M, Boulot P. Sonographic assessment of normal fetal palate using three-dimensional imaging: a new technique. Ultrasound Obstet Gynecol 2007;29(2): 159-165.

87. Pilu G, Segata M. A novel technique for visualization of the normal and cleft fetal secondary palate: angled insonation and three-dimensional ultrasound. Ultrasound Obstet Gynecol 2007;29(2): 166-169.

88. Tonni G, Centini G, Inaudi P, Rosignoli L, Ginanneschi C, De Felice C.Prenatal diagnosis of severe epignathus in a twin: case report and review of the literature. Cleft Palate Craniofac J 2010;47(4):421-425

89. Campbell S.Prenatal ultrasound examination of the secondary palate. Ultrasound Obstet Gynecol 2007, 29(2): 124-127.

90. Wilhelm L, Borgers $H$. The 'equals sign': a novel marker in the diagnosis of fetal isolated cleft palate. Ultrasound Obstet Gynecol 2010;36(4):439-444.

91. Cohen L, Mangers K, Grobman WA, Gotteiner N, Julien S, Dungan J, Fonseca L, Platt LD. Three-dimensional fast acquisition with sonographically based volume computeraided analysis for imaging of the fetal heart at 18 to 22 weeks' gestation. J Ultrasound Med 2010;29(5):751-757.

92. Ruano R, Martinovic J, Dommergues M, Aubry MC, Dumez $\mathrm{Y}$, Benachi A. Accuracy of fetal lung volume assessed by three-dimensional sonography. Ultrasound Obstet Gynecol 2005;26(7):725-730.

93. Ruano R, Benachi A, Joubin L, Aubry MC, Thalabard JC, Dumez Y, Dommergues M. Three dimensional ultrasonographic assessment of fetal lung volume as prognostic factor in isolated congenital diaphragmatic hernia. Brit J Ostet Gynaecol 2004;111(5):423-429.

94. Chang $\mathrm{CH}, \mathrm{Yu} \mathrm{CH}$, Chang FM, Ko HC, Chen HY. The assessment of normal fetal liver volume by three-dimensional ultrasound. Ultrasound Med Biol 2003;29(8):1123-1129. 
95. Rosignoli L, Periti E, Tonni G, Centini G, Tassinari M. VCIOmniview e studio della colonna vertebrale. XVII Congresso Nazionale Società Italiana Ecografia Ostetrica-Ginecologica (SIEOG)17-20 Ottobre Sorrento 2010.

96. Pilu GL,Ghi T. Preliminary experience with advanced volume contrast imaging (VCI) and OmniViewobetric and gynecologist ultrasound. GE Heltcare 2010 White book.

97. Lauson S, Alvarez C, Patel MS, Langlois S. Outcome of prenatally diagnosed isolated clubfoot. Ultrasound Obstet Gynecol 2010;35(6):708-714.

98. Cafici D, Iglesias A. Prenatal diagnosis of severe hypospadias with two-and three-dimensional sonography. J Ultrasound Med 2002;21(12):1423-1426.

99. Costa J,RiceH,Cardwell C, Hutte Ag, Ong S. An assessment of vascularity and flow intensity of the placenta in normal pregnancy and pre-eclampsia using three-dimensional ultrasound. J MaternFetal Neonatal Med 2010;23(8):894-899.
100. Huster KM, Haas K, Schoenborn J, McVean D, Odibo AO.Reproducibility of placental volume and vasculature indices obtained by 3-dimensional power Doppler sonography. J Ultrasound Med 2010;29(6):911-916.

101. D'Elia A, Pighetti M, Moccia G, Santangelo N. Spontaneous motor activity in normal fetuses. Early Hum Dev 2001;65(2):139-147.

102. Robles de Medina PG, Visser GH, Huizink AC, Buitelaar JK, Mulder EJ. Fetal behaviour does not differ between boys and girls. Early Hum Dev 2003;73(1-2):17-26.

103. Kuno A, Akiyama M, Yamashiro C, Tanaka H, Yanagihara $\mathrm{T}$, Hata T. Three-dimensional sonographic assessment of fetal behaviour in the early second trimester of pregnancy. J Ultrasound Med 2001; 20(12):1271-1275.

104. Blaas HG, Eik-Nes SH. Sonoembryology and early prenatal diagnosis of neural anomalies. Prenat Diagn 2009;29(4): 312-325. 